\title{
Supplementary materials for: Influence of Polarisability on the Prediction of Electrical Double Layer Structure in a Clay Mesopore: A Molecular Dynamics Study
}

Sébastien Le Crom ${ }^{1,2}$, Christophe Tournassat ${ }^{3,4,5}$, Jean-Charles Robinet ${ }^{2}$ and Virginie Marry ${ }^{1}$

${ }^{1}$ Sorbonne Université, CNRS,

PHysico-chimie des Électrolytes et Nano-systèmes InterfaciauX, PHENIX, 4 place Jussieu, F-75005, Paris, France

${ }^{2}$ Agence Nationale pour la Gestion des Déchets Radioactifs (ANDRA), Parc de la Croix Blanche rue Jean Monnet, F-92298, Chatenay Malabry, France

${ }^{3}$ Bureau de recherches géologiques et minières (BRGM), 3 Avenue Claude Guillemin, F-45100 Orléans, France

${ }^{4}$ UMR 7327 Institut des Sciences de la Terre d'Orléans, Université d'Orléans-CNRS/INSU-BRGM, F-45071 Orléans, France

${ }^{5}$ Earth and Environmental Sciences Area, Energy Geosciences Division, Lawrence Berkeley National Laboratory, Berkeley, CA 94720, USA 


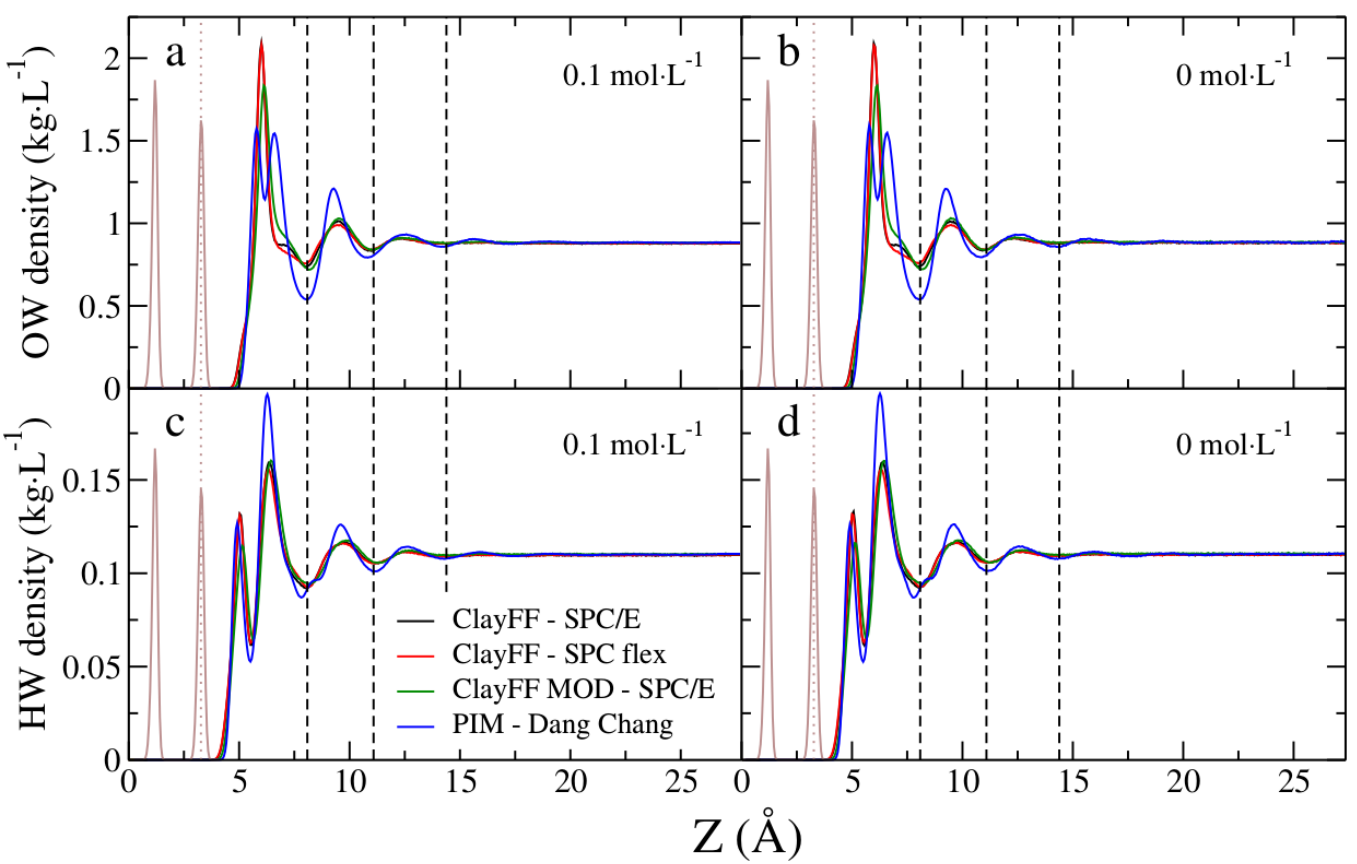

Figure S1: Density distribution profiles in the direction normal to the clay surface of (a) water oxygen atoms in the presence of added $0.1 \mathrm{~mol} \cdot \mathrm{L}^{-1} \mathrm{NaCl}$. (b) water oxygen atoms in the absence of added $0.1 \mathrm{~mol} \cdot \mathrm{L}^{-1} \mathrm{NaCl}$. (c) water hydrogen atoms in the presence of added $0.1 \mathrm{~mol} \cdot \mathrm{L}^{-1} \mathrm{NaCl}(\mathrm{d})$ water hydrogen atoms in the absence of added $0.1 \mathrm{~mol} \cdot \mathrm{L}^{-1} \mathrm{NaCl}$. The coordinate $\mathrm{z}$ $=0 \AA$ corresponds to the center of the clay layer. The clay oxygen distributions are shown in brown and the center of mass of the surface oxygen is represented by a brown dotted line at $z=3.27 \AA$. The surface water layers are delimited by the dashed black lines. 


\section{\# input LAMMPS for ClayFF-SPCE}

units real

dimension 3

boundary $\mathrm{p} \mathrm{p} \mathrm{p}$

atom_style full

bond_style harmonic

angle_style harmonic

read_data_topology_file-ClayFF-SPCE.dat

\# 1=OW, $2=\mathrm{HW}, 3=\mathrm{Al}, 4=\mathrm{Si}, 5,6,7=\mathrm{O}, 8=\mathrm{H}, 9=\mathrm{Mg}, 10=\mathrm{Na}, 11=\mathrm{Cl}$

group oxy type 1

group water type 12

group clay type 3456789

group Na type 10

group $\quad \mathrm{Cl}$ type 11

group mobile type 121011

pair_style lj/cut/coul/long 10.

\#Lennard-Jones

pair_coeff $\quad 110.155400000003 .16550010 .000000$

pair_coeff 220.000000000000 .00000010 .000000

pair_coeff 330.000001329804 .27120010 .000000

pair_coeff 440.000001840503 .30203010 .000000

pair_coeff 550.155400000003 .16550010 .000000

pair_coeff $\quad 660.155400000003 .16550010 .000000$

pair_coeff 770.155400000003 .16550010 .000000

pair_coeff $\quad 880.000000000000 .00000010 .000000$

pair_coeff 990.000000902985 .26430010 .000000

pair_coeff 10100.130100000002 .35000010 .000000

pair_coeff 11110.100100000004 .39990010 .000000

pair_modify mix arithmetic

\#Bond Types

bond_coeff $\quad 1554.13491 .0$

bond_coeff 2554.13491 .0

\#Angle Types

angle_coeff $\quad 145.7696109 .47$

angle_coeff $230.0 \quad 109.47$

kspace_style pppm 0.0001

dielectric 1.0

neighbor 2.0 bin

neigh_modify delay 0 check yes every 1 \#one 3000 


$\begin{array}{ll}\begin{array}{ll}\text { timestep } \\ \text { run_style }\end{array} & \text { verlet } \\ & \\ \text { compute } & \quad \text { watertemp water temp } \\ \text { compute } & \text { claytemp clay temp } \\ \text { compute } & \text { mytemp all temp } \\ \text { compute } & \text { mobtemp mobile temp }\end{array}$

fix $\quad 2$ water shake $0.0001100 \mathrm{~b} 1$ a 1

fix $\quad 1$ all nvt temp 3003001000.0

fix_modify 1 temp mytemp

fix $\quad 3$ all momentum 1 linear 111

thermo_style custom step c_mytemp c_watertemp c_claytemp ke pe etotal press evdwl ecoul elong lz thermo 100

restart $\quad 1000000$ restart.*.mesopore_clayff-spce

dump $\quad 1$ all atom 1000 trajectory.lammpstrj

dump_modify 1 sort id

run 20000000 
\# input CP2K for PIM-Dang Chang

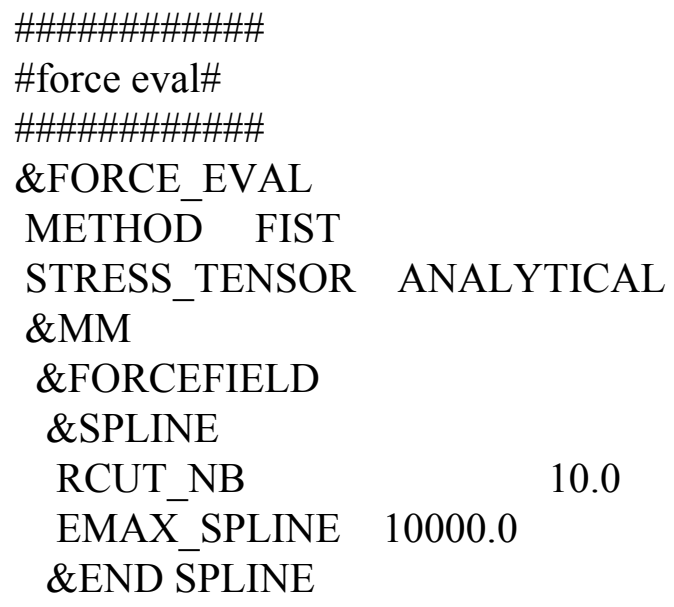

R0

0.33070207325498757

\&END BOND 


\section{KIND}

K

R0

\&END BOND

$\&$ BOND

ATOMS

KIND

K

R0

\&END BOND

\#\#\#\#\#

\#BEND\#

\#\#\#\#\#

\&BEND

ATOMS

KIND

K

THETA0

\&END BEND

\&BEND

ATOMS

KIND

K

THETA0

\&END BEND

\&BEND

ATOMS

KIND

K

THETA0

\&END BEND

\&BEND

ATOMS

KIND

K

THETA0

\&END BEND

\&BEND

ATOMS

KIND

K

THETA0

\&END BEND

\&BEND

ATOMS

KIND

K

THETA0

\&END BEND

\&BEND
G87

0.00000000000000000

0.33070207325498757

HW MW

G87

0.00000000000000000

0.33070207325498757
HW OW HW

G87

0.00000000000000000

1.91113553093379100

HW OW MW

G87

0.00000000000000000

0.95567765466895480

MW HW OW

G87

0.00000000000000000

1.91113553093379100

MW HW HW

G87

0.00000000000000000

1.91113553093379100

OW HW HW

G87

0.00000000000000000

1.91113553093379100

OW MW HW

G87

0.00000000000000000

1.91113553093379100 


\section{ATOMS}

KIND

$\mathrm{K}$

THETA0

\&END BEND

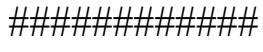

\#NON BONDED\#

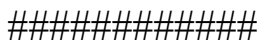

\section{\&NONBONDED}

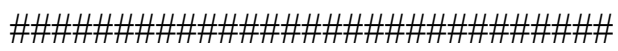

\# LENNARD-JONES CLAY ATOMS \#

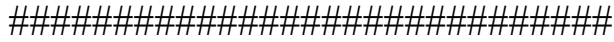

\begin{tabular}{|c|c|}
\hline \multicolumn{2}{|c|}{ \&LENNARD-JONES } \\
\hline ATOMS & $\mathrm{OH}$ \\
\hline EPSILON & $0.0000000000000000 \mathrm{E}+00$ \\
\hline SIGMA & $1.0000000000000001 \mathrm{E}-01$ \\
\hline \multicolumn{2}{|c|}{ RCUT 4.2000} \\
\hline \multicolumn{2}{|c|}{ \&END LENNARD-JONES } \\
\hline \multicolumn{2}{|c|}{ \&LENNARD-JONES } \\
\hline ATOMS & $\mathrm{H} \mathrm{H}$ \\
\hline EPSILON & $0.0000000000000000 \mathrm{E}+00$ \\
\hline SIGMA & $1.0000000000000001 \mathrm{E}-01$ \\
\hline RCUT & 4.2000 \\
\hline \multicolumn{2}{|c|}{ \&END LENNARD-JONES } \\
\hline \multicolumn{2}{|c|}{ \&LENNARD-JONES } \\
\hline ATOMS & $\mathrm{OH} \mathrm{H}$ \\
\hline EPSILON & $0.0000000000000000 \mathrm{E}+00$ \\
\hline SIGMA & $1.0000000000000001 \mathrm{E}-01$ \\
\hline RCUT & 4.2000 \\
\hline \multicolumn{2}{|c|}{ \&END LENNARD-JONES } \\
\hline \multicolumn{2}{|c|}{ \&LENNARD-JONES } \\
\hline ATOMS & Si H \\
\hline EPSILON & $0.0000000000000000 \mathrm{E}+00$ \\
\hline SIGMA & $1.0000000000000001 \mathrm{E}-01$ \\
\hline RCUT & 4.2000 \\
\hline \multicolumn{2}{|c|}{ \&END LENNARD-JONES } \\
\hline \multicolumn{2}{|c|}{ \&LENNARD-JONES } \\
\hline ATOMS & $\mathrm{Al} \mathrm{H}$ \\
\hline EPSILON & $0.0000000000000000 \mathrm{E}+00$ \\
\hline SIGMA & $1.0000000000000001 \mathrm{E}-01$ \\
\hline RCUT & 4.2000 \\
\hline \multicolumn{2}{|c|}{ \&END LENNARD-JONES } \\
\hline \multicolumn{2}{|c|}{ \&LENNARD-JONES } \\
\hline ATOMS & $\mathrm{MgA} \mathrm{H}$ \\
\hline EPSILON & $0.0000000000000000 \mathrm{E}+00$ \\
\hline SIGMA & $1.0000000000000001 \mathrm{E}-01$ \\
\hline RCUT & 4.2000 \\
\hline \&END LEN & INARD-JONES \\
\hline
\end{tabular}

HW MW HW

G87

1.91113553093379100 
\&LENNARD-JONES

$\begin{array}{ll}\text { ATOMS } & \text { Si Si } \\ \text { EPSILON } & 0.0000000000000000 \mathrm{E}+00 \\ \text { SIGMA } & 1.0000000000000001 \mathrm{E}-01 \\ \text { RCUT } & 4.2000 \\ \text { \&END LENNARD-JONES } \\ \text { \&LENNARD-JONES } \\ \text { ATOMS } & \text { Si Al } \\ \text { EPSILON } & 0.0000000000000000 \mathrm{E}+00 \\ \text { SIGMA } & 1.0000000000000001 \mathrm{E}-01 \\ \text { RCUT } & 4.2000\end{array}$

\&END LENNARD-JONES

\&LENNARD-JONES

$\begin{array}{ll}\text { ATOMS } & \text { Si MgA } \\ \text { EPSILON } & 0.0000000000000000 \mathrm{E}+00 \\ \text { SIGMA } & 1.0000000000000001 \mathrm{E}-01 \\ \text { RCUT } & 4.2000\end{array}$

\&END LENNARD-JONES

\&LENNARD-JONES

$\begin{array}{lc}\text { ATOMS } & \text { Al Al } \\ \text { EPSILON } & 0.0000000000000000 \mathrm{E}+00 \\ \text { SIGMA } & 1.0000000000000001 \mathrm{E}-01 \\ \text { RCUT } & 4.2000 \\ \text { \&END LENNARD-JONES } & \\ \text { \&LENNARD-JONES } \\ \text { ATOMS } & \text { MgA Al } \\ \text { EPSILON } & 0.0000000000000000 \mathrm{E}+00 \\ \text { SIGMA } & 1.0000000000000001 \mathrm{E}-01 \\ \text { RCUT } & 4.2000 \\ \text { \&END LENNARD-JONES } \\ \text { \&LENNARD-JONES } \\ \text { ATOMS } \\ \text { EPSILON } & \text { MgA MgA } \\ \text { SIGMA } & 0.0000000000000000 \mathrm{E}+00 \\ \text { RCUT } & 1.0000000000000001 \mathrm{E}-01 \\ \text { \&END LENNARD-JONES }\end{array}$

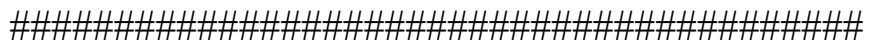

\# LJ WATER \#

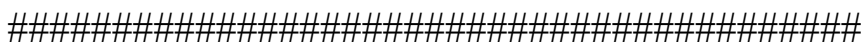

$\begin{array}{lc}\text { \&LENNARD-JONES } & \\ \text { ATOMS } & \text { OW OW } \\ \text { EPSILON } & 9.1837456031403335 \mathrm{E}+01 \\ \text { SIGMA } & 3.2340000000000009 \mathrm{E}+00 \\ \text { RCUT } & 1.0000000000000000 \mathrm{E}+01 \\ \text { \&END LENNARD-JONES } \\ \text { \&LENNARD-JONES } \\ \text { ATOMS } \\ \text { EPSILON } & \text { OW MW } \\ \text { SIGMA } & 0.0000000000000000 \mathrm{E}+00 \\ \text { \&END LENNARD-JONES }\end{array}$


\&LENNARD-JONES

$\begin{array}{ll}\text { ATOMS } & \text { OW HW } \\ \text { EPSILON } & 0.0000000000000000 \mathrm{E}+00 \\ \text { SIGMA } & 1.0000000000000001 \mathrm{E}-01 \\ \text { \&END LENNARD-JONES } & \\ \text { \&LENNARD-JONES } & \\ \text { ATOMS } & \text { HW HW } \\ \text { EPSILON } & 0.0000000000000000 \mathrm{E}+00 \\ \text { SIGMA } & 1.0000000000000001 \mathrm{E}-01 \\ \text { \&END LENNARD-JONES } & \\ \text { \&LENNARD-JONES } \\ \text { ATOMS } \\ \text { EPSILON } & \text { HW MW } \\ \text { SIGMA } & 0.0000000000000000 \mathrm{E}+00 \\ \text { \&END LENNARD-JONES } & 1.0000000000000001 \mathrm{E}-01 \\ \text { \&LENNARD-JONES } & \\ \text { ATOMS } & \text { MW MW } \\ \text { EPSILON } & 0.0000000000000000 \mathrm{E}+00 \\ \text { SIGMA } & 1.0000000000000001 \mathrm{E}-01 \\ \text { \&END LENNARD-JONES }\end{array}$

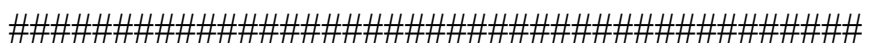

\# LJ between CLAY ATOMS and WATER \#

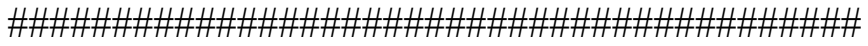

\section{\&LENNARD-JONES}

$\begin{array}{ll}\text { ATOMS } & \text { Al HW } \\ \text { EPSILON } & 0.0000000000000000 \mathrm{E}+00 \\ \text { SIGMA } & 1.0000000000000001 \mathrm{E}-01 \\ \text { \&END LENNARD-JONES } & \\ \text { \&LENNARD-JONES } & \text { MgA HW } \\ \text { ATOMS } & 0.0000000000000000 \mathrm{E}+00 \\ \text { EPSILON } & 1.0000000000000001 \mathrm{E}-01 \\ \text { SIGMA } & \text { Al MW } \\ \text { \&END LENNARD-JONES } \\ \text { \&LENNARD-JONES } \\ \text { ATOMS } & 0.0000000000000000 \mathrm{E}+00 \\ \text { EPSILON } & 1.0000000000000001 \mathrm{E}-01 \\ \text { SIGMA } & \\ \text { \&END LENNARD-JONES } & \\ \text { \&LENNARD-JONES } & \\ \text { ATOMS } & \text { MgA MW } \\ \text { EPSILON } & 0.0000000000000000 \mathrm{E}+00 \\ \text { SIGMA } & 1.0000000000000001 \mathrm{E}-01 \\ \text { \&END LENNARD-JONES } & \\ \text { \&LENNARD-JONES } & \\ \text { ATOMS } & \text { Si HW } \\ \text { EPSILON } & 0.0000000000000000 \mathrm{E}+00 \\ \text { SIGMA } & 1.0000000000000001 \mathrm{E}-01 \\ \text { \&END LENNARD-JONES } \\ \text { \&LENNARD-JONES } \\ \text { ATOMS } & \text { Si MW } \\ & \end{array}$




\begin{tabular}{|c|c|}
\hline EPSILON & $0.0000000000000000 \mathrm{E}+00$ \\
\hline SIGMA & $1.0000000000000001 \mathrm{E}-01$ \\
\hline \multicolumn{2}{|c|}{ \&END LENNARD-JONES } \\
\hline \multicolumn{2}{|c|}{ \&LENNARD-JONES } \\
\hline ATOMS & O HW \\
\hline EPSILON & $0.0000000000000000 \mathrm{E}+00$ \\
\hline SIGMA & $1.0000000000000001 \mathrm{E}-01$ \\
\hline \multicolumn{2}{|c|}{ \&END LENNARD-JONES } \\
\hline \multicolumn{2}{|c|}{ \&LENNARD-JONES } \\
\hline ATOMS & $\mathrm{O}$ MW \\
\hline EPSILON & $0.0000000000000000 \mathrm{E}+00$ \\
\hline SIGMA & $1.0000000000000001 \mathrm{E}-01$ \\
\hline \multicolumn{2}{|c|}{ \&END LENNARD-JONES } \\
\hline \multicolumn{2}{|c|}{ \&LENNARD-JONES } \\
\hline ATOMS & OH HW \\
\hline EPSILON & 0.000000000 \\
\hline SIGMA & $000000001 \mathrm{E}-01$ \\
\hline \multicolumn{2}{|c|}{ \&END LENNARD-JONES } \\
\hline \multicolumn{2}{|c|}{ \&LENNARD-JONES } \\
\hline ATOMS & $\mathrm{OH}$ MW \\
\hline EPSILON & $0000 \mathrm{E}+00$ \\
\hline SIGMA & 1.0000( \\
\hline \multicolumn{2}{|c|}{ \&END LENNARD-JONES } \\
\hline \multicolumn{2}{|c|}{ \&LENNARD-JONES } \\
\hline ATOMS & H OW \\
\hline EPSILON & $0.0000000000000000 \mathrm{E}+00$ \\
\hline SIGMA & $1.0000000000000001 \mathrm{E}-01$ \\
\hline \multicolumn{2}{|c|}{ \&END LENNARD-JONES } \\
\hline \multicolumn{2}{|c|}{ \&LENNARD-JONES } \\
\hline ATOMS & H HW \\
\hline EPSILON & 0.00000 \\
\hline SIGMA & $1.0000000000000001 \mathrm{E}-01$ \\
\hline \multicolumn{2}{|c|}{ \&END LENNARD-JONES } \\
\hline \multicolumn{2}{|c|}{ \&LENNARD-JONES } \\
\hline ATOMS & H MW \\
\hline EPSILON & $0.0000000000000000 \mathrm{E}+00$ \\
\hline SIGMA & $1.0000000000000001 \mathrm{E}-01$ \\
\hline \&END LF & ONES \\
\hline
\end{tabular}

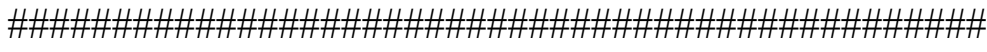

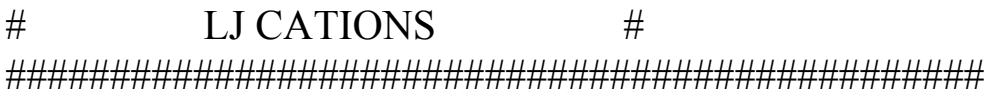

\&LENNARD-JONES

ATOMS $\quad \mathrm{Na} \mathrm{Na}$

EPSILON $\quad 0.0000000000000000 \mathrm{E}+00$

SIGMA $\quad 1.0000000000000001 \mathrm{E}-01$

\&END LENNARD-JONES 
\&LENNARD-JONES

ATOMS

$\mathrm{Cl} \mathrm{Cl}$

EPSILON

$0.0000000000000000 \mathrm{E}+00$

SIGMA

$1.0000000000000001 \mathrm{E}-01$

\&END LENNARD-JONES

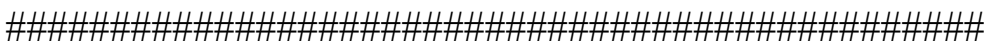

\# LJ between CATIONS and CLAY ATOMS \#

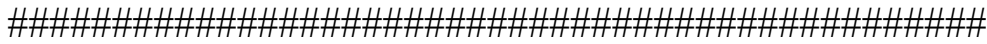

\&LENNARD-JONES

ATOMS

$\mathrm{Na} \mathrm{Si}$

EPSILON

$0.0000000000000000 \mathrm{E}+00$

SIGMA

$1.0000000000000001 \mathrm{E}-01$

\&END LENNARD-JONES

\&LENNARD-JONES

ATOMS

$\mathrm{Na} \mathrm{Al}$

EPSILON

$0.0000000000000000 \mathrm{E}+00$

SIGMA

$1.0000000000000001 \mathrm{E}-01$

\&END LENNARD-JONES

\&LENNARD-JONES

ATOMS

$\mathrm{Na} \mathrm{MgA}$

EPSILON

$0.0000000000000000 \mathrm{E}+00$

SIGMA

$1.0000000000000001 \mathrm{E}-01$

\&END LENNARD-JONES

\&LENNARD-JONES

ATOMS

$\mathrm{NaH}$

EPSILON

$0.0000000000000000 \mathrm{E}+00$

SIGMA

$1.0000000000000001 \mathrm{E}-01$

\&END LENNARD-JONES

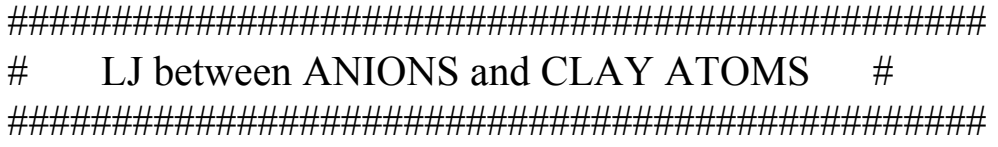

\&LENNARD-JONES

ATOMS $\quad \mathrm{Cl} \mathrm{Si}$

EPSILON $\quad 0.0000000000000000 \mathrm{E}+00$

SIGMA $\quad 1.0000000000000001 \mathrm{E}-01$

\&END LENNARD-JONES

\&LENNARD-JONES

ATOMS

$\mathrm{Cl} \mathrm{Al}$

EPSILON

$0.0000000000000000 \mathrm{E}+00$

SIGMA

$1.0000000000000001 \mathrm{E}-01$

\&END LENNARD-JONES

\&LENNARD-JONES

ATOMS

$\mathrm{Cl} \mathrm{MgA}$

EPSILON

$0.0000000000000000 \mathrm{E}+00$

SIGMA

$1.0000000000000001 \mathrm{E}-01$

\&END LENNARD-JONES 
\&LENNARD-JONES

ATOMS

$\mathrm{Cl} \mathrm{H}$

EPSILON

$0.0000000000000000 \mathrm{E}+00$

SIGMA

$1.0000000000000001 \mathrm{E}-01$

\&END LENNARD-JONES

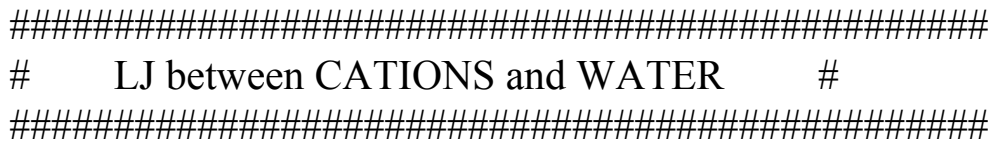

\&LENNARD-JONES

ATOMS

$\mathrm{Na}$ MW

EPSILON

$0.0000000000000000 \mathrm{E}+00$

SIGMA

$1.0000000000000001 \mathrm{E}-01$

\&END LENNARD-JONES

\&LENNARD-JONES

ATOMS

$\mathrm{Na}$ HW

EPSILON

$0.0000000000000000 \mathrm{E}+00$

SIGMA

$1.0000000000000001 \mathrm{E}-01$

\&END LENNARD-JONES

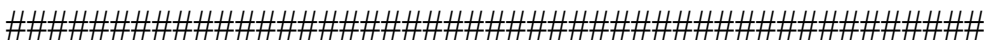

\# LJ between ANIONS and WATER \#

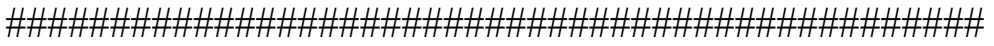

\&LENNARD-JONES
ATOMS
$\mathrm{Cl} \mathrm{MW}$
EPSILON
$0.0000000000000000 \mathrm{E}+00$
SIGMA
$1.0000000000000001 \mathrm{E}-01$

\&END LENNARD-JONES

\&LENNARD-JONES

ATOMS

$\mathrm{Cl} \mathrm{HW}$

EPSILON

$0.0000000000000000 \mathrm{E}+00$

SIGMA

$1.0000000000000001 \mathrm{E}-01$

\&END LENNARD-JONES

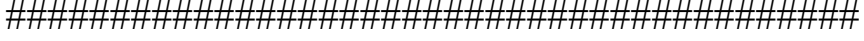 \\ \# BMHFTD CLAY ATOMS \#

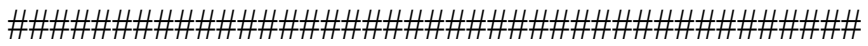

\section{\&BMHFTD}

ATOMS

A

B

C

$\mathrm{D}$

$\mathrm{BD}$

RCUT

\&END BMHFTD

\&BMHFTD

ATOMS

$\mathrm{O} \mathrm{Al}$

A

$6.0001294194 \mathrm{E}+01$
$\mathrm{OO}$

$2.8374878334 \mathrm{E}+01$

$5.4859546247 \mathrm{E}+00$

1.3258

7.3458

2.718

$1.0000000 \mathrm{E}+01$ 


\begin{tabular}{|c|c|}
\hline B & $3.3261768412 \mathrm{E}+00$ \\
\hline $\mathrm{C}$ & 0.047736 \\
\hline $\mathrm{D}$ & 0.1556 \\
\hline BD & 4.1682 \\
\hline RCUT & $1.0000000 \mathrm{E}+01$ \\
\hline \multicolumn{2}{|c|}{ \&END BMHFTD } \\
\hline \multicolumn{2}{|c|}{ \&BMHFTD } \\
\hline ATOMS & $\mathrm{O} \mathrm{MgA}$ \\
\hline A & $8.6276730471 \mathrm{E}+01$ \\
\hline $\mathrm{B}$ & $3.6607030016 \mathrm{E}+00$ \\
\hline $\mathrm{C}$ & 0.047736 \\
\hline $\mathrm{D}$ & 0.1556 \\
\hline BD & 4.1682 \\
\hline RCUT & $1.0000000 \mathrm{E}+01$ \\
\hline \multicolumn{2}{|c|}{ \&END BMHFTD } \\
\hline \multicolumn{2}{|c|}{ \&BMHFTD } \\
\hline ATOMS & $\mathrm{O} \mathrm{Si}$ \\
\hline A & $2.9401773508 \mathrm{E}+01$ \\
\hline $\mathrm{B}$ & $2.8348330546 \mathrm{E}+00$ \\
\hline $\mathrm{C}$ & 0.047736 \\
\hline $\mathrm{D}$ & 0.1556 \\
\hline BD & 4.1682 \\
\hline RCUT & $1.0000000 \mathrm{E}+01$ \\
\hline \multicolumn{2}{|c|}{ \&END BMHFTD } \\
\hline \multicolumn{2}{|c|}{ \&BMHFTD } \\
\hline ATOMS & $\mathrm{O} \mathrm{OH}$ \\
\hline A & $9.9000089400 \mathrm{E}+01$ \\
\hline B & $3.6000000000 \mathrm{E}+00$ \\
\hline $\mathrm{C}$ & 0.974 \\
\hline $\mathrm{D}$ & 5.247 \\
\hline $\mathrm{BD}$ & 2.718 \\
\hline RCUT & $1.0000000 \mathrm{E}+01$ \\
\hline \multicolumn{2}{|c|}{ \&END BMHFTD } \\
\hline \multicolumn{2}{|c|}{ \&BMHFTD } \\
\hline ATOMS & $\mathrm{OH} \mathrm{Si}$ \\
\hline A & $9.7980177079 \mathrm{E}+02$ \\
\hline B & $4.8247711081 \mathrm{E}+00$ \\
\hline $\mathrm{C}$ & 0.047736 \\
\hline $\mathrm{D}$ & 0.1556 \\
\hline BD & 4.1682 \\
\hline RCUT & $1.0000000 \mathrm{E}+01$ \\
\hline \multicolumn{2}{|c|}{ \&END BMHFTD } \\
\hline \multicolumn{2}{|c|}{ \&BMHFTD } \\
\hline ATOMS & $\mathrm{OH} \mathrm{Al}$ \\
\hline A & $2.0271645812 \mathrm{E}+01$ \\
\hline B & $3.1462603516 \mathrm{E}+00$ \\
\hline $\mathrm{C}$ & 0.047736 \\
\hline $\mathrm{D}$ & 0.1556 \\
\hline BD & 4.1682 \\
\hline RCUT & $1.0000000 \mathrm{E}+01$ \\
\hline \multicolumn{2}{|c|}{ \&END BMHFTI } \\
\hline & \\
\hline
\end{tabular}




\begin{tabular}{ll} 
ATOMS & \multicolumn{1}{c}{ OH MgA } \\
A & $3.5622652615 \mathrm{E}+01$ \\
B & $3.4854309519 \mathrm{E}+00$ \\
C & 0.047736 \\
D & 0.1556 \\
BD & 4.1682 \\
RCUT & $1.0000000 \mathrm{E}+01$ \\
\&END BMHFTD & \\
\&BMHFTD & \\
ATOMS & OH OH \\
A & $7.9476607457 \mathrm{E}+01$ \\
B & $3.2998379155 \mathrm{E}+00$ \\
C & 0.974 \\
D & 5.247 \\
BD & 2.718 \\
RCUT & $1.0000000 \mathrm{E}+01$ \\
\&END BMHFTD &
\end{tabular}

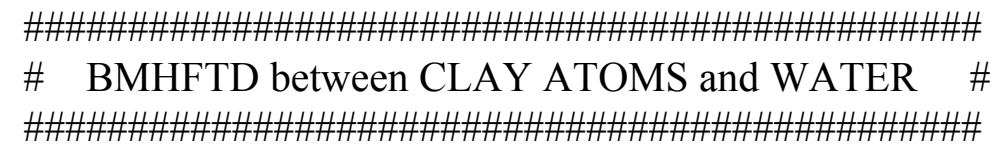

\&BMHFTD

\begin{tabular}{ll} 
ATOMS & \multicolumn{1}{c}{$\mathrm{Al} \mathrm{OW}$} \\
A & $1.1156055642 \mathrm{E}+02$ \\
B & $4.2315612261 \mathrm{E}+00$ \\
C & 0.047736 \\
D & 0.1556 \\
BD & 4.1682 \\
RCUT & $1.0000000 \mathrm{E}+01$
\end{tabular}

\&END BMHFTD

\&BMHFTD

\begin{tabular}{ll} 
ATOMS & \multicolumn{1}{c}{ MgA OW } \\
A & $1.5019041377 \mathrm{E}+02$ \\
B & $3.1699974072 \mathrm{E}+00$ \\
C & 0.047736 \\
D & 0.1556 \\
BD & 4.1682 \\
RCUT & $1.0000000 \mathrm{E}+01$
\end{tabular}

\&END BMHFTD

\&BMHFTD

\begin{tabular}{ll} 
ATOMS & \multicolumn{1}{c}{$\mathrm{Si} \mathrm{OW}$} \\
A & $7.2061011621 \mathrm{E}+01$ \\
B & $5.6198620615 \mathrm{E}+00$ \\
C & 0.047736 \\
D & 0.1556 \\
BD & 4.1682 \\
RCUT & $1.0000000 \mathrm{E}+01$ \\
\&END BMHFTD &
\end{tabular}

\&END BMHFTD

\&BMHFTD

$\begin{array}{lc}\text { ATOMS } & \text { O OW } \\ \text { A } & 3.6001004195 \mathrm{E}+02\end{array}$




$\begin{array}{ll}\text { B } & 3.7624572104 \mathrm{E}+00 \\ \text { C } & 0.974 \\ \text { D } & 5.247 \\ \text { BD } & 2.718 \\ \text { RCUT } & 1.0000000 \mathrm{E}+01 \\ \text { \&END BMHFTD } & \\ \text { \&BMHFTD } & \\ \text { ATOMS } & \text { OH OW } \\ \text { A } & 1.0102032807 \mathrm{E}+02 \\ \text { B } & 4.3399993000 \mathrm{E}+00 \\ \text { C } & 0.974 \\ \text { D } & 5.247 \\ \text { BD } & 2.718 \\ \text { RCUT } & 1.0000000 \mathrm{E}+01 \\ \text { \&END BMHFTD } & \end{array}$

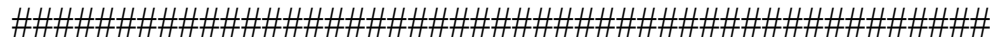
\# BMHFTD between CATIONS and CLAY ATOMS \#

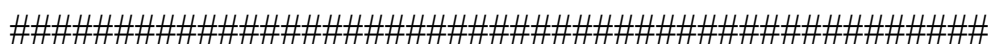

$\begin{array}{ll}\text { \&BMHFTD } & \\ \text { ATOMS } & \mathrm{NaO} \\ \text { A } & 3.0002137393 \mathrm{E}+01 \\ \text { B } & 3.2995018775 \mathrm{E}+00 \\ \text { C } & 0.047736 \\ \text { D } & 0.1556 \\ \text { BD } & 4.1682 \\ \text { RCUT } & 1.0000000 \mathrm{E}+01 \\ \text { \&END BMHFTD } & \\ \text { \&BMHFTD } & \\ \text { ATOMS } & \mathrm{Na} \mathrm{OH} \\ \text { A } & 4.0191122956 \mathrm{E}+03 \\ \text { B } & 5.9984492492 \mathrm{E}+00 \\ \text { C } & 0.047736 \\ \text { D } & 0.1556 \\ \text { BD } & 4.1682 \\ \text { RCUT } & 1.0000000 \mathrm{E}+01 \\ \text { \&END BMHFTD } & \end{array}$

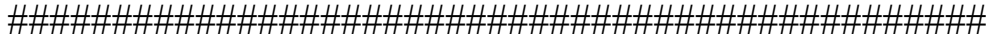
\# BMHFTD between ANIONS and CLAY ATOMS \#

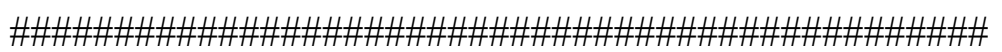

$\begin{array}{lc}\text { \&BMHFT } & \\ \text { ATOMS } & \mathrm{ClO} \\ \text { A } & 4.9960000000 \mathrm{E}+02 \\ \text { B } & 3.5600000000 \mathrm{E}+00 \\ \text { C } & 2.0390 \\ \text { D } & 4.2960 \\ \text { RCUT } & 1.0000000 \mathrm{E}+01 \\ \text { \&END BMHFT } & \\ \text { \&BMHFT } & \end{array}$




\begin{tabular}{ll} 
ATOMS & \multicolumn{1}{c}{$\mathrm{ClOH}$} \\
A & $4.9960000000 \mathrm{E}+02$ \\
B & $3.5600000000 \mathrm{E}+00$ \\
C & 2.0390 \\
D & 4.2960 \\
RCUT & $1.0000000 \mathrm{E}+01$ \\
\&END BMHFT &
\end{tabular}

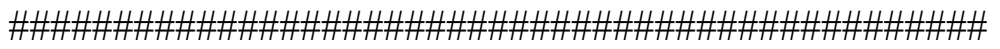

\# BMHFTD between CATIONS and WATER \#

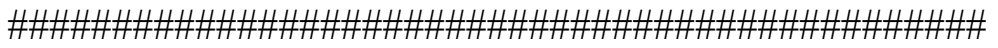

\section{\&BMHFTD}

$\begin{array}{lc}\text { ATOMS } & \mathrm{Na} \mathrm{OW} \\ \text { A } & 7.1108000000000004 \mathrm{E}+02 \\ \text { B } & 5.0613999999999999 \mathrm{E}+00 \\ \text { C } & 1.3350000000000001 \mathrm{E}-01 \\ \text { D } & 1.572600000000001 \mathrm{E}-01 \\ \text { BD } & 3.0000000000000000 \mathrm{E}+00 \\ \text { RCUT } & 1.0000000000000000 \mathrm{E}+01 \\ \text { \&END BMHFTD } & \end{array}$

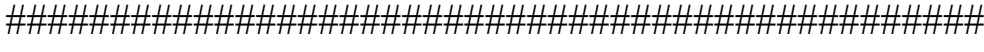

\# BMHFTD between ANIONS and WATER \#

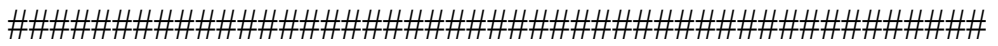

\&BMHFT
ATOMS
$\mathrm{Cl} \mathrm{OW}$

A

$4.9960000000000002 \mathrm{E}+02$

B $\quad 3.5600000000000001 \mathrm{E}+00$

C $\quad 2.0390000000000001 \mathrm{E}+00$

$\mathrm{D} \quad 4.2960000000000003 \mathrm{E}+00$

RCUT $\quad 1.0000000000000000 \mathrm{E}+01$

\&END BMHFT

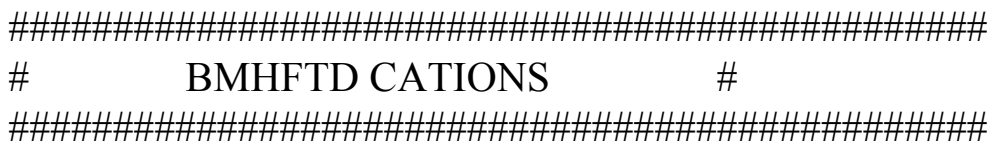

\section{\&BMHFTD}
ATOMS
$\mathrm{Na} \mathrm{Na}$
A
$1.7010000000000000 \mathrm{E}-02$
B $\quad 4.9650000000000000 \mathrm{E}+00$
C $2.9140000000000000 \mathrm{E}-02$
D $\quad 1.3940000000000000 \mathrm{E}-02$
BD $\quad 4.9650000000000000 \mathrm{E}+00$
RCUT
$1.0000000000000000 \mathrm{E}+01$
\&END BMHFTD 


$\begin{array}{lc}\text { \&BMHFTD } & \\ \text { ATOMS } & \mathrm{Cl} \mathrm{Cl} \\ \text { A } & 6.9840000000000000 \mathrm{E}+02 \\ \text { B } & 3.7770000000000000 \mathrm{E}+00 \\ \text { C } & 5.9510000000000000 \mathrm{E}+00 \\ \text { D } & 1.2850000000000000 \mathrm{E}+01 \\ \text { BD } & 1.6500000000000000 \mathrm{E}+00 \\ \text { RCUT } & 1.0000000000000000 \mathrm{E}+01 \\ \text { \&END BMHFTD } & \end{array}$

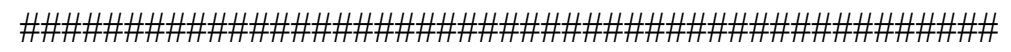

\# BMHFTD between CATIONS and ANIONS \#

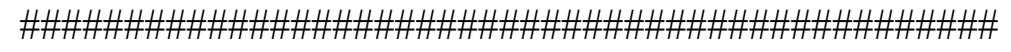

$\begin{array}{lc}\text { \&BMHFTD } & \\ \text { ATOMS } & \mathrm{Na} \mathrm{Cl} \\ \text { A } & 4.4430000000000000 \mathrm{E}+01 \\ \text { B } & 3.0000000000000000 \mathrm{E}+00 \\ \text { C } & 2.9710000000000000 \mathrm{E}-01 \\ \text { D } & 3.7850000000000000 \mathrm{E}-01 \\ \text { BD } & 3.0000000000000000 \mathrm{E}+00 \\ \text { RCUT } & 1.0000000000000000 \mathrm{E}+01 \\ \text { \&END BMHFTD } & \end{array}$

\section{\&END NONBONDED}

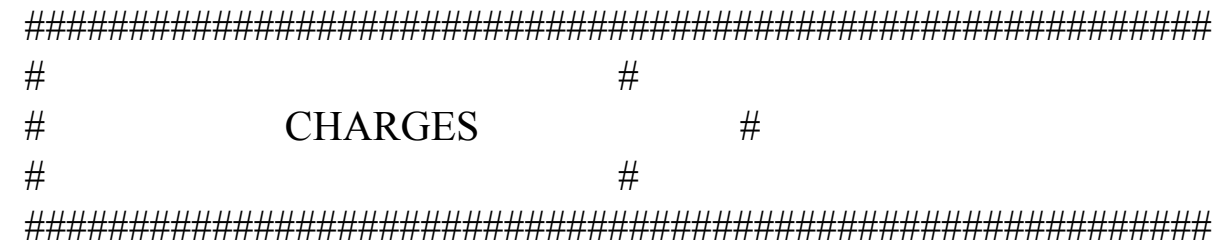

\section{\&CHARGE}

ATOM Na

CHARGE $\quad+1.0000$

\&END CHARGE

\&CHARGE

ATOM

CHARGE

$\mathrm{Cl}$

\&END CHARGE

$-1.0000$

\#

\&CHARGE

atom

Al

CHARGE

\&END CHARGE

$\&$ CHARGE

ATOM

CHARGE

$\mathrm{MgA}$

$+2.0000$

\&END CHARGE

$\&$ CHARGE atom

$\mathrm{Si}$ 


$\begin{array}{lcc}\text { CHARGE } & & +4.0000 \\ \text { \&END CHARGE } & & \\ \text { \&CHARGE } & & \\ \text { atom } & \mathrm{O} & -2.0000 \\ \text { CHARGE } & & \\ \text { \&END CHARGE } & & \\ \text { \&CHARGE } & \mathrm{OH} \\ \text { atom } & -1.1017 \\ \text { CHARGE } & \\ \text { \&END CHARGE } & \\ \text { \&CHARGE } & \\ \text { atom } & +0.1017 \\ \text { CHARGE } & \\ \text { \&END CHARGE } & \\ \text { \&CHARGE } & \\ \text { ATOM } & \mathrm{OW} \\ \text { CHARGE } & +0.0000 \\ \text { \&END CHARGE } & \\ \text { \&CHARGE } & \\ \text { ATOM } & \mathrm{HW} \\ \text { CHARGE } & +0.5190 \\ \text { \&END CHARGE } & \\ \text { \&CHARGE } & \\ \text { ATOM } & \mathrm{MW} \\ \text { CHARGE } & -1.0380 \\ \text { \&END CHARGE } & \end{array}$

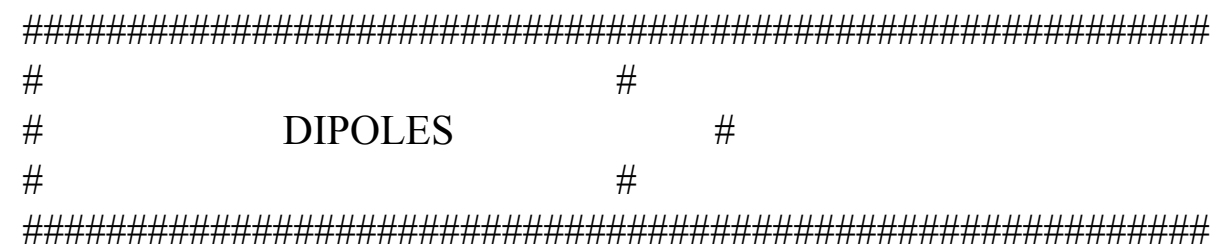

\&DIPOLE

$\begin{array}{ll}\text { ATOM } & \text { MW } \\ \text { APOL } & 1.4440000000000002 E+00\end{array}$

\&DAMPING

ORDER 4

TYPE Tang-Toennies

ATOM $\mathrm{Na}$

BIJ $\quad 1.5617000000000001 \mathrm{E}+00$

CIJ 6.8389999999999995E-01

\&END DAMPING

\&DAMPING

ORDER

TYPE

4

ATOM

Tang-Toennies

BIJ

$\mathrm{MgA}$

CIJ

$3.9964789111 \mathrm{E}+00$

\&END DAMPING

\&DAMPING

ORDER 


\begin{tabular}{|c|c|}
\hline TYPE & Tang-Toennies \\
\hline ATOM & $\mathrm{Al}$ \\
\hline BIJ & $3.3361958663 \mathrm{E}+00$ \\
\hline CIJ & $1.4999595717 \mathrm{E}+00$ \\
\hline \multicolumn{2}{|c|}{ \&END DAMPING } \\
\hline \multicolumn{2}{|c|}{ \&DAMPING } \\
\hline ORDER & 4 \\
\hline TYPE & Tang-Toennies \\
\hline ATOM & $\mathrm{Si}$ \\
\hline BIJ & $5.0005669339 \mathrm{E}+00$ \\
\hline CIJ & $4.9955556347 \mathrm{E}+00$ \\
\hline \multicolumn{2}{|c|}{ \&END DAMPING } \\
\hline \multicolumn{2}{|c|}{ \&DAMPING } \\
\hline ORDER & 4 \\
\hline TYPE & Tang-Toennies \\
\hline ATOM & $\mathrm{O}$ \\
\hline BIJ & $5.0199966566 \mathrm{E}+00$ \\
\hline CIJ & $4.9994813561 \mathrm{E}+00$ \\
\hline \multicolumn{2}{|c|}{ \&END DAMPING } \\
\hline \multicolumn{2}{|c|}{ \&DAMPING } \\
\hline ORDER & 4 \\
\hline TYPE & Tang-Toennies \\
\hline ATOM & $\mathrm{OH}$ \\
\hline BIJ & $5.0400021733 \mathrm{E}+00$ \\
\hline CIJ & $2.8582632299 \mathrm{E}+00$ \\
\hline \multicolumn{2}{|c|}{ \&END DAMPING } \\
\hline \multicolumn{2}{|c|}{ \&DAMPING } \\
\hline ORDER & 4 \\
\hline TYPE & Tang-Toennies \\
\hline ATOM & $\mathrm{H}$ \\
\hline BIJ & $1.5989661001 \mathrm{E}+00$ \\
\hline CIJ & $7.3792458212 \mathrm{E}-01$ \\
\hline \multicolumn{2}{|c|}{ \&END DAMPING } \\
\hline \multicolumn{2}{|c|}{ \&END DIPOLE } \\
\hline \multicolumn{2}{|l|}{$\&$ DIPOLE } \\
\hline ATOM & $\mathrm{Na}$ \\
\hline APOL & $0.18000000000000000 \mathrm{E}+00$ \\
\hline \multicolumn{2}{|c|}{ \&DAMPING } \\
\hline ORDER & 4 \\
\hline TYPE & Tang-Toennies \\
\hline ATOM & $\mathrm{Cl}$ \\
\hline BIJ & $5.0100031037 \mathrm{E}+00$ \\
\hline CIJ & $4.9999760983 \mathrm{E}+00$ \\
\hline \multicolumn{2}{|c|}{ \&END DAMPING } \\
\hline \multicolumn{2}{|c|}{ \&DAMPING } \\
\hline ORDER & 4 \\
\hline TYPE & Tang-Toennies \\
\hline ATOM & $\mathrm{Al}$ \\
\hline BIJ & $3.7020804884 \mathrm{E}+00$ \\
\hline CIJ & $3.3441736451 \mathrm{E}+00$ \\
\hline
\end{tabular}




\section{\&DAMPING}

ORDER

TYPE

ATOM

BIJ

CIJ

\&END DAMPING

\&DAMPING

ORDER

TYPE

ATOM

BIJ

CIJ
4

Tang-Toennies

$\mathrm{Si}$

$3.6630744028 \mathrm{E}+00$

$3.7737726208 \mathrm{E}+00$

\section{\&END DAMPING}

\&DAMPING

ORDER

TYPE

ATOM

4

Tang-Toennies

$\mathrm{MgA}$

$2.1495309938 \mathrm{E}+00$

$1.2791303578 \mathrm{E}-02$

BIJ

4

Tang-Toennies

$\mathrm{O}$

CIJ

$3.6478668866 \mathrm{E}+00$

$1.7620443832 \mathrm{E}+00$

\&END DAMPING

\&DAMPING

$\begin{array}{lc}\text { ORDER } & 4 \\ \text { TYPE } & \text { Tang-Toennies } \\ \text { ATOM } & \text { OH } \\ \text { BIJ } & 3.0092144512 \mathrm{E}+00 \\ \text { CIJ } & 4.8703709043 \mathrm{E}+00 \\ \text { \&END DAMPING } \\ \text { \&DAMPING } & \\ \text { ORDER } & 4 \\ \text { TYPE } & \text { Tang-Toennies } \\ \text { ATOM } & \mathrm{H} \\ \text { BIJ } & 2.7164978400 \mathrm{E}+00 \\ \text { CIJ } & 3.9161133919 \mathrm{E}+00\end{array}$

\&END DAMPING

\&DAMPING

ORDER

TYPE

4

ATOM

Tang-Toennies

HW

BIJ

$1.5988515783 \mathrm{E}+00$

CIJ

6.0039904460E-02

\&END DAMPING

\&DAMPING

ORDER

TYPE

4

ATOM

Tang-Toennies

MW

BIJ

$5.0100031037 \mathrm{E}+00$

CIJ

$4.9999760983 \mathrm{E}+00$

\&END DAMPING

$\&$ END DIPOLE

\&DIPOLE 


\begin{tabular}{|c|c|}
\hline ATOM & $\mathrm{Cl}$ \\
\hline APOL & $3.5000000000 \mathrm{E}+00$ \\
\hline \multicolumn{2}{|c|}{ \&DAMPING } \\
\hline ORDER & 4 \\
\hline TYPE & Tang-Toennies \\
\hline ATOM & $\mathrm{Na}$ \\
\hline BIJ & $2.7750000000 \mathrm{E}+00$ \\
\hline CIJ & $2.0400000000 \mathrm{E}+00$ \\
\hline \multicolumn{2}{|c|}{ \&END DAMPING } \\
\hline \multicolumn{2}{|c|}{ \&DAMPING } \\
\hline ORDER & 4 \\
\hline TYPE & Tang-Toennies \\
\hline ATOM & $\mathrm{Al}$ \\
\hline BIJ & $2.8860000000 \mathrm{E}+00$ \\
\hline CIJ & $2.1130000000 \mathrm{E}+00$ \\
\hline \multicolumn{2}{|c|}{ \&END DAMPING } \\
\hline \multicolumn{2}{|c|}{ \&DAMPING } \\
\hline ORDER & 4 \\
\hline TYPE & Tang-Toennies \\
\hline ATOM & $\mathrm{Si}$ \\
\hline BIJ & $2.8860000000 \mathrm{E}+00$ \\
\hline CIJ & $2.1130000000 \mathrm{E}+00$ \\
\hline \multicolumn{2}{|c|}{ \&END DAMPING } \\
\hline \multicolumn{2}{|c|}{ \&DAMPING } \\
\hline ORDER & 4 \\
\hline TYPE & Tang-Toennies \\
\hline ATOM & $\mathrm{MgA}$ \\
\hline BIJ & $2.8860000000 \mathrm{E}+00$ \\
\hline CIJ & $2.1130000000 \mathrm{E}+00$ \\
\hline \multicolumn{2}{|c|}{ \&END DAMPING } \\
\hline \multicolumn{2}{|c|}{ \&DAMPING } \\
\hline ORDER & 4 \\
\hline TYPE & Tang-Toennies \\
\hline ATOM & $\mathrm{O}$ \\
\hline BIJ & $2.4440000000 \mathrm{E}+00$ \\
\hline CIJ & $-1.9010000000 \mathrm{E}+00$ \\
\hline \multicolumn{2}{|c|}{ \&END DAMPING } \\
\hline \multicolumn{2}{|c|}{ \&DAMPING } \\
\hline ORDER & 4 \\
\hline TYPE & Tang-Toennies \\
\hline ATOM & $\mathrm{OH}$ \\
\hline BIJ & $2.4440000000 \mathrm{E}+00$ \\
\hline CIJ & $-1.9010000000 \mathrm{E}+00$ \\
\hline \multicolumn{2}{|c|}{ \&END DAMPING } \\
\hline \multicolumn{2}{|c|}{ \&DAMPING } \\
\hline ORDER & 4 \\
\hline TYPE & Tang-Toennies \\
\hline ATOM & $\mathrm{H}$ \\
\hline BIJ & $4.7940000000 \mathrm{E}+00$ \\
\hline CIJ & $1.0930000000 \mathrm{E}+00$ \\
\hline \multicolumn{2}{|c|}{ \&END DAMPING } \\
\hline \&DAMPI & \\
\hline
\end{tabular}




$\begin{array}{lc}\text { ORDER } & 4 \\ \text { TYPE } & \text { Tang-Toennies } \\ \text { ATOM } & \text { MW } \\ \text { BIJ } & 2.4440000000 \mathrm{E}+00 \\ \text { CIJ } & -1.9010000000 \mathrm{E}+00 \\ \text { \&END DAMPING } \\ \text { \&DAMPING } & \\ \text { ORDER } & 4 \\ \text { TYPE } & \text { Tang-Toennies } \\ \text { ATOM } & \text { HW } \\ \text { BIJ } & 4.7940000000 \mathrm{E}+00 \\ \text { CIJ } & 1.0930000000 \mathrm{E}+00\end{array}$

\&END DAMPING

\&END DIPOLE

\&DIPOLE

$\begin{array}{lc}\text { ATOM } & \text { O } \\ \text { APOL } & 9.0678561600 \mathrm{E}-01 \\ \text { \&DAMPING } & \\ \text { ORDER } & 4 \\ \text { TYPE } & \text { Tang-Toennies } \\ \text { ATOM } & \text { Na } \\ \text { BIJ } & 4.3304348490 \mathrm{E}+00 \\ \text { CIJ } & 2.1329271941 \mathrm{E}+00 \\ \text { \&END DAMPING } \\ \text { \&DAMPING } & \\ \text { ORDER } & 4 \\ \text { TYPE } & \text { Tang-Toennies } \\ \text { ATOM } & \text { Al } \\ \text { BIJ } & 4.7547803622 \mathrm{E}+00 \\ \text { CIJ } & 4.7328102147 \mathrm{E}+00\end{array}$

\&END DAMPING

\&DAMPING

$\begin{array}{lc}\text { ORDER } & 4 \\ \text { TYPE } & \text { Tang-Toennies } \\ \text { ATOM } & \text { MgA } \\ \text { BIJ } & 4.4714171244 \mathrm{E}+00 \\ \text { CIJ } & 4.9960034166 \mathrm{E}+00 \\ \text { \&END DAMPING } \\ \text { \&DAMPING } & \\ \text { ORDER } & 4 \\ \text { TYPE } & \text { Tang-Toennies } \\ \text { ATOM } & \text { Si } \\ \text { BIJ } & 5.0573402695 \mathrm{E}+00 \\ \text { CIJ } & 3.1653367044 \mathrm{E}+00 \\ \text { \&END DAMPING } & \\ \text { \&DAMPING } & \\ \text { ORDER } & 4 \\ \text { TYPE } & \text { Tang-Toennies } \\ \text { ATOM } & \text { O } \\ \text { BIJ } & 5.3846566789 \mathrm{E}+00 \\ \text { CIJ } & 4.9997463406 \mathrm{E}+00\end{array}$




$\begin{array}{lc}\text { \&END DAMPING } & \\ \text { \&DAMPING } & \\ \text { ORDER } & 4 \\ \text { TYPE } & \text { Tang-Toennies } \\ \text { ATOM } & \text { H } \\ \text { BIJ } & 2.0020662418 \mathrm{E}+00 \\ \text { CIJ } & 1.8606770135 \mathrm{E}+00 \\ \text { \&END DAMPING } & \\ \text { \&DAMPING } & \\ \text { ORDER } & 4 \\ \text { TYPE } & \text { Tang-Toennies } \\ \text { ATOM } & \text { HW } \\ \text { BIJ } & 1.5007242676 \mathrm{E}+00 \\ \text { CIJ } & 7.0000355520 \mathrm{E}-02 \\ \text { \&END DAMPING } & \\ \text { \&DAMPING } & \\ \text { ORDER } & 4 \\ \text { TYPE } & \text { Tang-Toennies } \\ \text { ATOM } & \text { MW } \\ \text { BIJ } & 5.1561158104 \mathrm{E}+00 \\ \text { CIJ } & 1.8434131234 \mathrm{E}+00\end{array}$

\&END DAMPING

\&END DIPOLE

\begin{tabular}{|c|c|}
\hline \multicolumn{2}{|c|}{ \&DIPOLE } \\
\hline ATOM & $\mathrm{OH}$ \\
\hline APOL & $2.3869000000 \mathrm{E}+00$ \\
\hline \multicolumn{2}{|c|}{ \&DAMPING } \\
\hline ORDER & 4 \\
\hline TYPE & Tang-Toennies \\
\hline ATOM & $\mathrm{Na}$ \\
\hline BIJ & $5.6778270635 \mathrm{E}+00$ \\
\hline CIJ & $3.4683380384 \mathrm{E}+00$ \\
\hline \multicolumn{2}{|c|}{ \&END DAMPING } \\
\hline \multicolumn{2}{|c|}{ \&DAMPING } \\
\hline ORDER & 4 \\
\hline TYPE & Tang-Toennies \\
\hline ATOM & $\mathrm{Al}$ \\
\hline BIJ & $3.3669896020 \mathrm{E}+00$ \\
\hline CIJ & $1.6228765714 \mathrm{E}+00$ \\
\hline \multicolumn{2}{|c|}{ \&END DAMPING } \\
\hline \multicolumn{2}{|c|}{ \&DAMPING } \\
\hline ORDER & 4 \\
\hline TYPE & Tang-Toennies \\
\hline ATOM & $\mathrm{MgA}$ \\
\hline BIJ & $3.0622874458 \mathrm{E}+00$ \\
\hline CIJ & $1.4544602067 \mathrm{E}+00$ \\
\hline \multicolumn{2}{|c|}{ \&END DAMPING } \\
\hline \multicolumn{2}{|c|}{ \&DAMPING } \\
\hline ORDER & 4 \\
\hline TYPE & Tang-Toennies \\
\hline ATOM & $\mathrm{Si}$ \\
\hline
\end{tabular}




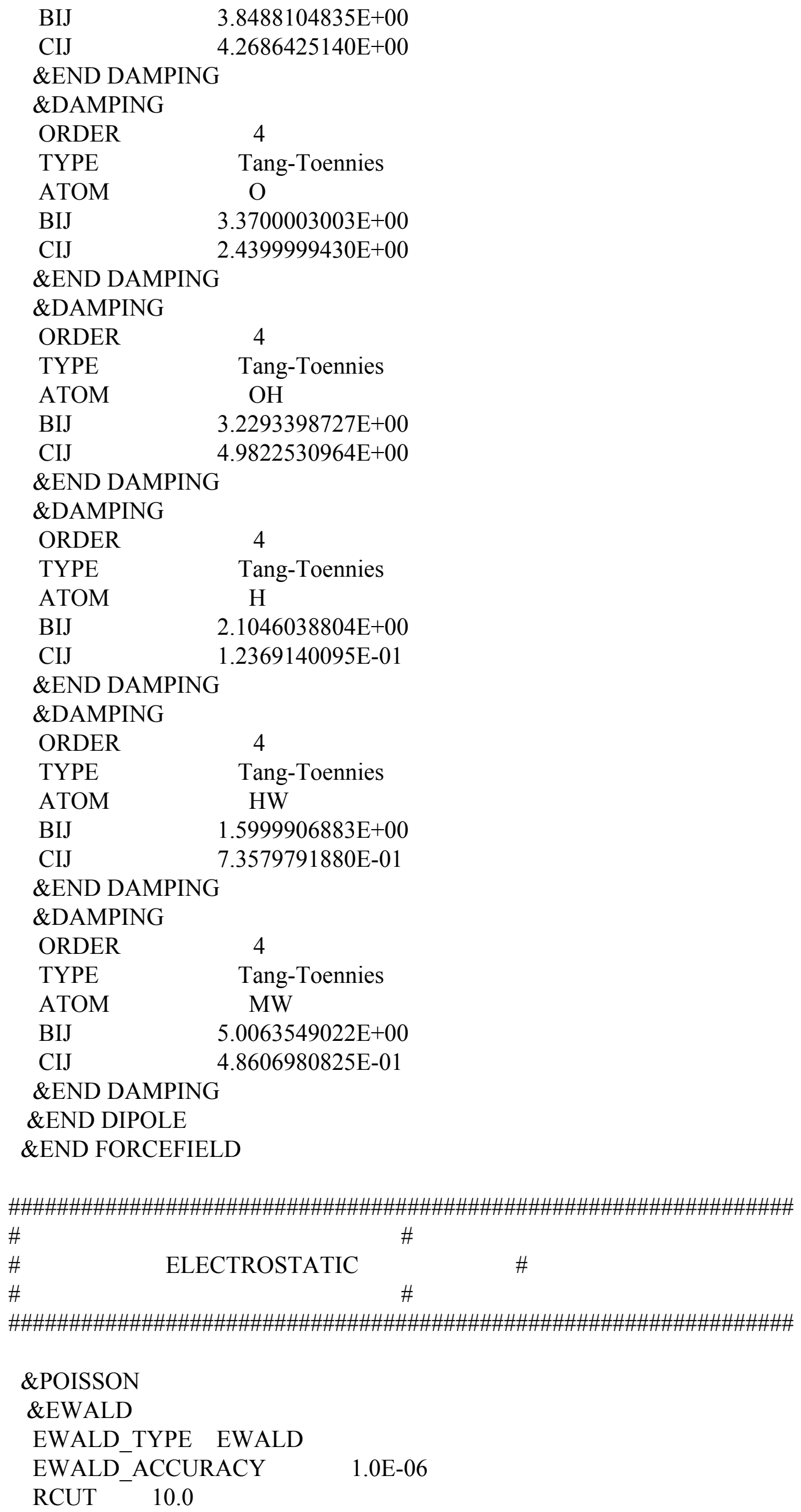




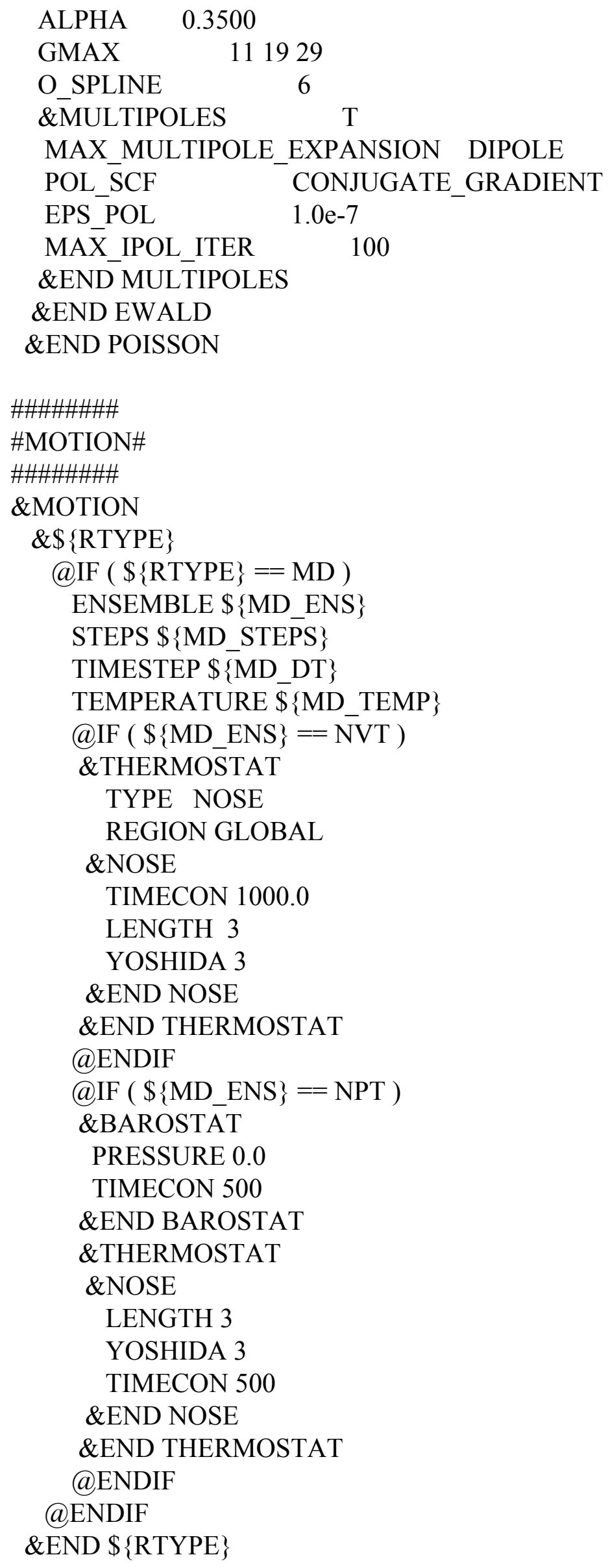




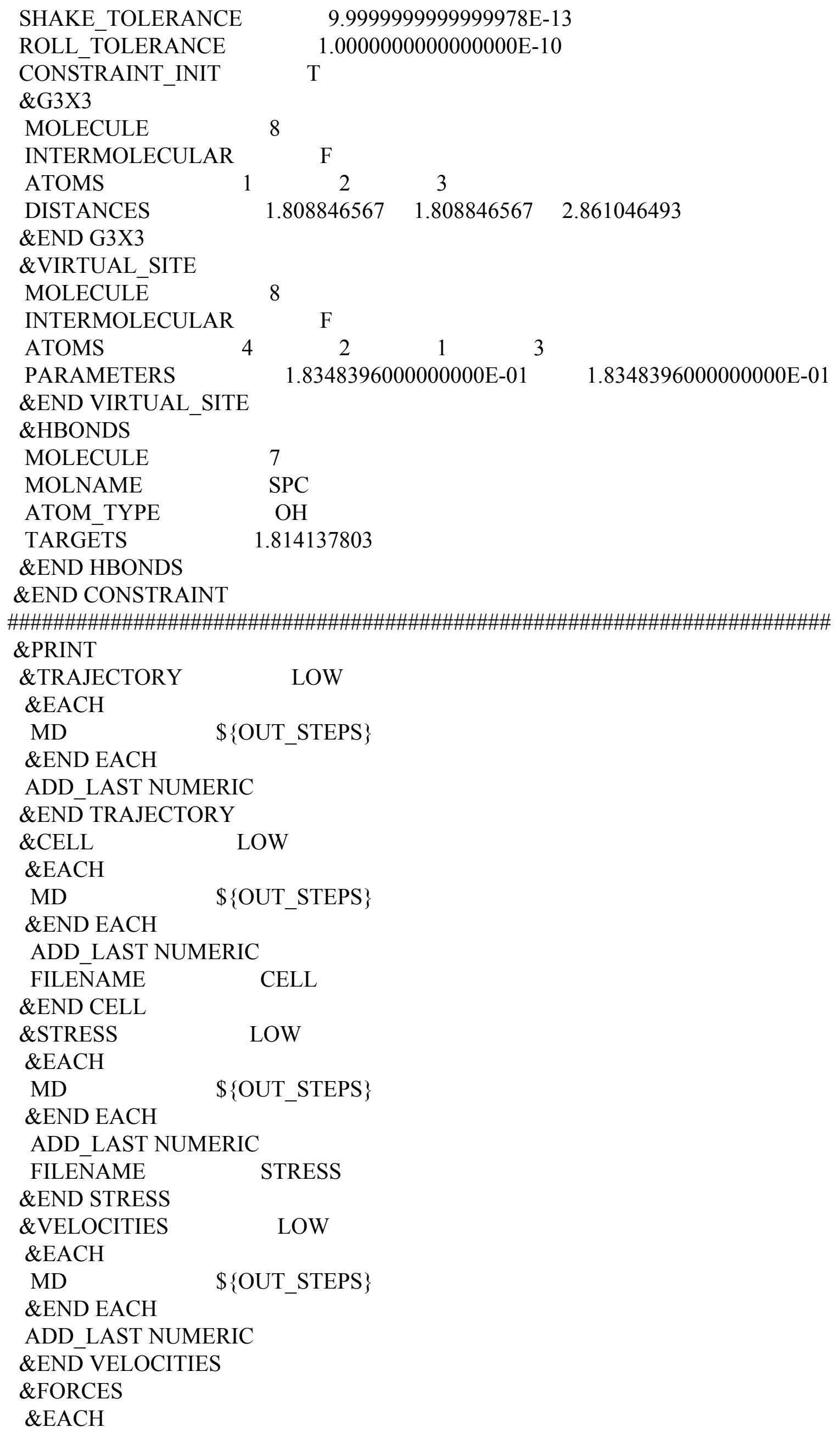


\&END EACH

ADD_LAST NUMERIC

\&END FORCES

\&END PRINT

$\&$ END MOTION

\section{\#\#\#\#\#\#\#}

\#SUBSYS\#

\#\#\#\#\#\#\#

\section{\&SUBSYS}

$\&$ CELL

$\mathrm{ABC}$

$20.72 \quad 35.88 \quad 54.7084$

ALPHA_BETA_GAMMA $\quad 90.00 \quad 90.00 \quad 90.00$

PERIODIC XȲZ

MULTIPLE_UNIT_CELL_ 111

\&END CELL

\begin{tabular}{|c|c|}
\hline$\&$ KIND & $\mathrm{Na}$ \\
\hline ELEMENT & $\mathrm{Na}$ \\
\hline MASS & 22.9898 \\
\hline \multicolumn{2}{|l|}{ \&END KIND } \\
\hline$\&$ KIND & $\mathrm{Cl}$ \\
\hline ELEMENT & $\mathrm{Cl}$ \\
\hline MASS & 35.453 \\
\hline \multicolumn{2}{|l|}{ \&END KIND } \\
\hline \&KIND & $\mathrm{Al}$ \\
\hline ELEMENT & $\mathrm{Al}$ \\
\hline MASS & 26.9820 \\
\hline \multicolumn{2}{|l|}{ \&END KIND } \\
\hline \&KIND & $\mathrm{MgA}$ \\
\hline ELEMENT & $\mathrm{Mg}$ \\
\hline MASS & $24.3050 \mathrm{ZZ}$ \\
\hline \multicolumn{2}{|l|}{ \&END KIND } \\
\hline$\&$ KIND & $\mathrm{Si}$ \\
\hline ELEMENT & $\mathrm{Si}$ \\
\hline MASS & 28.0855 \\
\hline \multicolumn{2}{|l|}{ \&END KIND } \\
\hline$\&$ KIND & $\mathrm{O}$ \\
\hline ELEMENT & $\mathrm{O}$ \\
\hline MASS & 15.9994 \\
\hline \multicolumn{2}{|l|}{ \&END KIND } \\
\hline$\&$ KIND & $\mathrm{OH}$ \\
\hline ELEMENT & $\mathrm{O}$ \\
\hline MASS & 15.9994 \\
\hline \multicolumn{2}{|l|}{ \&END KIND } \\
\hline \&KIND & $\mathrm{H}$ \\
\hline ELEMENT & $\mathrm{H}$ \\
\hline MASS & 1.0080 \\
\hline
\end{tabular}




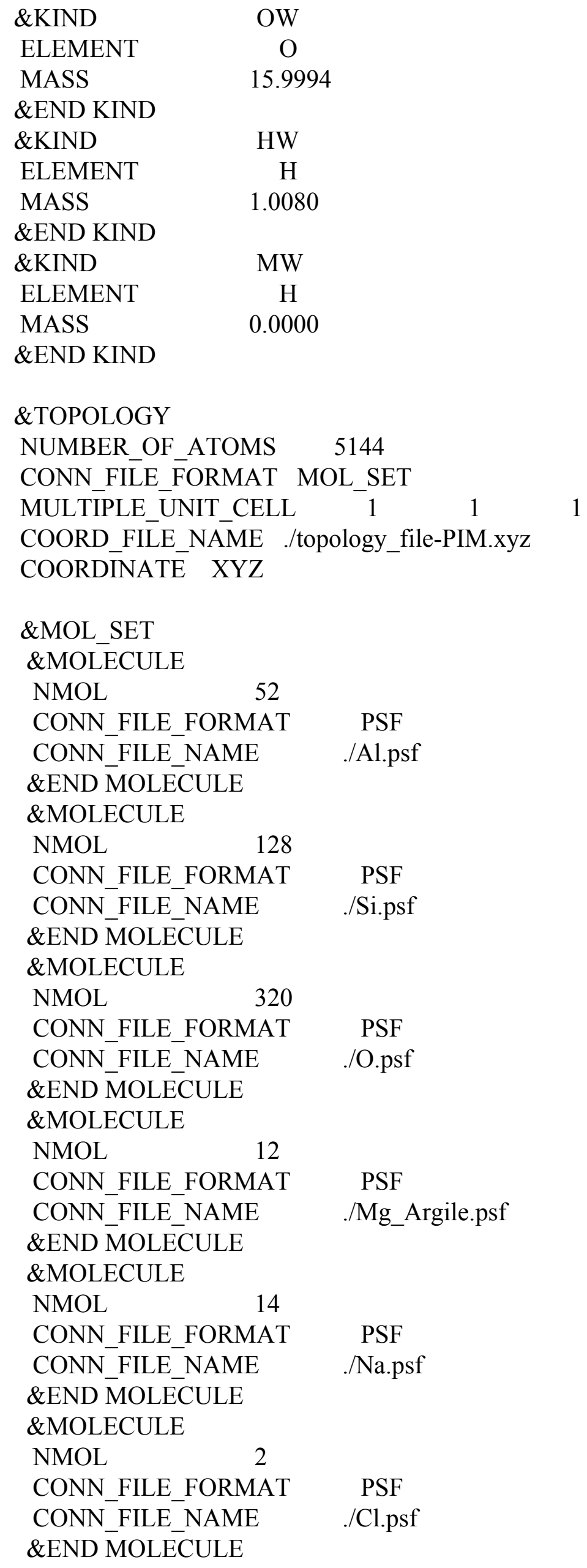


\&MOLECULE

NMOL

64

CONN_FILE_FORMAT

PSF

CONN FILE NAME

./OH.psf

\&END MOLECULE

\&MOLECULE

NMOL

CONN_FILE_FORMAT

PSF

CONN_FILE_NAME

./Eau_DC.psf

\&END MOLECULE

\&END MOL SET

\&END TOPOLOGY

\&END SUBSYS

\&END FORCE_EVAL 
\#Topology file

4022

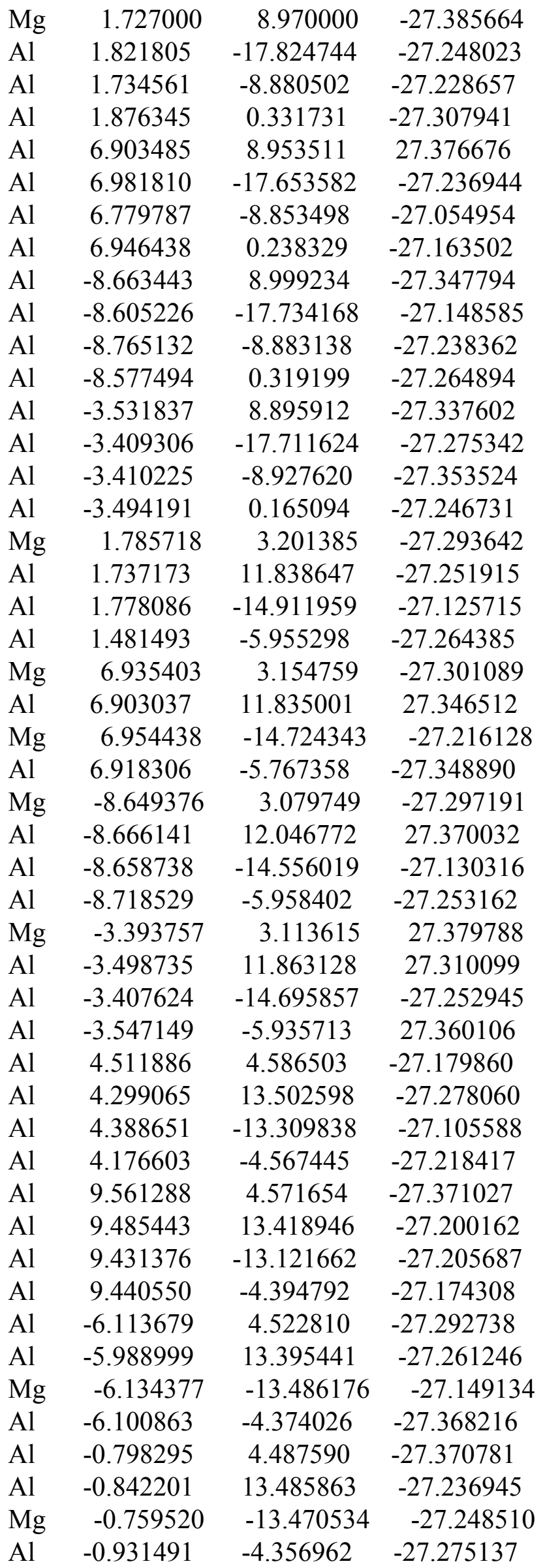




\begin{tabular}{|c|c|c|c|}
\hline $\mathrm{Al}$ & 4.270581 & 7.535046 & -27.324606 \\
\hline $\mathrm{Al}$ & 4.454378 & 16.452853 & -27.335641 \\
\hline $\mathrm{Mg}$ & 4.285482 & -10.377375 & -27.166359 \\
\hline $\mathrm{Al}$ & 4.407095 & -1.576443 & -27.311952 \\
\hline $\mathrm{Mg}$ & 9.477853 & 7.440499 & -27.314405 \\
\hline $\mathrm{Al}$ & 9.469713 & 16.455248 & -27.303186 \\
\hline $\mathrm{Mg}$ & 9.491126 & -10.283448 & -27.297412 \\
\hline $\mathrm{Al}$ & 9.495623 & -1.460178 & -27.329773 \\
\hline $\mathrm{Mg}$ & -6.136255 & 7.539339 & -27.338854 \\
\hline $\mathrm{Al}$ & -6.045155 & 16.638848 & -27.277659 \\
\hline $\mathrm{Al}$ & -6.064166 & -10.522156 & -27.364682 \\
\hline $\mathrm{Al}$ & -6.014344 & -1.415467 & -27.280001 \\
\hline $\mathrm{Al}$ & -0.895635 & 7.569196 & -27.248619 \\
\hline $\mathrm{Al}$ & -0.853595 & 16.615874 & -27.288403 \\
\hline $\mathrm{Al}$ & -0.912769 & -10.423696 & -27.329463 \\
\hline $\mathrm{Al}$ & -0.899108 & -1.236982 & -27.194405 \\
\hline $\mathrm{Si}$ & -0.066631 & 3.120980 & -24.621231 \\
\hline $\mathrm{Si}$ & -0.037033 & 12.031987 & -24.622847 \\
\hline $\mathrm{Si}$ & -0.009343 & -14.879481 & -24.516534 \\
\hline $\mathrm{Si}$ & -0.102494 & -5.735248 & -24.543440 \\
\hline $\mathrm{Si}$ & 5.192543 & 3.179271 & -24.551767 \\
\hline $\mathrm{Si}$ & 5.125104 & 12.209333 & -24.507644 \\
\hline $\mathrm{Si}$ & 5.102783 & -14.695918 & -24.429117 \\
\hline $\mathrm{Si}$ & 5.127413 & -5.856535 & -24.544243 \\
\hline $\mathrm{Si}$ & 10.346405 & 3.127110 & -24.663644 \\
\hline $\mathrm{Si}$ & 10.307448 & 12.197291 & -24.374881 \\
\hline $\mathrm{Si}$ & -10.352643 & -14.726793 & -24.552792 \\
\hline $\mathrm{Si}$ & 10.167875 & -5.744359 & -24.514311 \\
\hline $\mathrm{Si}$ & -5.209391 & 3.232875 & -24.546530 \\
\hline $\mathrm{Si}$ & -5.213723 & 12.013468 & -24.598964 \\
\hline $\mathrm{Si}$ & -5.165216 & -14.736237 & -24.561321 \\
\hline $\mathrm{Si}$ & -5.307624 & -5.790408 & -24.560733 \\
\hline $\mathrm{Si}$ & 0.042479 & 6.064676 & -24.667775 \\
\hline $\mathrm{Si}$ & -0.123942 & 15.006807 & -24.404745 \\
\hline $\mathrm{Si}$ & -0.082794 & -11.780048 & -24.598525 \\
\hline $\mathrm{Si}$ & -0.121516 & -2.859410 & -24.548666 \\
\hline $\mathrm{Si}$ & 5.183277 & 6.124677 & -24.471836 \\
\hline $\mathrm{Si}$ & 5.185441 & 15.179465 & -24.620144 \\
\hline $\mathrm{Si}$ & 5.023562 & -11.823024 & -24.336360 \\
\hline $\mathrm{Si}$ & 5.039035 & -2.885179 & -24.572839 \\
\hline $\mathrm{Si}$ & 10.274386 & 6.020223 & -24.679127 \\
\hline $\mathrm{Si}$ & 10.234930 & 15.179295 & -24.527577 \\
\hline $\mathrm{Si}$ & 10.259150 & -11.791599 & -24.560758 \\
\hline $\mathrm{Si}$ & 10.199026 & -2.793284 & -24.513528 \\
\hline $\mathrm{Si}$ & -5.279233 & 6.118107 & -24.613498 \\
\hline $\mathrm{Si}$ & -5.178140 & 15.009414 & -24.516617 \\
\hline $\mathrm{Si}$ & -5.347948 & -11.721872 & -24.533502 \\
\hline $\mathrm{Si}$ & -5.263662 & -2.872064 & -24.531826 \\
\hline $\mathrm{Si}$ & 2.565646 & 1.662735 & -24.557639 \\
\hline $\mathrm{Si}$ & 2.515798 & 10.588484 & -24.491481 \\
\hline $\mathrm{Si}$ & 2.565354 & -16.320330 & -24.411832 \\
\hline $\mathrm{Si}$ & 2.403041 & -7.364385 & -24.501809 \\
\hline
\end{tabular}




\begin{tabular}{|c|c|c|c|}
\hline $\mathrm{Si}$ & 7.662933 & 1.629760 & -24.415765 \\
\hline $\mathrm{Si}$ & 7.703764 & 10.605554 & -24.622879 \\
\hline $\mathrm{Si}$ & 7.688874 & -16.226848 & -24.470035 \\
\hline $\mathrm{Si}$ & 7.681271 & -7.339957 & -24.540532 \\
\hline $\mathrm{Si}$ & -7.844426 & 1.715425 & -24.538936 \\
\hline $\mathrm{Si}$ & -7.935939 & 10.585908 & -24.533785 \\
\hline $\mathrm{Si}$ & -7.686928 & -16.361477 & -24.471371 \\
\hline $\mathrm{Si}$ & -8.005732 & -7.314352 & -24.582171 \\
\hline $\mathrm{Si}$ & -2.659674 & 1.608570 & -24.525062 \\
\hline $\mathrm{Si}$ & -2.652451 & 10.530857 & -24.603116 \\
\hline $\mathrm{Si}$ & -2.661045 & -16.170709 & -24.501361 \\
\hline $\mathrm{Si}$ & -2.808913 & -7.346083 & -24.527355 \\
\hline $\mathrm{Si}$ & 2.616850 & 7.623570 & -24 \\
\hline $\mathrm{Si}$ & 2.505950 & 16.590163 & 9421 \\
\hline $\mathrm{Si}$ & 2.385622 & -10.254047 & -24.456422 \\
\hline $\mathrm{Si}$ & 2.553428 & -1.347228 & -24.521084 \\
\hline $\mathrm{Si}$ & 7.694813 & 7.673835 & -24.644374 \\
\hline $\mathrm{Si}$ & 7.702173 & 16.774742 & 1387 \\
\hline $\mathrm{Si}$ & 7.632225 & -10.237049 & 47822 \\
\hline $\mathrm{Si}$ & 7.639515 & -1.278252 & 3087 \\
\hline $\mathrm{Si}$ & -7.851002 & 7.623855 & -24 \\
\hline $\mathrm{Si}$ & -7.785060 & 16.596693 & -24.507200 \\
\hline $\mathrm{Si}$ & -7.864095 & -10.225666 & 3936 \\
\hline $\mathrm{Si}$ & -7.846411 & -1.290708 & -24 \\
\hline $\mathrm{Si}$ & -2.563488 & 7.502781 & -24.590911 \\
\hline $\mathrm{Si}$ & -2.552866 & 16.683308 & -24.411388 \\
\hline $\mathrm{Si}$ & -2.711962 & -10.348233 & -24.568164 \\
\hline $\mathrm{Si}$ & -2.640032 & -1.295311 & -24.502482 \\
\hline $\mathrm{Si}$ & 0.967371 & & \\
\hline $\mathrm{Si}$ & 0.964762 & 10.439612 & 5494 \\
\hline $\mathrm{Si}$ & 1.025807 & -16.480485 & 8655 \\
\hline $\mathrm{Si}$ & 0.859195 & -7.457402 & 1240 \\
\hline $\mathrm{Si}$ & 6.172957 & 1.532136 & 079 \\
\hline $\mathrm{Si}$ & 6.056885 & 10.394101 & 2487 \\
\hline $\mathrm{Si}$ & 6.107751 & -16.390885 & 9263 \\
\hline $\mathrm{Si}$ & 6.036523 & -7.366260 & 0858 \\
\hline $\mathrm{Si}$ & -9.411121 & 1.455703 & 3841 \\
\hline $\mathrm{Si}$ & -9.452167 & 10.539124 & 24.596368 \\
\hline $\mathrm{Si}$ & -9.513011 & -16.429684 & 24.813373 \\
\hline $\mathrm{Si}$ & -9.483727 & -7.419315 & 8727 \\
\hline $\mathrm{Si}$ & -4.214257 & 1.485815 & 24.763323 \\
\hline $\mathrm{Si}$ & -4.214751 & 10.392149 & 24.648225 \\
\hline $\mathrm{Si}$ & -4.186946 & -16.430350 & 24.781346 \\
\hline $\mathrm{Si}$ & -4.271216 & -7.425251 & 24.660755 \\
\hline $\mathrm{Si}$ & 0.822022 & 4.475980 & 24.791922 \\
\hline $\mathrm{Si}$ & 0.874375 & 13.433366 & 24.782568 \\
\hline $\mathrm{Si}$ & 0.941326 & -13.482368 & 24.938058 \\
\hline $\mathrm{Si}$ & 0.939712 & -4.575962 & 24.756349 \\
\hline $\mathrm{Si}$ & 5.969644 & 4.412226 & 24.764026 \\
\hline $\mathrm{Si}$ & 6.091818 & 13.392500 & 24.660315 \\
\hline $\mathrm{Si}$ & 6.041857 & -13.324200 & 24.947764 \\
\hline $\mathrm{Si}$ & 5.988865 & -4.474104 & 24.653564 \\
\hline
\end{tabular}




\begin{tabular}{|c|c|c|c|}
\hline $\mathrm{Si}$ & -9.555859 & 4.404123 & 9788 \\
\hline C: & -9.490275 & 13.474794 & 24.680700 \\
\hline & -9.497117 & -13.374247 & 24.879590 \\
\hline & -9.447871 & -4.475199 & 24.760776 \\
\hline & -4.278585 & 4.526725 & 24.715821 \\
\hline & -4.333410 & 13.411114 & 24.746585 \\
\hline & -4.287229 & -13.517271 & 24.852516 \\
\hline & -4.267290 & -4.475875 & 24.718872 \\
\hline & 3.444882 & 0.032265 & 24.751084 \\
\hline & 3.506777 & 8.810306 & 24.708098 \\
\hline & 3.534772 & 17.853545 & 24.682970 \\
\hline & 3.411420 & -8.960123 & 24.857239 \\
\hline & 8.71 & -0.00 & 24. \\
\hline i & 8.600 & 8.98 & 24.6 \\
\hline & 8.58 & -17.9 & 215 \\
\hline & 8.63 & -8.96 & 24.8 \\
\hline & -6.83 & 0.0 & 24. \\
\hline & -6.8 & 8.9 & 24. \\
\hline & -6.90 & -17.8 & 994 \\
\hline S & -6.9 & -8.9 & 17 \\
\hline & -1.7 & & \\
\hline $\mathrm{Si}$ & -1.68 & 8.97 & 24. \\
\hline & -1.6 & 17.8 & 510 \\
\hline & -1.79 & -8.9 & 677 \\
\hline $\mathrm{Si}$ & 3.5 & 5.9 & 24. \\
\hline & & & \\
\hline $\mathrm{Si}$ & 3.43 & -11.9 & 780 \\
\hline $\mathrm{Si}$ & 3.46 & -2.96 & 066 \\
\hline & 8.68 & 5.9 & 24.5 \\
\hline S1 & 8.6 & 14. & 375 \\
\hline $\mathrm{Si}$ & & -11.9 & \\
\hline $\mathrm{Si}$ & 8.67 & -3.0 & 24. \\
\hline $\mathrm{Si}$ & -6.8 & 5.9 & 24. \\
\hline & -6.8 & 75 & 422 \\
\hline S1 & -6.8 & -11.5 & 625 \\
\hline $\mathrm{Si}$ & -6.8 & & \\
\hline $\mathrm{Si}$ & -1.6 & 6.00 & 24.7 \\
\hline $\mathrm{Si}$ & -1.643446 & 15.0 & 3688 \\
\hline & -1.695828 & -11.903934 & 24.864682 \\
\hline 1 & -1.66 & -2.93 & $24 . ?$ \\
\hline 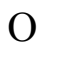 & 1.29 & 2.38 & -24.1 \\
\hline $\mathrm{O}$ & 1.210495 & 119 & -23.987883 \\
\hline $\mathrm{O}$ & 1.313203 & -15.546412 & -23.945675 \\
\hline $\mathrm{O}$ & 1.182130 & -6.539694 & -23.927734 \\
\hline $\mathrm{O}$ & 6.440175 & 2.472842 & -23.944138 \\
\hline $\mathrm{O}$ & 6.369640 & 11.345992 & -24.114164 \\
\hline $\mathrm{O}$ & 6.492184 & -15.334739 & -23.971944 \\
\hline $\mathrm{O}$ & 6.422717 & -6.504090 & -23.995246 \\
\hline 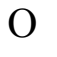 & -9.103191 & 2.444485 & -24.105817 \\
\hline $\mathrm{O}$ & -9.139522 & 11.447408 & -23.969202 \\
\hline & -9.021763 & -15.633370 & -23.911345 \\
\hline & -9.255202 & -6.467969 & -24.024328 \\
\hline
\end{tabular}




\begin{tabular}{|c|c|c|c|}
\hline $\mathrm{O}$ & -3.846809 & 2.495125 & -24.039150 \\
\hline $\mathrm{O}$ & -3.865373 & 11.331207 & -24.133948 \\
\hline $\mathrm{O}$ & -3.834112 & -15.376174 & -23.859066 \\
\hline $\mathrm{O}$ & -4.049332 & -6.571822 & -24.037965 \\
\hline $\mathrm{O}$ & 3.889402 & 2.345618 & -24.069448 \\
\hline $\mathrm{O}$ & 3.754467 & 11.512329 & -24.067820 \\
\hline $\mathrm{O}$ & 3.939632 & -15.635986 & 9037 \\
\hline $\mathrm{O}$ & 3.759797 & -6.585746 & 7043 \\
\hline $\mathrm{O}$ & 9.084121 & 2.262808 & -24.157810 \\
\hline $\mathrm{O}$ & 9.008615 & 11.314320 & -24.101371 \\
\hline $\mathrm{O}$ & 9.090727 & -15.546806 & 5750 \\
\hline $\mathrm{O}$ & 8.960010 & -6.5 & -24 \\
\hline $\mathrm{O}$ & -6.489157 & 2.3 & -24 \\
\hline $\mathrm{O}$ & -6.537740 & 11.296387 & 9051 \\
\hline $\mathrm{O}$ & -6.387189 & -15.604131 & 7625 \\
\hline $\mathrm{O}$ & -6.621982 & -6.605946 & 1893 \\
\hline $\mathrm{O}$ & -1.302644 & 2.26 & -24 \\
\hline $\mathrm{O}$ & -1.324442 & 11.227986 & 3047 \\
\hline $\mathrm{O}$ & -1.273931 & -15.587465 & 8009 \\
\hline $\mathrm{O}$ & -1.421082 & -6.5 & 991 \\
\hline $\mathrm{O}$ & 3.856135 & 6.81 & -23 . \\
\hline $\mathrm{O}$ & 3.894687 & 15.8 & 588 \\
\hline $\mathrm{O}$ & 3.737639 & -10.9 & 889 \\
\hline $\mathrm{O}$ & 3.76 & -2.1 & -23 \\
\hline $\mathrm{O}$ & 9.019354 & & \\
\hline $\mathrm{O}$ & 9.034444 & 4669 & 735 \\
\hline $\mathrm{O}$ & 9.010373 & -10.9 & 063 \\
\hline $\mathrm{O}$ & 8.958942 & -2.0 & -23 \\
\hline $\mathrm{O}$ & -6.520 & 6.9 & -24 \\
\hline $\mathrm{O}$ & -6.459810 & 15.8 & 031 \\
\hline $\mathrm{O}$ & -6.586277 & 2847 & 4949 \\
\hline $\mathrm{O}$ & -6.551789 & -2.0 & -24 \\
\hline $\mathrm{O}$ & -1.274645 & 224 & -24 \\
\hline $\mathrm{O}$ & -1.313932 & 967 & 999 \\
\hline $\mathrm{O}$ & -1.425929 & -11.0 & 287 \\
\hline $\mathrm{O}$ & -1.388690 & -2.0 & -23 \\
\hline $\mathrm{O}$ & 1.341297 & 6.90 & -24.1 \\
\hline $\mathrm{O}$ & 1.310378 & 15.656979 & 2620 \\
\hline $\mathrm{O}$ & 1.142910 & -11.067863 & 1921 \\
\hline $\mathrm{O}$ & & -2.0 & 656 \\
\hline $\mathrm{O}$ & 6.437733 & 6.942621 & -24.075244 \\
\hline $\mathrm{O}$ & 6.499324 & 15.896123 & -24 \\
\hline $\mathrm{O}$ & 6.425230 & -11.100052 & 9967 \\
\hline $\mathrm{O}$ & 6.415519 & -2.205265 & -24.3 \\
\hline $\mathrm{O}$ & -9.097659 & 6.778272 & -24.267002 \\
\hline $\mathrm{O}$ & -9.087465 & 15.798991 & -24.274333 \\
\hline $\mathrm{O}$ & -9.151050 & -11.014672 & -24.163616 \\
\hline $\mathrm{O}$ & -9.100357 & -2.181344 & -24.202086 \\
\hline $\mathrm{O}$ & -3.906748 & 6.773943 & -24.160395 \\
\hline $\mathrm{O}$ & -3.840716 & 15.740776 & -24.155391 \\
\hline $\mathrm{O}$ & -4.039427 & -11.058905 & -24.114716 \\
\hline $\mathrm{O}$ & -3.949155 & -2.120924 & -24.252779 \\
\hline
\end{tabular}




\begin{tabular}{|c|c|c|c|}
\hline $\mathrm{O}$ & -0.027461 & 4.627795 & -24.020156 \\
\hline $\mathrm{C}$ & -0.130123 & 13.525972 & -24.018241 \\
\hline & -0.078698 & -13.316897 & -24.102081 \\
\hline & -0.060178 & -4.299086 & -23.973604 \\
\hline$C$ & 5.195166 & 4.660006 & -23.995555 \\
\hline & 5.210102 & 13.696062 & -23.980910 \\
\hline $\mathrm{C}$ & 4.901210 & -13.272968 & -23.861754 \\
\hline C & 5.043651 & -4.376735 & -23.929748 \\
\hline & 10.289229 & 4.584426 & -24.125341 \\
\hline C & 10.250548 & 13.653917 & 37671 \\
\hline $\mathrm{C}$ & 10.337756 & -13.263667 & 21408 \\
\hline $\mathrm{C}$ & 10.1 & -4.291010 & 3746 \\
\hline 0 & -5.366522 & 4.675260 & -23.910102 \\
\hline $\mathrm{C}$ & -5.325059 & 13.541492 & 56728 \\
\hline $\mathrm{C}$ & -5.335823 & -13.225414 & 62327 \\
\hline C & -5.443748 & -4.339933 & 2835 \\
\hline $\mathrm{C}$ & 2.68 & 9.0 & -24 \\
\hline 0 & 2.423237 & -17.858651 & 7804 \\
\hline $\mathrm{C}$ & 2.447037 & -8.782533 & -23 \\
\hline C & 2.582922 & 0.124345 & -24 \\
\hline C & 7.743660 & 9.113346 & -24 \\
\hline C & 7.63 & -17.706982 & -23 \\
\hline $\mathrm{C}$ & 7.642962 & -8.796250 & -23 \\
\hline $\mathrm{O}$ & 7.5 & 0.13 & -23 \\
\hline $\mathrm{C}$ & -7.952884 & & \\
\hline $\mathrm{C}$ & -7.718876 & -17.860091 & 0657 \\
\hline $\mathrm{O}$ & -7.944625 & -8.749364 & -24 \\
\hline $\mathrm{C}$ & -7.95 & 0.120376 & -23 \\
\hline $\mathrm{O}$ & -2.5 & 9.0 & -24 \\
\hline C & -2.698659 & -17.732694 & 673 \\
\hline $\mathrm{C}$ & -2.726525 & -8.816267 & -24 \\
\hline $\mathrm{O}$ & -2.810328 & 0.201511 & -23 . \\
\hline C & 2.171195 & 0.73 & 24. \\
\hline $\mathrm{O}$ & 2.23 & 9.6 & 24 \\
\hline $\mathrm{O}$ & 2.250359 & -17.245583 & 577 \\
\hline $\mathrm{C}$ & 2.143 & -8.201600 & 24. \\
\hline $\mathrm{O}$ & 7.378035 & 0.770043 & 24. \\
\hline C & 7.287850 & 9.690159 & 23.9 \\
\hline $\mathrm{O}$ & 7.395022 & -17.105013 & 24. \\
\hline $\mathrm{O}$ & 7.335339 & -8.190041 & \\
\hline C & -8.147932 & 0.727698 & 24.2 \\
\hline $\mathrm{O}$ & -8.164118 & 9.771230 & 24.2 \\
\hline $\mathrm{O}$ & -8.176875 & -17.083396 & 1615 \\
\hline $\mathrm{O}$ & -8.232111 & -8.193073 & 24.206084 \\
\hline $\mathrm{O}$ & -2.938913 & 0.787507 & 24.142491 \\
\hline 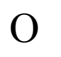 & -2.961442 & 9.653993 & 24.091472 \\
\hline $\mathrm{O}$ & -2.913644 & -17.222072 & 24.310967 \\
\hline 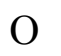 & -3.081049 & -8.285024 & 24.119539 \\
\hline $\mathrm{O}$ & 2.153353 & 5.229655 & 24.321601 \\
\hline 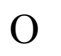 & 2.202586 & 14.226446 & 24.524668 \\
\hline 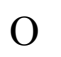 & 2.173208 & -12.675637 & 24.457813 \\
\hline $\mathrm{O}$ & 2.148156 & -3.687179 & 24.414831 \\
\hline
\end{tabular}




\begin{tabular}{|c|c|c|c|}
\hline$\Omega$ & 7.379323 & 5.176170 & 24.177600 \\
\hline $\mathrm{C}$ & 7.361754 & 14.151146 & 24.399446 \\
\hline $\mathrm{C}$ & 7.353196 & -12.643122 & 24.477405 \\
\hline & 7.344517 & -3.690620 & 24.358607 \\
\hline $\mathrm{C}$ & -8.185954 & 5.193638 & 24.375955 \\
\hline C & -8.129338 & 14.149539 & 24.499338 \\
\hline $\mathrm{C}$ & -8.158893 & -12.710049 & 24.315364 \\
\hline C & -8.107692 & -3.759644 & 24.406807 \\
\hline $\mathrm{C}$ & -3.005409 & 5.327683 & 24.259916 \\
\hline $\mathrm{O}$ & -2.908916 & 14.166063 & 24.498889 \\
\hline $\mathrm{C}$ & -3.025783 & -12.659421 & 24.459750 \\
\hline $\mathrm{O}$ & -3.016828 & -3.650534 & 24.486649 \\
\hline $\mathrm{O}$ & -0.390801 & 0.829108 & 0599 \\
\hline $\mathrm{C}$ & -0.414854 & 9.792033 & 8301 \\
\hline $\mathrm{C}$ & -0.330442 & -17.224953 & 24.449465 \\
\hline C & -0.492016 & -8.145912 & 24.356934 \\
\hline $\mathrm{O}$ & 4.8 & 0.7 & 5611 \\
\hline $\mathrm{O}$ & 4.769895 & & 80804 \\
\hline $\mathrm{O}$ & 4.865178 & -17.230402 & 24.438562 \\
\hline $\mathrm{C}$ & 4.722424 & -8.130263 & 24.507066 \\
\hline $\mathrm{O}$ & 10.001847 & 219 & 10516 \\
\hline $\mathrm{O}$ & 9.903851 & 9.8 & 9389 \\
\hline $\mathrm{O}$ & 9.962239 & -17.254843 & 15255 \\
\hline 0 & 9.979293 & -8.1 & 0883 \\
\hline $\mathrm{C}$ & -5.542974 & & 1858 \\
\hline $\mathrm{O}$ & -5.590348 & 9.64 & 24.319918 \\
\hline ) & -5.582842 & -17.147198 & 24.366503 \\
\hline $\mathrm{C}$ & -5.711865 & -8.1 & 24.250393 \\
\hline $\mathrm{O}$ & -0.47 & 5.0 & 0654 \\
\hline 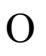 & -0.331264 & 288 & 53599 \\
\hline $\mathrm{C}$ & -0.366 & -12.6 & 99040 \\
\hline $\mathrm{O}$ & -0.429982 & -3.7 & 24.212296 \\
\hline $\mathrm{C}$ & 4.794 & 5.1 & 24.183480 \\
\hline $\mathrm{O}$ & 4.85 & 7484 & 12756 \\
\hline $\mathrm{O}$ & 4.748857 & -12. & 40486 \\
\hline $\mathrm{C}$ & 4.730866 & -3.6 & 24.199447 \\
\hline $\mathrm{O}$ & 9.978909 & 5.22 & 24.105075 \\
\hline . & 9.904612 & 14.216127 & 24.146707 \\
\hline $\mathrm{O}$ & 9.961187 & -12.723820 & 24.264747 \\
\hline $\mathrm{O}$ & 9.965342 & -3.6 & 44013 \\
\hline 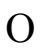 & -5.625765 & 5.258387 & 24.241923 \\
\hline $\mathrm{O}$ & -5.560916 & 14.240768 & 24.334550 \\
\hline 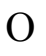 & -5.613056 & -12.759637 & 24.324138 \\
\hline $\mathrm{O}$ & -5.576140 & -3.722507 & 24.288948 \\
\hline $\mathrm{O}$ & 3.628494 & 7.409094 & 24.028492 \\
\hline 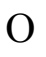 & 3.635561 & 16.364912 & 24.131188 \\
\hline $\mathrm{O}$ & 3.435316 & -10.441910 & 24.448103 \\
\hline - & 3.428330 & -1.472417 & 24.328876 \\
\hline $\mathrm{O}$ & 8.686574 & 7.492712 & 24.011278 \\
\hline $\mathrm{O}$ & 8.499111 & 16.410948 & 24.205245 \\
\hline $\mathrm{O}$ & 8.631115 & -10.411508 & 24.336052 \\
\hline $\mathrm{O}$ & 8.661666 & -1.492443 & 24.201834 \\
\hline
\end{tabular}




\begin{tabular}{|c|c|c|c|}
\hline $\mathrm{O}$ & -6.958134 & 7.464813 & 24.384694 \\
\hline$\Omega$ & -6.903269 & 16.487192 & 24.344552 \\
\hline $\mathrm{C}$ & -6.933040 & -10.437208 & 24.122454 \\
\hline C & -6.810229 & -1.471171 & 24.271180 \\
\hline $\mathrm{C}$ & -1.590154 & 7.508974 & 24.265353 \\
\hline C & -1.650831 & 16.442350 & 24.175849 \\
\hline $\mathrm{C}$ & -1.616851 & -10.447334 & 24.275030 \\
\hline 0 & -1.655750 & -1.478524 & 24.202510 \\
\hline $\mathrm{O}$ & 0.990740 & 3.003160 & 24.232707 \\
\hline$U$ & 0.993527 & 11.971645 & 24.269539 \\
\hline $\mathrm{O}$ & 1.031799 & -14.978881 & 24.310400 \\
\hline $\mathrm{O}$ & 0.946477 & -5.963921 & 24.176708 \\
\hline 0 & 6.065972 & 2.961658 & 24.195712 \\
\hline 0 & 6.070904 & 11.906646 & 24.040627 \\
\hline 0 & 6.043872 & -14.847266 & 24.396233 \\
\hline $\mathrm{O}$ & 5.939066 & -5.959435 & 24.224091 \\
\hline 0 & -9.468124 & 2.948585 & 24.250682 \\
\hline $\mathrm{O}$ & -9.430570 & 11.986405 & 23.999467 \\
\hline $\mathrm{O}$ & -9.626306 & -14.865826 & 24.395161 \\
\hline $\mathrm{O}$ & -9.487403 & -5.941518 & 24.212693 \\
\hline $\mathrm{O}$ & -4.220837 & 3.011105 & 2279 \\
\hline $\mathrm{O}$ & -4.243305 & 11.930858 & 24.170956 \\
\hline U & -4.158501 & -14.963860 & 24.232078 \\
\hline $\mathrm{O}$ & -4.194646 & -5.975074 & 24.164928 \\
\hline $\mathrm{O}$ & -0.00 & 3.22 & -26.1 \\
\hline $\mathrm{O}$ & 0.145116 & 12.127518 & -26.172111 \\
\hline $\mathrm{O}$ & 0.179421 & -14.917790 & -26.074575 \\
\hline 0 & 0.045393 & -5.662160 & -26.112024 \\
\hline $\mathrm{O}$ & 5.250118 & 3.344337 & -26.068310 \\
\hline $\mathrm{O}$ & 5.201745 & 12.213405 & -26. \\
\hline $\mathrm{O}$ & 5.194923 & -14.576503 & 6781 \\
\hline $\mathrm{O}$ & 5.318602 & -5.745226 & -26.138472 \\
\hline $\mathrm{O}$ & 10.325521 & 3.155783 & -26.176034 \\
\hline $\mathrm{O}$ & -10.342682 & 12.308079 & -26.100004 \\
\hline $\mathrm{O}$ & -10.133646 & -14.804201 & -26.098116 \\
\hline $\mathrm{O}$ & 10.298234 & -5.690089 & -26.127699 \\
\hline $\mathrm{O}$ & -5.185186 & 3.266763 & -26.039511 \\
\hline $\mathrm{O}$ & -5.143621 & 12.064135 & -26.204805 \\
\hline $\mathrm{O}$ & -4.929179 & -14.853669 & -26.065436 \\
\hline $\mathrm{O}$ & -5.112197 & -5.756794 & -26.178839 \\
\hline $\mathrm{O}$ & 2.582640 & 1.468717 & -26.097422 \\
\hline $\mathrm{O}$ & 2.408763 & 10.652379 & -26.008506 \\
\hline $\mathrm{O}$ & 2.535758 & -16.429845 & -26.007937 \\
\hline $\mathrm{O}$ & 2.098754 & -7.450845 & -26.079785 \\
\hline $\mathrm{O}$ & 7.544002 & 1.502058 & -26.009212 \\
\hline $\mathrm{O}$ & 7.492288 & 10.493742 & -26.200915 \\
\hline $\mathrm{O}$ & 7.645199 & -16.457691 & -26.017904 \\
\hline $\mathrm{O}$ & 7.598176 & -7.254981 & -26.097794 \\
\hline $\mathrm{O}$ & -7.792801 & 1.514901 & -26.101553 \\
\hline $\mathrm{O}$ & -8.058130 & 10.719499 & -26.186471 \\
\hline $\mathrm{O}$ & -7.877588 & -16.384414 & -26.046205 \\
\hline $\mathrm{O}$ & -8.029832 & -7.442290 & -26.235027 \\
\hline
\end{tabular}




\begin{tabular}{|c|c|c|c|}
\hline $\mathrm{O}$ & -2.725270 & 1.500321 & -26 \\
\hline C & -2.689325 & 10.489407 & -26.213584 \\
\hline C & -2.729101 & -16.369728 & -26.074439 \\
\hline & -2.833203 & -7.392209 & -26.110011 \\
\hline C & 2.777536 & 7.630318 & 8308 \\
\hline C & 2.568304 & 16.710313 & -26.070874 \\
\hline & 2.580428 & -10.062577 & -25.992485 \\
\hline C & 2.813922 & -1.266878 & -26.114824 \\
\hline $\mathrm{C}$ & 7.670328 & 7.737262 & -26.1 \\
\hline $\mathrm{O}$ & 7.761986 & 16.797798 & -26.042081 \\
\hline C & 7.593179 & -10.195993 & -26.009911 \\
\hline $\mathrm{C}$ & 7.846908 & -1.219253 & -26.1 \\
\hline $\mathrm{C}$ & -7.799205 & 8.016608 & -26.1 \\
\hline $\mathrm{C}$ & -7.572078 & 16.727665 & 2940 \\
\hline $\mathrm{C}$ & -7.860626 & -10.185381 & 9036 \\
\hline $\mathrm{O}$ & -7.719158 & -1.163142 & -26.1 \\
\hline $\mathrm{O}$ & -2.529027 & 7.564585 & -26.2 \\
\hline $\mathrm{O}$ & -2.426494 & 16.729051 & 474 \\
\hline $\mathrm{O}$ & -2.603933 & -10.222986 & 3413 \\
\hline $\mathrm{C}$ & -2.525413 & -1.262483 & -26. \\
\hline $\mathrm{O}$ & -0.021419 & 6.11 & -26.2 \\
\hline 0 & -0.229 & 681 & 551 \\
\hline 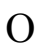 & -0.231318 & -11.717432 & 477 \\
\hline $\mathrm{C}$ & -0.306032 & -2.957635 & -26 \\
\hline $\mathrm{O}$ & 5.0652 & 6.12 & -26 . \\
\hline $\mathrm{C}$ & 5.1038 & 15.1 & -26 \\
\hline $\mathrm{O}$ & 4.842997 & -11.9 & 649 \\
\hline $\mathrm{O}$ & 4.869036 & -3.120896 & -26 \\
\hline $\mathrm{C}$ & 10.320063 & 5.9 & 3074 \\
\hline $\mathrm{O}$ & 9.990388 & 15.050795 & 0234 \\
\hline C & 10.205540 & -11.859830 & 9881 \\
\hline $\mathrm{O}$ & 10.086 & -2.924546 & 5834 \\
\hline $\mathrm{O}$ & -5.361739 & & -26 \\
\hline C & -5.212180 & 14.862560 & 7616 \\
\hline $\mathrm{O}$ & -5.430697 & -11.765215 & 4143 \\
\hline C & -5.413106 & -3.017096 & -26 \\
\hline $\mathrm{O}$ & 1.123839 & 1.61 & 26.4 \\
\hline 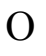 & 1.0138 & 10.6 & 683 \\
\hline C & 1.219028 & 9116 & 390 \\
\hline $\mathrm{O}$ & 0.893786 & -7.373724 & 473 \\
\hline C & 6.252132 & 1.476706 & 26.3 \\
\hline $\mathrm{O}$ & 6.192168 & 10.525366 & 1028 \\
\hline $\mathrm{O}$ & 6.252068 & -16.414045 & 3161 \\
\hline 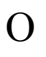 & 6.161442 & -7.121937 & 26.367716 \\
\hline $\mathrm{O}$ & -9.307987 & 1.418704 & 26.3 \\
\hline 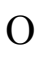 & -9.437842 & 10.731354 & 26.240702 \\
\hline $\mathrm{O}$ & -9.400240 & -16.520859 & 26.341483 \\
\hline $\mathrm{O}$ & -9.514437 & -7.289014 & 26.325892 \\
\hline $\mathrm{O}$ & -4.082239 & 1.349804 & 26.298475 \\
\hline $\mathrm{O}$ & -4.035507 & 10.494063 & 26.202596 \\
\hline $\mathrm{O}$ & -4.068693 & -16.454791 & 26.351852 \\
\hline & -4.175489 & -7.360856 & 26.28777 \\
\hline
\end{tabular}




$\begin{array}{lccc}\mathrm{O} & 0.756267 & 4.487487 & 26.298945 \\ \mathrm{O} & 0.648211 & 13.396180 & 26.328205 \\ \mathrm{O} & 0.949411 & -13.605249 & 26.480913 \\ \mathrm{O} & 0.722721 & -4.678790 & 26.326574 \\ \mathrm{O} & 5.884534 & 4.533843 & 26.273230 \\ \mathrm{O} & 6.017837 & 13.218469 & 26.313500 \\ \mathrm{O} & 5.950073 & -13.293781 & 26.477614 \\ \mathrm{O} & 5.941622 & -4.473888 & 26.302466 \\ \mathrm{O} & -9.620086 & 4.455779 & 26.307454 \\ \mathrm{O} & -9.716715 & 13.399040 & 26.285609 \\ \mathrm{O} & -9.522357 & -13.303355 & 26.426195 \\ \mathrm{O} & -9.546577 & -4.568621 & 26.348601 \\ \mathrm{O} & -4.363151 & 4.517557 & 26.287201 \\ \mathrm{O} & -4.405457 & 13.236249 & 26.383659 \\ \mathrm{O} & -4.388766 & -13.624979 & 26.415135 \\ \mathrm{O} & -4.464183 & -4.726501 & 26.278478 \\ \mathrm{O} & 3.653550 & 6.020589 & 26.206857 \\ \mathrm{O} & 3.836762 & 15.039503 & 26.319022 \\ \mathrm{O} & 3.559855 & -12.035654 & 26.479094 \\ \mathrm{O} & 3.490776 & -3.008519 & 26.414069 \\ \mathrm{O} & 8.688760 & 5.942618 & 26.130727 \\ \mathrm{O}\end{array}$




\begin{tabular}{|c|c|c|c|}
\hline 0 & 7.933713 & 4.595070 & -26.273253 \\
\hline $\mathrm{C}$ & 7.785757 & 13.308264 & -26.204906 \\
\hline & 7.930989 & -13.306955 & -26.155577 \\
\hline & 7.748274 & -4.411802 & -26.236163 \\
\hline C & -7.757094 & 4.654335 & -26.192839 \\
\hline & -7.677261 & 13.455048 & -26.460129 \\
\hline C & -7.897936 & -13.238855 & -26.047071 \\
\hline C & -7.958802 & -4.541725 & -26.188961 \\
\hline & -2.329000 & 4.615883 & -26.155439 \\
\hline C & -2.431243 & 13.460092 & -26.281070 \\
\hline C & -2.611616 & -13.497758 & -26.095687 \\
\hline $\mathrm{C}$ & -2.628005 & -4.3 & -26.350932 \\
\hline $\mathrm{O}$ & -0.111887 & 8.907986 & 4986 \\
\hline C & 0.074156 & -17.804529 & 53237 \\
\hline C & -0.120369 & -8.950707 & 60402 \\
\hline C & -0.349593 & 0.133645 & -26.048279 \\
\hline C & 5.120442 & 9.0 & -26 \\
\hline $\mathrm{O}$ & 5.114 & -17.9 & 826 \\
\hline C & 5.223540 & -8.7 & -26.206433 \\
\hline C & 5.170028 & -0.0 & -26.267453 \\
\hline $\mathrm{C}$ & -10.310661 & 8.998103 & -26.223065 \\
\hline $\mathrm{C}$ & -10.179038 & 5322 & -26.030940 \\
\hline C & -10.348737 & -8.780016 & 64280 \\
\hline $\mathrm{O}$ & -10.300 & 120 & 3367 \\
\hline C & -5.027 & & \\
\hline $\mathrm{C}$ & -5.191681 & -17.851533 & -26.251194 \\
\hline $\mathrm{C}$ & -5.0708 & -8.9 & -26.239198 \\
\hline C & $-5.108 c$ & -0.0 & 82007 \\
\hline $\mathrm{O}$ & 3.623 & 3.0 & 26. \\
\hline C & 3.3361 & 12.1 & \\
\hline C & 3.507328 & -14.9 & 26.561179 \\
\hline $\mathrm{O}$ & 3.263561 & -5.8 & 26.524357 \\
\hline C & 8.661596 & 3.1 & 26.172979 \\
\hline $\mathrm{O}$ & 8.599490 & 11.9 & 7872 \\
\hline 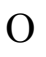 & 8.817281 & -14 & \\
\hline $\mathrm{C}$ & 8.607406 & -5.7 & 26.456092 \\
\hline $\mathrm{O}$ & -6.746201 & 2.9 & 26.552342 \\
\hline C & -7.035299 & 11.942002 & 26.303633 \\
\hline $\mathrm{O}$ & -7.1831 & -14.890815 & 26.632990 \\
\hline $\mathrm{O}$ & -7.107 & -5.8 & \\
\hline , & -1.588416 & 3.1 & 26.334787 \\
\hline $\mathrm{O}$ & -1.762217 & 12.036922 & 26.455465 \\
\hline $\mathrm{O}$ & -1.834362 & -14.891042 & 26.438083 \\
\hline $\mathrm{O}$ & -1.787435 & -5.830413 & 26.457737 \\
\hline $\mathrm{O}$ & 0.611044 & 7.689690 & 26.295251 \\
\hline 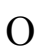 & 0.754282 & 16.540527 & 26.517797 \\
\hline $\mathrm{O}$ & 0.755945 & -10.250044 & 26.518166 \\
\hline 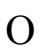 & 0.799286 & -1.178315 & 26.704089 \\
\hline $\mathrm{O}$ & 5.935178 & 7.600676 & 26.478537 \\
\hline 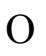 & 6.141424 & 16.618187 & 26.456229 \\
\hline $\mathrm{O}$ & 6.082627 & -10.180506 & 26.499163 \\
\hline & 5.924367 & -1.486331 & 26.465062 \\
\hline
\end{tabular}




\begin{tabular}{|c|c|c|c|}
\hline $\mathrm{O}$ & -9.338395 & 7.681904 & 26.282422 \\
\hline $\mathrm{O}$ & -9.515734 & 16.634530 & 26.639438 \\
\hline $\mathrm{O}$ & -9.446123 & -10.129394 & 26.404699 \\
\hline $\mathrm{O}$ & -9.623757 & -1.475518 & 26.350468 \\
\hline $\mathrm{O}$ & -4.341804 & 7.646506 & 26.343802 \\
\hline $\mathrm{O}$ & -4.329331 & 16.628296 & 26.562689 \\
\hline 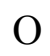 & -4.332258 & -10.373853 & 26.430892 \\
\hline $\mathrm{O}$ & -4.336490 & -1.368865 & 26.427884 \\
\hline 1 & 2.420599 & 5.760334 & -26.487177 \\
\hline $\mathrm{H}$ & 2.180121 & 14.484792 & -26.141341 \\
\hline $\mathrm{H}$ & 2.083776 & -12.651944 & -26.124854 \\
\hline $\mathrm{H}$ & 2.185184 & -3.601057 & 9173 \\
\hline $\mathrm{H}$ & 7.352053 & 5.337792 & -26.510055 \\
\hline $\mathrm{H}$ & 7.362613 & 14.222849 & -26.090011 \\
\hline $\mathrm{H}$ & 7.310 & -12.543344 & -26.332213 \\
\hline $\mathrm{H}$ & 7.255488 & -3.540263 & -26.375393 \\
\hline $\mathrm{H}$ & -8.3 & 5.4 & 8260 \\
\hline $\mathrm{H}$ & -8.112790 & 14.4 & 02383 \\
\hline $\mathrm{H}$ & $-8.2^{\prime}$ & -12.318998 & -26.209249 \\
\hline $\mathrm{H}$ & -8.5 & -3.720947 & -26.124049 \\
\hline $\mathrm{H}$ & -2.72 & 5.538526 & -26.261353 \\
\hline $\mathrm{H}$ & -3.0 & 14.274064 & -26.321821 \\
\hline $\mathrm{H}$ & -3.17 & -12.600286 & -25.977102 \\
\hline $\mathrm{H}$ & -3.1 & -3.5 & 8046 \\
\hline $\mathrm{H}$ & -0.8 & & -26 \\
\hline $\mathrm{H}$ & -0.6 & 9.78 & 1584 \\
\hline $\mathrm{H}$ & -0.3 & -16.8 & -26.251151 \\
\hline $\mathrm{H}$ & -0.7 & -8.112606 & -26.166269 \\
\hline $\mathrm{H}$ & 4.4 & 0.7 & -26 . \\
\hline $\mathrm{H}$ & 4.5 & & -26.350714 \\
\hline $\mathrm{H}$ & 4.62 & -17.0 & 75317 \\
\hline $\mathrm{H}$ & 4.6 & -7.94 & -26.438908 \\
\hline $\mathrm{H}$ & 9.9 & 0.89 & -26.479693 \\
\hline $\mathrm{H}$ & 9.8 & 9.8 & 6964 \\
\hline $\mathrm{H}$ & 9.9 & -17.2 & 85713 \\
\hline $\mathrm{H}$ & 9.8 & -7.929061 & -26.492181 \\
\hline $\mathrm{H}$ & -5.54 & 0.826973 & -26.134153 \\
\hline $\mathrm{H}$ & -5.58 & 3443 & -26.415005 \\
\hline $\mathrm{H}$ & -5.6 & -16.9 & -26.463985 \\
\hline $\mathrm{H}$ & -5.4 & -8.0 & -26.453263 \\
\hline $\mathrm{H}$ & 1.04 & 6.768665 & 26.174000 \\
\hline $\mathrm{H}$ & 1.23 & 15.696053 & 26.841856 \\
\hline $\mathrm{H}$ & 1.185284 & -11.178930 & 26.656223 \\
\hline $\mathrm{H}$ & 1.35 & -2.051617 & 26.857174 \\
\hline $\mathrm{H}$ & 6.434814 & 6.708047 & 26.662175 \\
\hline $\mathrm{H}$ & 6.818025 & 15.834041 & 26.665406 \\
\hline $\mathrm{H}$ & 6.743841 & -11.032647 & 26.632584 \\
\hline $\mathrm{H}$ & 6.486113 & -2.298285 & 26.510619 \\
\hline $\mathrm{H}$ & -8.820422 & 6.817827 & 26.458787 \\
\hline $\mathrm{H}$ & -8.99 & 15.709832 & 26.733515 \\
\hline $\mathrm{H}$ & -8.957569 & -11.066121 & 26.588732 \\
\hline 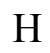 & -9.042952 & -2.282360 & 26.611797 \\
\hline
\end{tabular}




\begin{tabular}{|c|c|c|c|}
\hline $\mathrm{H}$ & -4.136231 & 6.647650 & 26.541422 \\
\hline $\mathrm{H}$ & -3.865871 & 15.672170 & 26.666489 \\
\hline $\mathrm{H}$ & -3.943685 & -11.314676 & 26.574524 \\
\hline $\mathrm{H}$ & -3.818421 & -2.220482 & 26.532200 \\
\hline $\mathrm{H}$ & 4.082521 & 2.149674 & 26.719057 \\
\hline $\mathrm{H}$ & 3.811257 & 11.396336 & 26.064953 \\
\hline $\mathrm{H}$ & 4.148193 & -15.730488 & 26.780137 \\
\hline $\mathrm{H}$ & 3.777972 & -6.799924 & 26.543686 \\
\hline $\mathrm{H}$ & 9.350094 & 2.364944 & 26.285454 \\
\hline $\mathrm{H}$ & 9.205164 & 11.132194 & 26.453573 \\
\hline $\mathrm{H}$ & 9.408900 & -15.526712 & 26.647882 \\
\hline $\mathrm{H}$ & 8.944669 & -6.668254 & 26.517120 \\
\hline $\mathrm{H}$ & -6.180612 & 2.208089 & 26.817338 \\
\hline $\mathrm{H}$ & -6.613010 & 10.969358 & 26.473650 \\
\hline $\mathrm{H}$ & -6.858512 & -15.836029 & 26.819243 \\
\hline $\mathrm{H}$ & -6.649613 & -6.780782 & 26.590227 \\
\hline $\mathrm{H}$ & -0.969339 & 2.272584 & 26.445017 \\
\hline $\mathrm{H}$ & -1.250752 & 11.156899 & 26.638899 \\
\hline $\mathrm{H}$ & -1.486222 & -15.871285 & 26.638858 \\
\hline $\mathrm{H}$ & -1.344292 & -6.761783 & 26.659143 \\
\hline $\mathrm{Na}$ & 8.824962 & 8.370014 & -19.714596 \\
\hline $\mathrm{Na}$ & 6.279527 & 17.266286 & -14.479808 \\
\hline $\mathrm{Na}$ & -2.291988 & 11.496737 & 17.172896 \\
\hline $\mathrm{Na}$ & -1.453233 & 15.527239 & -19.000113 \\
\hline $\mathrm{Na}$ & 10.150139 & 13.003814 & 6.642959 \\
\hline $\mathrm{Na}$ & -0.617160 & 1.184807 & 0.548358 \\
\hline $\mathrm{Na}$ & -7.571927 & 0.301182 & 18.176493 \\
\hline $\mathrm{Na}$ & -4.905568 & -5.514703 & 17.724482 \\
\hline $\mathrm{Na}$ & -7.493797 & -1.231970 & -19.338768 \\
\hline $\mathrm{Na}$ & -9.929177 & -6.331001 & 1.128115 \\
\hline $\mathrm{Na}$ & 9.604364 & -14.355921 & 20.272220 \\
\hline $\mathrm{Na}$ & 0.334005 & -9.719512 & -20.408868 \\
\hline $\mathrm{Na}$ & -0.943757 & -2.961349 & -18.805555 \\
\hline $\mathrm{Na}$ & -4.022779 & 2.093235 & 10.989539 \\
\hline $\mathrm{Cl}$ & 4.154699 & 8.616871 & -2.131332 \\
\hline $\mathrm{Cl}$ & -7.032106 & -14.498110 & 2.179181 \\
\hline OW & 9.140384 & 15.212657 & -9.516099 \\
\hline HW & 8.775305 & 14.363601 & -9.897964 \\
\hline HW & 8.414763 & 15.697740 & -9.028074 \\
\hline OW & 9.361129 & 4.437034 & 14.227211 \\
\hline HW & 8.615431 & 4.595797 & 14.874304 \\
\hline HW & 10.234082 & 4.650953 & 14.665608 \\
\hline OW & 0.500609 & 5.384889 & -1.259311 \\
\hline $\mathrm{HW}$ & 0.131460 & 4.845417 & -2.016081 \\
\hline HW & -0.193408 & 6.030411 & -0.940499 \\
\hline OW & 6.450121 & -1.233963 & 15.993914 \\
\hline HW & 5.527657 & -1.048991 & 16.332804 \\
\hline HW & 7.058470 & -1.416972 & 16.766196 \\
\hline OW & -4.562192 & -12.921565 & 21.216465 \\
\hline HW & -4.179504 & -12.043469 & 20.929242 \\
\hline HW & -3.849262 & -13.622101 & 21.185152 \\
\hline OW & -8.667986 & 5.534370 & -19.497784 \\
\hline
\end{tabular}




$\begin{array}{lccc}\text { HW } & -8.125512 & 5.272592 & -20.296029 \\ \text { HW } & -8.061097 & 5.686567 & -18.717706 \\ \text { OW } & -5.336444 & 14.467171 & -0.816927 \\ \text { HW } & -4.514753 & 14.748319 & -1.312689 \\ \text { HW } & -5.949867 & 15.250583 & -0.717032 \\ \text { OW } & -7.972206 & -11.173776 & 21.113504 \\ \text { HW } & -8.676446 & -10.507146 & 21.357738 \\ \text { HW } & -7.844754 & -11.816039 & 21.869318 \\ \text { OW } & 4.110802 & 8.878634 & -13.885965 \\ \text { HW } & 4.924629 & 8.348686 & -13.647551 \\ \text { HW } & 3.341932 & 8.257881 & -14.039279 \\ \text { OW } & 8.776876 & -0.600127 & 3.752062 \\ \text { HW } & 9.050519 & 0.356769 & 3.654750 \\ \text { HW } & 9.312134 & -1.165356 & 3.124355 \\ \text { OW } & -2.776979 & -14.147689 & 19.092755 \\ \text { HW } & -2.350288 & -14.339189 & 19.976645 \\ \text { HW } & -2.432231 & -14.791599 & 18.409725 \\ \text { OW } & -1.984285 & 2.289118 & -1.412590 \\ \text { HW } & -1.149247 & 2.700301 & -1.778159 \\ \text { HW } & -2.774683 & 2.832961 & -1.694561 \\ \text { OW } & -0.798191 & -10.237835 & 7.581769 \\ \text { HW } & -0.288784 & -10.885652 & 7.015344 \\ \text { HW } & -1.596140 & -10.694610 & 7.975005 \\ \text { OW } & 6.668712 & 16.317252 & -10.279792 \\ \text { HW } & 6.981598 & 16.624431 & -11.178538 \\ \text { HW } & 5.849370 & 15.754000 & -10.386682 \\ \text { OW } & -3.143465 & -5.044075 & 11.765483 \\ \text { HW } & -2.746958 & -4.591575 & -11.873586 \\ \text { HW } & -4.153221 & -3.794529 & -11.641705 \\ \text { HW } & 0.942356 & 14.158737 & 1.546270 \\ \text { HW } & 0.096278 & 14.665979 & 1.710155 \\ \text { HW } & 5.6068666 & 13.653742 & 4.052486 \\ \text { HW } & -3.075981 & -6.855286 & 12.680725 \\ \text { OW } & -2.931537 & -3.057137 & 18.617163 \\ \text { HW } & -3.531189 & -2.957636 & 17.823112 \\ \text { HW } & -3.308762 & -2.541438 & 19.386420 \\ \text { OW } & -0.850351 & -1.683660 & -20.596258 \\ \text { HW } & -0.564236 & -0.783879 & -20.925699 \\ \text { HW } & -0.468685 & -2.392604 & -21.189326 \\ \text { OW } & -6.776641 & 11.213921 & 1.658780 \\ \text { HW } & -5.955525 & 11.741676 & 1.441421 \\ \text { HW } & -7.261145 & 10.988102 & 0.813640 \\ \text { HW } & 5.335086 & 7.526973 & 21.970063 \\ \text { HW } & 4.780779 & 7.557063 & 22.801831 \\ \text { HW } & -3.741341 & -4.646950 & -11.963783 \\ \text { HW } & 17.834861 & 12.526521 \\ \text { HW } & -17.422708 & 12.442482\end{array}$




\begin{tabular}{|c|c|c|c|}
\hline HW & 6.881024 & 17.557124 & 13.481637 \\
\hline OW & -3.602324 & 16.781053 & -18.666391 \\
\hline HW & -3.865600 & 17.245610 & -19.511893 \\
\hline HW & -4.394603 & 16.301747 & -18.288821 \\
\hline OW & 1.919566 & -5.168731 & -0.625338 \\
\hline HW & 2.262318 & -5.835468 & 0.036465 \\
\hline HW & 2.098796 & -4.243834 & -0.290013 \\
\hline OW & -4.539452 & -10.747137 & -3.588491 \\
\hline HW & -4.500580 & -10.436983 & -4.538383 \\
\hline HW & -4.618185 & -11.743784 & -3.566238 \\
\hline OW & -1.046611 & -5.952849 & 1.304581 \\
\hline HW & -0.908521 & -5.004460 & 1.590045 \\
\hline HW & -1.943098 & -6.267624 & 1.616395 \\
\hline OW & 5.891514 & -17.106266 & 20.906829 \\
\hline HW & 5.741001 & 17.850150 & 21.259446 \\
\hline HW & 5.288596 & -16.465602 & 21.382265 \\
\hline OW & 2.138419 & -4.879327 & -3.474322 \\
\hline HW & 2.110985 & -5.390697 & -2.615399 \\
\hline HW & 2.485406 & -5.469897 & -4.202901 \\
\hline OW & 0.016643 & -1.160562 & 12.474759 \\
\hline HW & -0.350498 & -0.414604 & 13.030416 \\
\hline HW & 0.061934 & -0.872564 & 11.518200 \\
\hline OW & -4.365202 & 4.268082 & 5.285635 \\
\hline HW & -4.362011 & 4.942690 & 4.547465 \\
\hline HW & -5.283224 & 4.207065 & 5.677443 \\
\hline OW & -6.127538 & -14.570997 & 19.033990 \\
\hline HW & -5.493163 & -14.952970 & 19.706050 \\
\hline HW & -6.395517 & -13.649400 & 19.314786 \\
\hline OW & 2.942080 & -1.959419 & 16.661142 \\
\hline HW & 3.017053 & -1.363558 & 15.861561 \\
\hline HW & 3.279438 & -1.476723 & 17.469346 \\
\hline OW & 0.810306 & 13.757350 & 19.701728 \\
\hline HW & 1.063567 & 14.518244 & 19.104317 \\
\hline HW & 1.197514 & 12.908271 & 19.342375 \\
\hline OW & -7.648684 & -7.513930 & -20.514821 \\
\hline HW & -8.204488 & -8.323529 & -20.703584 \\
\hline HW & -8.064729 & -6.715242 & -20.949568 \\
\hline OW & -5.837489 & -10.620472 & 0.568702 \\
\hline HW & -6.315696 & -11.477779 & 0.378062 \\
\hline HW & -4.942605 & -10.631636 & 0.122542 \\
\hline OW & -6.830634 & 10.903993 & -7.923359 \\
\hline HW & -6.611591 & 10.500168 & -7.035133 \\
\hline HW & -7.345577 & 11.750281 & -7.786869 \\
\hline OW & -8.961003 & 5.004619 & 10.726638 \\
\hline HW & -8.295284 & 4.837667 & 11.453925 \\
\hline HW & -9.261349 & 5.957791 & 10.762068 \\
\hline OW & -6.530185 & -8.994121 & -2.248503 \\
\hline HW & -6.215183 & -9.195705 & -1.321067 \\
\hline HW & -6.266825 & -9.740586 & -2.859594 \\
\hline OW & -4.391073 & -17.690991 & -21.063394 \\
\hline HW & -5.165689 & -17.361873 & -20.523346 \\
\hline HW & -4.583124 & -17.559847 & -22.035977 \\
\hline
\end{tabular}




\begin{tabular}{|c|c|c|c|}
\hline OW & 8.221454 & -10.161630 & -7.778091 \\
\hline HW & 7.782049 & -10.296807 & -6.890031 \\
\hline HW & 9.174918 & -9.893195 & -7.640792 \\
\hline OW & 10.048869 & -5.932599 & 21.157841 \\
\hline HW & 9.297159 & -5.908395 & 20.498791 \\
\hline HW & 9.677968 & -5.997187 & 22.084265 \\
\hline OW & 0.606976 & -17.088047 & 21.266946 \\
\hline HW & 1.317655 & -17.655880 & 21.682278 \\
\hline HW & -0.262838 & -17.581294 & 21.278413 \\
\hline OW & 9.123265 & -12.819783 & 8.883127 \\
\hline HW & 9.223686 & -13.755894 & 9.220189 \\
\hline HW & 9.867749 & -12.255420 & 9.239832 \\
\hline OW & 6.400322 & -4.359649 & -10.838495 \\
\hline HW & 5.864807 & -4.204685 & -11.668681 \\
\hline HW & 6.960526 & -5.180253 & -10.951540 \\
\hline OW & -0.602937 & 17.738938 & 7.838624 \\
\hline HW & -0.589433 & 16.780871 & 7.552396 \\
\hline HW & -0.329457 & -17.560244 & 7.071904 \\
\hline OW & -0.229061 & 3.880325 & -3.277541 \\
\hline HW & -1.085555 & 3.837199 & -3.791893 \\
\hline HW & 0.460215 & 3.314350 & -3.729836 \\
\hline $\mathrm{OW}$ & -9.333161 & -17.544663 & 13.433553 \\
\hline HW & -8.491275 & -17.060620 & 13.194953 \\
\hline HW & -9.104988 & 17.464768 & 13.869496 \\
\hline OW & -3.240069 & 5.093851 & 14.580095 \\
\hline HW & -2.581530 & 4.899098 & 15.307006 \\
\hline $\mathrm{HW}$ & -4.154718 & 5.183661 & 14.974242 \\
\hline OW & 4.769612 & 1.963159 & -4.807233 \\
\hline HW & 5.643980 & 1.831019 & -5.274159 \\
\hline HW & 4.323849 & 1.077463 & -4.677472 \\
\hline OW & -6.063551 & 5.149734 & 18.314446 \\
\hline $\mathrm{HW}$ & -6.251620 & 5.408753 & 19.261832 \\
\hline HW & -5.241515 & 4.581268 & 18.281228 \\
\hline OW & -7.148253 & 7.875362 & 15.118877 \\
\hline HW & -6.947946 & 8.522734 & 15.854260 \\
\hline HW & -6.991895 & 8.316160 & 14.234994 \\
\hline OW & 9.595192 & 4.926020 & -13.680132 \\
\hline HW & 8.903035 & 4.610751 & -14.329380 \\
\hline HW & 9.281024 & 5.767401 & -13.240385 \\
\hline OW & 9.524303 & 7.966758 & -12.281851 \\
\hline HW & 9.877046 & 7.308418 & -11.616899 \\
\hline HW & 10.219157 & 8.662835 & -12.462560 \\
\hline OW & 5.697495 & 13.035472 & 8.808142 \\
\hline HW & 4.958900 & 13.686769 & 8.634103 \\
\hline HW & 6.049154 & 13.170050 & 9.734547 \\
\hline $\mathrm{OW}$ & -8.582794 & -0.529948 & -10.438689 \\
\hline HW & -7.953691 & -1.263589 & -10.181784 \\
\hline HW & -9.018948 & -0.162050 & -9.617457 \\
\hline $\mathrm{OW}$ & 0.687473 & 10.366461 & 3.079079 \\
\hline HW & 0.694574 & 9.370553 & 2.988982 \\
\hline HW & 1.424150 & 10.756380 & 2.526567 \\
\hline OW & -7.007762 & -15.431799 & 5.723773 \\
\hline
\end{tabular}




\begin{tabular}{|c|c|c|c|}
\hline HW & -7.199479 & -15.118404 & 4.793704 \\
\hline HW & -7.866821 & -15.657683 & 6.183114 \\
\hline OW & -10.132537 & -2.725338 & 8.949005 \\
\hline HW & 9.913132 & -3.383676 & 8.614537 \\
\hline HW & -9.274458 & -2.844154 & 8.449421 \\
\hline OW & -6.232872 & -10.203877 & -14.197306 \\
\hline HW & -5.976176 & -10.651526 & -15.053879 \\
\hline HW & -5.490024 & -9.604702 & -13.898698 \\
\hline OW & 5.114498 & -17.740730 & -16.292904 \\
\hline HW & 5.457907 & -17.714855 & -17.231733 \\
\hline HW & 4.817152 & -16.825374 & -16.021409 \\
\hline OW & -7.352748 & -10.455198 & -18.364878 \\
\hline HW & -6.649198 & -10.652776 & -19.047505 \\
\hline HW & -8.149458 & -10.051448 & -18.814588 \\
\hline OW & 5.071009 & -12.487305 & -7.417703 \\
\hline HW & 4.186903 & -12.219515 & -7.034760 \\
\hline HW & 5.106492 & -12.232831 & -8.384131 \\
\hline OW & 2.203314 & -16.215485 & 6.245549 \\
\hline HW & 2.514631 & -15.275754 & 6.104175 \\
\hline HW & 2.172766 & -16.412638 & 7.225446 \\
\hline OW & -1.613229 & 2.669868 & -13.064019 \\
\hline HW & -2.466080 & 3.068691 & -12.726994 \\
\hline HW & -0.924148 & 2.695664 & -12.339793 \\
\hline OW & 4.996357 & 0.912550 & -10.916007 \\
\hline HW & 4.122080 & 0.428106 & -10.946889 \\
\hline HW & 5.602225 & 0.552391 & -11.625379 \\
\hline OW & -3.388875 & 1.322485 & -6.794154 \\
\hline HW & -4.308163 & 1.662276 & -6.595531 \\
\hline HW & -2.755721 & 2.093616 & -6.861044 \\
\hline OW & -3.930809 & -0.302747 & 11.223317 \\
\hline HW & -3.491792 & -0.916752 & 11.879261 \\
\hline HW & -4.923535 & -0.352656 & 11.332879 \\
\hline OW & 7.020100 & 1.114044 & -3.658784 \\
\hline HW & 6.935425 & 0.149336 & -3.409447 \\
\hline HW & 6.108894 & 1.513235 & -3.760521 \\
\hline OW & 8.134008 & -2.668958 & 21.086950 \\
\hline HW & 7.551331 & -3.425996 & 20.791346 \\
\hline HW & 8.985287 & -3.031865 & 21.465926 \\
\hline OW & 3.252332 & -0.314776 & 14.675919 \\
\hline HW & 3.918738 & 0.411989 & 14.842405 \\
\hline HW & 3.496206 & -0.805384 & 13.839361 \\
\hline OW & -2.606143 & 10.911898 & -6.387267 \\
\hline HW & -1.875554 & 10.238208 & -6.498546 \\
\hline HW & -2.911303 & 10.918426 & -5.434988 \\
\hline OW & -3.987213 & 10.862260 & 7.057183 \\
\hline HW & -3.315108 & 10.609324 & 7.753099 \\
\hline HW & -4.112881 & 10.099796 & 6.422473 \\
\hline OW & 4.857411 & 10.699127 & 19.926499 \\
\hline HW & 4.033755 & 10.318164 & 20.346568 \\
\hline HW & 5.632366 & 10.091926 & 20.101856 \\
\hline OW & 8.231948 & -11.734632 & -20.018013 \\
\hline HW & 7.476846 & -12.029574 & -20.603531 \\
\hline
\end{tabular}




\begin{tabular}{|c|c|c|c|}
\hline HW & 9.007692 & -12.354360 & -20.137000 \\
\hline OW & -7.500670 & 4.804014 & -22.191740 \\
\hline HW & -6.613148 & 4.804185 & -22.652506 \\
\hline HW & -8.230618 & 4.838842 & -22.874355 \\
\hline OW & 7.209894 & 5.247311 & -5.309828 \\
\hline HW & 7.639571 & 5.971174 & -4.770013 \\
\hline HW & 6.229535 & 5.222839 & -5.114132 \\
\hline OW & 10.073658 & -8.239712 & 2.701336 \\
\hline HW & -10.102574 & -8.248100 & 3.540529 \\
\hline HW & 10.228766 & -9.088085 & 2.195168 \\
\hline OW & -0.491547 & -7.859286 & 11.168849 \\
\hline HW & -1.426239 & -7.888903 & 11.523073 \\
\hline HW & -0.061659 & -8.754353 & 11.287394 \\
\hline OW & -3.914753 & 16.856436 & -11.212769 \\
\hline HW & -4.803286 & 16.460423 & -11.444466 \\
\hline HW & -3.819194 & 17.745565 & -11.660339 \\
\hline OW & 9.791130 & -16.801172 & -13.479735 \\
\hline HW & 8.808572 & -16.707360 & -13.640293 \\
\hline HW & 10.090450 & -17.711021 & -13.767107 \\
\hline OW & 1.119747 & 4.804351 & -13.246538 \\
\hline HW & 1.870413 & 5.004159 & -12.616794 \\
\hline HW & 1.213662 & 3.870425 & -13.591449 \\
\hline OW & -4.393379 & 3.471458 & -13.259543 \\
\hline HW & -4.716181 & 2.564725 & -12.988187 \\
\hline HW & -4.943046 & 3.805607 & -14.025188 \\
\hline OW & 3.179188 & 1.836149 & -1.641424 \\
\hline HW & 3.923012 & 2.479839 & -1.821400 \\
\hline HW & 3.464661 & 1.185095 & -0.938122 \\
\hline OW & 3.554590 & 9.088504 & -11.298299 \\
\hline HW & 3.689347 & 9.099828 & -12.289113 \\
\hline HW & 4.210204 & 8.459917 & -10.879912 \\
\hline OW & -6.262970 & -0.920049 & -21.225345 \\
\hline HW & -6.641198 & -0.297734 & -21.910668 \\
\hline HW & -5.316731 & -1.140860 & -21.461724 \\
\hline OW & 6.557900 & -8.547008 & -16.881141 \\
\hline HW & 5.618409 & -8.379423 & -16.582357 \\
\hline HW & 7.100355 & -7.716138 & -16.757051 \\
\hline OW & 8.616578 & 2.989406 & 22.630660 \\
\hline HW & 7.934876 & 3.272396 & 23.305344 \\
\hline HW & 9.475740 & 2.774298 & 23.094955 \\
\hline OW & 4.110480 & -11.755879 & 13.794712 \\
\hline HW & 3.210318 & -11.506494 & 14.151807 \\
\hline HW & 4.790125 & -11.684611 & 14.524783 \\
\hline OW & 4.954386 & 11.761826 & -3.828539 \\
\hline HW & 4.265417 & 12.282166 & -4.333086 \\
\hline HW & 4.551130 & 10.908989 & -3.496798 \\
\hline OW & 8.392246 & 3.617815 & 11.798010 \\
\hline HW & 9.043735 & 3.884136 & 12.508387 \\
\hline HW & 7.483700 & 3.511264 & 12.201981 \\
\hline OW & -6.598461 & 5.531957 & -6.299287 \\
\hline HW & -6.233475 & 6.462233 & -6.262261 \\
\hline HW & -7.508985 & 5.514457 & -5.886201 \\
\hline
\end{tabular}




\begin{tabular}{|c|c|c|c|}
\hline OW & 2.789127 & -8.743053 & -6.260810 \\
\hline HW & 3.185883 & -8.616720 & -7.169998 \\
\hline HW & 2.044246 & -8.089127 & -6.128394 \\
\hline OW & 2.139224 & 4.955750 & -6.586842 \\
\hline HW & 2.065555 & 5.639484 & -5.860839 \\
\hline HW & 1.404344 & 4.284714 & -6.488552 \\
\hline OW & 6.989742 & 4.775422 & 0.717555 \\
\hline HW & 7.004672 & 5.423711 & -0.043693 \\
\hline HW & 6.066706 & 4.731302 & 1.099730 \\
\hline OW & 9.305185 & 16.499680 & 21.240866 \\
\hline HW & 8.887685 & 15.712358 & 20.787195 \\
\hline HW & 9.086550 & 16.476842 & 22.216405 \\
\hline OW & 4.933699 & -16.375268 & 13.826191 \\
\hline HW & 5.519025 & -16.065102 & 14.575318 \\
\hline HW & 4.296512 & -17.067213 & 14.165608 \\
\hline OW & 4.724235 & 1.822431 & 15.705839 \\
\hline HW & 5.716362 & 1.814771 & 15.830842 \\
\hline HW & 4.282434 & 2.089525 & 16.562269 \\
\hline OW & 9.129376 & 10.534537 & 10.207402 \\
\hline HW & 8.490352 & 9.824013 & 9.912774 \\
\hline HW & 9.378569 & 11.104268 & 9.424262 \\
\hline $\mathrm{OW}$ & -3.172437 & 6.224692 & -14.802666 \\
\hline HW & -3.829859 & 5.591787 & -14.393732 \\
\hline HW & -2.471412 & 5.706554 & -15.292663 \\
\hline OW & -2.170053 & -6.019681 & 7.673947 \\
\hline HW & -1.943271 & -6.450974 & 8.547192 \\
\hline $\mathrm{HW}$ & -2.934288 & -5.387753 & 7.802832 \\
\hline OW & 0.986652 & 11.687867 & -4.628041 \\
\hline HW & 0.516211 & 11.809672 & -5.502025 \\
\hline HW & 0.327741 & 11.770930 & -3.880420 \\
\hline OW & 2.492664 & 17.195559 & 2.627072 \\
\hline HW & 3.293039 & 17.437324 & 3.175660 \\
\hline HW & 1.861381 & 16.651855 & 3.180125 \\
\hline OW & 2.448453 & -10.431606 & -15.832352 \\
\hline HW & 1.473567 & -10.389186 & -16.050978 \\
\hline HW & 2.912963 & -9.637886 & -16.225086 \\
\hline OW & -5.742303 & 1.889157 & 12.635854 \\
\hline HW & -5.373986 & 1.966930 & 13.562296 \\
\hline HW & -6.388347 & 1.126851 & 12.596911 \\
\hline OW & 8.520116 & -9.440194 & 10.946217 \\
\hline HW & 7.929094 & -9.078915 & 10.224989 \\
\hline HW & 9.276783 & -9.947726 & 10.534068 \\
\hline OW & -2.019387 & 5.093280 & -9.293654 \\
\hline HW & -1.704980 & 5.341356 & -10.209955 \\
\hline HW & -2.918527 & 4.660041 & -9.355707 \\
\hline OW & -5.648463 & -3.238053 & 5.535333 \\
\hline HW & -5.722896 & -2.241063 & 5.557059 \\
\hline HW & -5.430243 & -3.576031 & 6.450838 \\
\hline $\mathrm{OW}$ & -8.801343 & -2.166618 & -1.164643 \\
\hline HW & -9.738367 & -1.954683 & -0.887030 \\
\hline HW & -8.276261 & -2.462865 & -0.366817 \\
\hline OW & 3.406644 & 6.343667 & -21.345920 \\
\hline
\end{tabular}




\begin{tabular}{|c|c|c|c|}
\hline HW & 2.778514 & 5.577911 & -21.207823 \\
\hline HW & 3.599104 & 6.445048 & -22.321974 \\
\hline OW & -2.514842 & 4.548858 & -22.460113 \\
\hline HW & -3.194957 & 4.143491 & -23.070950 \\
\hline HW & -1.703708 & 4.803558 & -22.986602 \\
\hline $\mathrm{OW}$ & 3.387190 & -13.749806 & 6.197360 \\
\hline HW & 4.191869 & -14.107873 & 6.670944 \\
\hline HW & 3.179685 & -12.833220 & 6.539137 \\
\hline OW & -7.292193 & -1.735864 & 9.662051 \\
\hline HW & -7.387538 & -2.190642 & 8.776565 \\
\hline HW & -6.567809 & -2.180548 & 10.188856 \\
\hline OW & 7.186597 & -15.876727 & 17.636803 \\
\hline HW & 6.477545 & -16.552823 & 17.837153 \\
\hline HW & 8.015995 & -16.102072 & 18.147995 \\
\hline OW & -5.097640 & 12.668499 & 8.722044 \\
\hline HW & -5.459935 & 13.260983 & 8.002527 \\
\hline HW & -4.593488 & 11.910852 & 8.307554 \\
\hline OW & 10.117583 & 11.424238 & 14.916857 \\
\hline HW & -10.139037 & 12.171281 & 15.393518 \\
\hline HW & -10.238024 & 10.549028 & 15.235017 \\
\hline OW & 0.909955 & -9.880833 & -10.261975 \\
\hline HW & 0.758732 & -10.850341 & -10.069140 \\
\hline HW & 0.597734 & -9.333844 & -9.485237 \\
\hline OW & 2.899613 & -4.269229 & -17.000745 \\
\hline HW & 3.550494 & -4.836710 & -17.505044 \\
\hline HW & 2.947078 & -3.328643 & -17.336968 \\
\hline $\mathrm{OW}$ & -4.054562 & 11.510455 & 11.937955 \\
\hline HW & -3.618234 & 10.725557 & 12.377902 \\
\hline HW & -4.160316 & 11.328377 & 10.960375 \\
\hline $\mathrm{OW}$ & -1.610748 & 0.572949 & 4.297867 \\
\hline HW & -1.456037 & -0.397771 & 4.114104 \\
\hline $\mathrm{HW}$ & -1.345054 & 0.780437 & 5.239332 \\
\hline OW & -0.890949 & -11.542087 & -21.464015 \\
\hline HW & -1.598874 & -12.053533 & -20.976916 \\
\hline $\mathrm{HW}$ & -1.092615 & -11.544067 & -22.443468 \\
\hline OW & -5.544014 & -4.646882 & 19.916705 \\
\hline HW & -5.798538 & -5.407738 & 20.513627 \\
\hline HW & -4.968798 & -4.004206 & 20.422763 \\
\hline $\mathrm{OW}$ & -4.311691 & 3.602646 & -9.900854 \\
\hline HW & -5.076524 & 3.101528 & -9.495996 \\
\hline HW & -4.541839 & 3.861051 & -10.839075 \\
\hline OW & -7.345176 & 6.487249 & -12.527549 \\
\hline HW & -6.756879 & 6.735611 & -11.757988 \\
\hline HW & -7.439629 & 7.270996 & -13.141406 \\
\hline OW & -7.656271 & 12.590063 & -21.602723 \\
\hline $\mathrm{HW}$ & -7.982640 & 12.465249 & -22.539689 \\
\hline HW & -6.972711 & 11.891249 & -21.391998 \\
\hline OW & -6.838053 & -6.037268 & 5.661940 \\
\hline $\mathrm{HW}$ & -7.435811 & -6.545618 & 5.042047 \\
\hline HW & -7.217928 & -6.062498 & 6.586634 \\
\hline OW & -8.490990 & -4.459907 & 21.053443 \\
\hline HW & -9.414625 & -4.843177 & 21.054904 \\
\hline
\end{tabular}




\begin{tabular}{|c|c|c|c|}
\hline HW & -7.862667 & -5.102765 & 21.491556 \\
\hline OW & 2.438696 & 4.755562 & 19.839353 \\
\hline HW & 2.760816 & 5.697608 & 19.745604 \\
\hline HW & 2.782500 & 4.369366 & 20.695304 \\
\hline OW & -1.359282 & 7.437744 & 21.458727 \\
\hline HW & -1.501603 & 7.554651 & 22.441620 \\
\hline HW & -1.836912 & 6.615888 & 21.148206 \\
\hline OW & -4.260600 & 11.622616 & -8.725835 \\
\hline HW & -5.184261 & 11.429529 & -8.394825 \\
\hline HW & -3.614031 & 11.559116 & -7.965627 \\
\hline OW & 2.818966 & -3.844678 & 14.595484 \\
\hline HW & 2.985541 & -3.363691 & 15.456242 \\
\hline HW & 3.562502 & -4.491486 & 14.425795 \\
\hline OW & -8.912858 & -0.589920 & 14.128332 \\
\hline HW & -9.399203 & -1.442705 & 13.937997 \\
\hline HW & -9.419647 & 0.177575 & 13.735756 \\
\hline OW & 6.149485 & 0.916035 & -13.752174 \\
\hline HW & 7.129927 & 0.867029 & -13.942783 \\
\hline HW & 5.786011 & 1.781972 & -14.095742 \\
\hline OW & -3.920345 & -4.120374 & 8.316510 \\
\hline HW & -3.166035 & -3.715980 & 8.833697 \\
\hline HW & -4.715761 & -4.224505 & 8.913561 \\
\hline OW & 7.354463 & -1.451452 & 18.621280 \\
\hline HW & 8.086959 & -0.771631 & 18.657237 \\
\hline HW & 7.501079 & -2.137419 & 19.333988 \\
\hline OW & -6.438469 & 0.772605 & -0.732784 \\
\hline HW & -6.618644 & 1.580374 & -1.294074 \\
\hline HW & -6.407612 & -0.038758 & -1.316512 \\
\hline OW & 8.400700 & -17.115691 & -8.251499 \\
\hline HW & 7.911532 & -16.244422 & -8.211449 \\
\hline HW & 7.746072 & -17.868799 & -8.186004 \\
\hline OW & 7.178178 & -7.844490 & 2.843879 \\
\hline HW & 6.874121 & -8.432627 & 3.593308 \\
\hline HW & 8.176361 & -7.784661 & 2.851057 \\
\hline OW & -7.021116 & -15.559911 & -17.536585 \\
\hline HW & -7.382685 & -14.629362 & -17.594424 \\
\hline HW & -6.530061 & -15.673617 & -16.672909 \\
\hline OW & 6.545225 & 6.544818 & -1.596748 \\
\hline HW & 6.991487 & 6.768081 & -2.463353 \\
\hline HW & 5.818190 & 7.207168 & -1.415878 \\
\hline OW & -5.867507 & 0.298297 & -18.019502 \\
\hline HW & -5.198049 & -0.255603 & -17.524506 \\
\hline HW & -5.452631 & 0.652511 & -18.857601 \\
\hline OW & 1.597234 & 13.620613 & 6.048552 \\
\hline HW & 1.344812 & 14.303178 & 5.362704 \\
\hline HW & 1.869459 & 12.773805 & 5.591594 \\
\hline OW & 0.223381 & -10.699280 & 10.134170 \\
\hline HW & -0.275998 & -10.616102 & 9.271789 \\
\hline HW & 0.064414 & -11.605787 & 10.525292 \\
\hline OW & 1.591854 & 3.103747 & 3.116579 \\
\hline HW & 1.579763 & 3.144865 & 2.117498 \\
\hline HW & 2.490810 & 2.794270 & 3.426583 \\
\hline
\end{tabular}




\begin{tabular}{|c|c|c|c|}
\hline OW & 0.328472 & 17.688157 & -2.965496 \\
\hline HW & 0.311283 & 16.693672 & -2.862034 \\
\hline HW & 0.706251 & 17.923630 & -3.860949 \\
\hline $\mathrm{OW}$ & 2.541107 & -5.663329 & -9.171525 \\
\hline HW & 3.171964 & -5.917149 & -9.904733 \\
\hline HW & 2.101673 & -4.793581 & -9.396109 \\
\hline OW & -3.898052 & 7.777944 & 10.177759 \\
\hline HW & -3.349378 & 6.974696 & 9.945919 \\
\hline HW & -4.868815 & 7.556630 & 10.084810 \\
\hline OW & -7.459195 & -13.411222 & -9.143842 \\
\hline HW & -8.145403 & -12.699858 & -9.295763 \\
\hline HW & -6.978876 & -13.599395 & -10.000513 \\
\hline OW & 1.695962 & -15.357317 & -16.108651 \\
\hline HW & 1.877994 & -16.053192 & -16.803363 \\
\hline HW & 2.562172 & -15.017099 & -15.742684 \\
\hline OW & 10.262094 & 3.920065 & -1.485639 \\
\hline HW & 10.339979 & 2.973570 & -1.172459 \\
\hline HW & 9.711041 & 3.950952 & -2.319537 \\
\hline OW & 0.798967 & -5.731876 & -14.869601 \\
\hline HW & 0.867991 & -4.780827 & -15.170834 \\
\hline HW & 1.647490 & -5.999302 & -14.412990 \\
\hline $\mathrm{OW}$ & -0.470907 & -7.706437 & -16.544236 \\
\hline HW & -0.687037 & -8.657645 & -16.324029 \\
\hline HW & -0.238370 & -7.214615 & -15.705167 \\
\hline OW & 5.276469 & 4.770270 & -10.239775 \\
\hline HW & 5.069944 & 4.485189 & -9.303785 \\
\hline $\mathrm{HW}$ & 5.291252 & 5.768975 & -10.288438 \\
\hline OW & -3.430523 & 9.437594 & -1.479893 \\
\hline HW & -2.436294 & 9.397448 & -1.579376 \\
\hline HW & -3.660769 & 9.801804 & -0.577486 \\
\hline OW & 6.012255 & 16.936577 & 15.412386 \\
\hline HW & 5.033642 & 16.788682 & 15.269406 \\
\hline HW & 6.178496 & 17.162273 & 16.372295 \\
\hline OW & -10.297227 & -12.582000 & -15.338089 \\
\hline HW & -9.659595 & -12.220959 & -14.657593 \\
\hline HW & 9.551138 & -12.796502 & -14.897362 \\
\hline OW & 9.015125 & 9.800372 & -17.780880 \\
\hline HW & 9.879323 & 10.125991 & -17.397300 \\
\hline HW & 8.256078 & 10.301034 & -17.364725 \\
\hline OW & -8.131787 & 7.543410 & 7.773824 \\
\hline HW & -7.200205 & 7.296796 & 7.506733 \\
\hline HW & -8.742084 & 6.768693 & 7.608440 \\
\hline OW & 5.772416 & 7.742688 & -10.325721 \\
\hline HW & 6.312461 & 7.855199 & -9.491638 \\
\hline HW & 6.385911 & 7.641683 & -11.108933 \\
\hline $\mathrm{OW}$ & 7.372184 & 1.226709 & -9.000151 \\
\hline HW & 7.106240 & 1.086780 & -8.046372 \\
\hline HW & 6.587940 & 1.048441 & -9.594442 \\
\hline $\mathrm{OW}$ & -0.802656 & 9.342716 & -1.143370 \\
\hline HW & -0.821424 & 8.618827 & -0.453709 \\
\hline HW & 0.128044 & 9.698630 & -1.227766 \\
\hline OW & -0.277761 & -4.441959 & 15.996863 \\
\hline
\end{tabular}




\begin{tabular}{|c|c|c|c|}
\hline HW & -0.314640 & -4.655162 & 16.973175 \\
\hline HW & 0.670480 & -4.483992 & 15.682103 \\
\hline $\mathrm{OW}$ & -2.081312 & -15.126360 & -1.297899 \\
\hline HW & -1.641732 & -14.577478 & -2.008882 \\
\hline HW & -2.864552 & -15.610922 & -1.687431 \\
\hline OW & -7.156434 & -14.888340 & 16.491095 \\
\hline HW & -6.566574 & -14.669881 & 17.268489 \\
\hline HW & -7.084380 & -14.164982 & 15.804392 \\
\hline OW & 8.399345 & -14.293826 & 0.195955 \\
\hline HW & 8.556621 & -14.282155 & 1.183441 \\
\hline HW & 8.626342 & -15.197538 & -0.167056 \\
\hline OW & 3.957463 & -4.408288 & -21.256476 \\
\hline HW & 4.320678 & -4.259345 & -22.176199 \\
\hline HW & 3.367373 & -5.215624 & -21.257849 \\
\hline $\mathrm{OW}$ & -10.315946 & 0.498601 & -8.457753 \\
\hline HW & 9.601154 & -0.074185 & -8.622878 \\
\hline HW & 10.242745 & 1.075872 & -7.657292 \\
\hline OW & -9.199931 & -14.919575 & -5.774927 \\
\hline HW & -8.948940 & -15.839359 & -6.076591 \\
\hline HW & -9.884212 & -14.982200 & -5.048403 \\
\hline OW & -3.920446 & -4.613907 & -3.752521 \\
\hline HW & -3.893723 & -3.845162 & -3.113523 \\
\hline HW & -4.709235 & -5.192505 & -3.545070 \\
\hline OW & 0.123601 & 11.466514 & 16.670827 \\
\hline HW & 0.471207 & 11.638428 & 15.749081 \\
\hline HW & 0.890649 & 11.302680 & 17.291147 \\
\hline $\mathrm{OW}$ & 9.075396 & -16.945481 & -0.234624 \\
\hline HW & 9.079016 & -17.168185 & 0.740255 \\
\hline HW & 9.271906 & -17.769792 & -0.765563 \\
\hline OW & -1.352188 & -15.619883 & 1.980036 \\
\hline $\mathrm{HW}$ & -1.435338 & -16.211934 & 1.178436 \\
\hline HW & -0.894595 & -14.769503 & 1.720301 \\
\hline OW & 0.709613 & 17.843815 & 10.431008 \\
\hline $\mathrm{HW}$ & -0.047478 & 17.679850 & 9.798609 \\
\hline HW & 1.043855 & 16.970111 & 10.784453 \\
\hline $\mathrm{OW}$ & 4.926501 & -2.839125 & -19.223975 \\
\hline HW & 4.708649 & -3.614721 & -19.816420 \\
\hline HW & 5.248006 & -2.073778 & -19.781541 \\
\hline $\mathrm{OW}$ & 9.290876 & -6.459820 & -20.769782 \\
\hline HW & 8.337520 & -6.185015 & -20.894659 \\
\hline HW & 9.352481 & -7.457918 & -20.767388 \\
\hline OW & -0.396761 & 11.835748 & 13.869204 \\
\hline HW & -0.337619 & 12.818424 & 14.044849 \\
\hline $\mathrm{HW}$ & -1.300160 & 11.502662 & 14.139248 \\
\hline $\mathrm{OW}$ & -5.780081 & 15.814862 & -8.175291 \\
\hline HW & -5.885148 & 16.805913 & -8.092955 \\
\hline HW & -6.530233 & 15.444525 & -8.723125 \\
\hline $\mathrm{OW}$ & -4.592608 & 13.439757 & -10.969899 \\
\hline $\mathrm{HW}$ & -5.390529 & 13.030960 & -11.412852 \\
\hline HW & -4.149321 & 12.758556 & -10.387266 \\
\hline OW & -0.015574 & -4.903221 & -17.582338 \\
\hline HW & 0.848385 & -4.881992 & -17.079223 \\
\hline
\end{tabular}




\begin{tabular}{|c|c|c|c|}
\hline HW & -0.641716 & -5.546994 & -17.142448 \\
\hline OW & -4.866877 & -7.764861 & -16.439906 \\
\hline HW & -4.836584 & -7.900981 & -15.449676 \\
\hline HW & -5.792051 & -7.946484 & -16.773171 \\
\hline OW & -6.691516 & 4.919137 & 9.015565 \\
\hline HW & -7.561239 & 4.779532 & 9.488947 \\
\hline HW & -6.264209 & 4.033194 & 8.835252 \\
\hline OW & 10.355272 & -9.315760 & 6.087607 \\
\hline HW & 10.300249 & -8.890123 & 6.990827 \\
\hline HW & 9.449160 & -9.640393 & 5.816361 \\
\hline OW & 8.402619 & 0.349181 & -18.840070 \\
\hline HW & 8.818332 & 0.798578 & -19.630782 \\
\hline HW & 8.869896 & -0.517513 & -18.665447 \\
\hline OW & 4.355875 & -16.050334 & 11.275922 \\
\hline HW & 4.315716 & -16.148798 & 12.270252 \\
\hline HW & 5.093564 & -15.420458 & 11.032876 \\
\hline OW & -4.621800 & 6.632986 & -10.833294 \\
\hline HW & -3.707916 & 6.306550 & -10.591933 \\
\hline HW & -4.580431 & 7.607785 & -11.052510 \\
\hline OW & -0.219693 & 1.759857 & -17.774448 \\
\hline HW & 0.086134 & 2.621092 & -17.368562 \\
\hline HW & -0.836824 & 1.951635 & -18.537580 \\
\hline OW & 0.210912 & -4.294559 & 21.489631 \\
\hline HW & -0.112282 & -4.330300 & 22.435288 \\
\hline HW & 0.568097 & -3.381295 & 21.293755 \\
\hline OW & -4.376883 & 1.176397 & -20.085745 \\
\hline $\mathrm{HW}$ & -3.474406 & 1.494652 & -20.376000 \\
\hline HW & -5.083531 & 1.721559 & -20.536797 \\
\hline $\mathrm{OW}$ & 6.299530 & 12.430877 & 21.241232 \\
\hline HW & 5.620472 & 11.941003 & 20.694509 \\
\hline HW & 6.357043 & 12.017406 & 22.149931 \\
\hline $\mathrm{OW}$ & -8.495230 & 4.391034 & 17.488660 \\
\hline HW & -9.077257 & 5.076705 & 17.925809 \\
\hline HW & -7.549752 & 4.515642 & 17.789566 \\
\hline $\mathrm{OW}$ & -3.943687 & 17.197379 & 19.667325 \\
\hline HW & -4.205503 & -17.861414 & 20.174347 \\
\hline HW & -4.485219 & 16.420501 & 19.988578 \\
\hline OW & -2.639567 & -14.601141 & -16.373902 \\
\hline HW & -2.831840 & -15.313566 & -17.048795 \\
\hline HW & -3.323910 & -14.638434 & -15.645695 \\
\hline $\mathrm{OW}$ & -1.082798 & 4.800082 & 3.225614 \\
\hline HW & -0.396878 & 4.142724 & 3.537694 \\
\hline HW & -1.157431 & 5.544347 & 3.889315 \\
\hline OW & -1.728376 & 2.775199 & -20.571895 \\
\hline HW & -2.096978 & 3.312441 & -21.330515 \\
\hline $\mathrm{HW}$ & -2.072814 & 3.139967 & -19.706847 \\
\hline OW & 5.294267 & -9.156915 & 5.715899 \\
\hline HW & 4.557762 & -9.403766 & 5.086118 \\
\hline $\mathrm{HW}$ & 4.933756 & -9.107376 & 6.647338 \\
\hline OW & 0.590906 & -12.368953 & -1.644635 \\
\hline HW & 0.446231 & -11.960765 & -2.545996 \\
\hline HW & 1.049193 & -13.251706 & -1.748166 \\
\hline
\end{tabular}




\begin{tabular}{|c|c|c|c|}
\hline OW & 0.585091 & 0.858850 & -1.790683 \\
\hline HW & 0.538307 & 0.280062 & -2.604818 \\
\hline HW & 1.355570 & 1.490744 & -1.874778 \\
\hline OW & -8.501932 & 14.708209 & 17.293842 \\
\hline HW & -8.996526 & 13.926495 & 17.673710 \\
\hline HW & -8.945236 & 15.556219 & 17.584290 \\
\hline OW & -7.082818 & -3.850805 & 15.635961 \\
\hline HW & -7.398416 & -4.535748 & 14.979263 \\
\hline HW & -7.215964 & -4.194196 & 16.565668 \\
\hline OW & 1.079316 & 1.479729 & 15.431315 \\
\hline HW & 1.592169 & 2.305742 & 15.665163 \\
\hline HW & 1.710776 & 0.776850 & 15.103872 \\
\hline $\mathrm{OW}$ & 8.353448 & -9.371693 & -11.991410 \\
\hline HW & 9.213222 & -8.885704 & -12.148268 \\
\hline HW & 8.548033 & -10.315072 & -11.722760 \\
\hline OW & 5.274060 & -15.017975 & 7.814488 \\
\hline HW & 5.105382 & -14.746970 & 8.762172 \\
\hline HW & 5.675602 & -15.933631 & 7.796091 \\
\hline OW & 6.919812 & 7.701314 & -13.340978 \\
\hline HW & 6.807290 & 6.790525 & -13.738223 \\
\hline HW & 7.837863 & 7.784816 & -12.953410 \\
\hline OW & 9.987172 & -10.839673 & -17.682304 \\
\hline HW & 9.427335 & -11.316130 & -18.360221 \\
\hline HW & 10.268038 & -11.483210 & -16.970283 \\
\hline OW & -9.049622 & -5.359877 & -21.427007 \\
\hline HW & -9.961917 & -5.661707 & -21.150209 \\
\hline HW & -8.956648 & -5.446239 & -22.418923 \\
\hline OW & -5.902407 & 13.907319 & 17.582769 \\
\hline HW & -5.468192 & 14.153702 & 16.716310 \\
\hline HW & -6.865993 & 14.173778 & 17.560333 \\
\hline OW & -2.873498 & -13.092060 & 10.604639 \\
\hline HW & -1.877099 & -13.168481 & 10.641371 \\
\hline HW & -3.285529 & -13.922882 & 10.978759 \\
\hline $\mathrm{OW}$ & -4.388517 & 8.985988 & 19.847427 \\
\hline HW & -4.711473 & 9.814613 & 20.304681 \\
\hline HW & -4.526021 & 8.198567 & 20.448310 \\
\hline OW & 2.682374 & 11.064079 & -14.071381 \\
\hline HW & 2.537519 & 11.235249 & -15.045916 \\
\hline HW & 3.426911 & 10.406632 & -13.955502 \\
\hline OW & 1.091065 & -8.322995 & 14.870665 \\
\hline HW & 1.507892 & -8.036433 & 15.733300 \\
\hline HW & 1.264036 & -9.296978 & 14.724247 \\
\hline OW & -1.654912 & -11.893046 & 15.669240 \\
\hline HW & -1.064715 & -12.684211 & 15.508852 \\
\hline HW & -2.429449 & -12.165290 & 16.240184 \\
\hline OW & 3.455408 & -14.481432 & -4.406815 \\
\hline HW & 4.143368 & -14.819638 & -5.048943 \\
\hline HW & 3.781847 & -13.636560 & -3.982990 \\
\hline OW & 0.699860 & -1.468522 & -17.830098 \\
\hline HW & 0.342346 & -0.553940 & -17.641092 \\
\hline HW & 1.699521 & -1.443999 & -17.821364 \\
\hline $\mathrm{OW}$ & -3.971052 & -3.659775 & 1.226216 \\
\hline
\end{tabular}




\begin{tabular}{|c|c|c|c|}
\hline HW & -4.504856 & -2.833743 & 1.407115 \\
\hline HW & -3.640092 & -4.038675 & 2.090450 \\
\hline OW & 9.410199 & 1.396089 & -21.574867 \\
\hline HW & 9.596723 & 2.266178 & -21.118627 \\
\hline HW & 9.345694 & 1.544302 & -22.561716 \\
\hline $\mathrm{OW}$ & 6.994693 & -6.729115 & 6.164872 \\
\hline HW & 7.989480 & -6.719580 & 6.063346 \\
\hline HW & 6.684866 & -7.666486 & 6.324068 \\
\hline OW & -9.949551 & 1.531461 & 12.599392 \\
\hline HW & 10.241617 & 1.627571 & 11.756125 \\
\hline HW & -9.368637 & 2.336128 & 12.722063 \\
\hline OW & 4.475826 & -16.630511 & -21.226798 \\
\hline HW & 4.594786 & -16.407709 & -22.194376 \\
\hline HW & 4.231721 & -15.802685 & -20.721706 \\
\hline $\mathrm{OW}$ & 2.554622 & 11.215132 & 8.855295 \\
\hline HW & 3.239181 & 10.869140 & 9.496909 \\
\hline HW & 2.774346 & 12.159517 & 8.610635 \\
\hline $\mathrm{OW}$ & 3.734539 & -15.003706 & 22.317370 \\
\hline HW & 3.076124 & -15.416737 & 22.946572 \\
\hline HW & 4.381143 & -14.441701 & 22.833175 \\
\hline OW & 4.923848 & -4.226120 & -2.915357 \\
\hline HW & 5.360557 & -4.702488 & -3.678481 \\
\hline HW & 3.930636 & -4.312958 & -2.992756 \\
\hline OW & -1.307583 & 6.892761 & 0.225441 \\
\hline HW & -1.261659 & 6.048833 & 0.759928 \\
\hline HW & -2.099574 & 7.429221 & 0.516922 \\
\hline $\mathrm{OW}$ & -4.489671 & -16.588617 & 15.460114 \\
\hline HW & -5.421052 & -16.917466 & 15.616278 \\
\hline HW & -3.860717 & -17.064417 & 16.074956 \\
\hline $\mathrm{OW}$ & 7.627958 & -13.896289 & 12.662862 \\
\hline HW & 6.933939 & -14.131454 & 13.343329 \\
\hline HW & 8.507516 & -14.286358 & 12.935301 \\
\hline OW & -7.801319 & 14.575348 & -5.096767 \\
\hline HW & -8.354329 & 15.204703 & -5.642745 \\
\hline HW & -6.934087 & 14.402643 & -5.563760 \\
\hline $\mathrm{OW}$ & 6.537791 & -3.089786 & -0.953701 \\
\hline HW & 6.622184 & -2.106539 & -1.115267 \\
\hline HW & 5.846793 & -3.470444 & -1.568210 \\
\hline OW & 2.982453 & -9.234484 & 1.197313 \\
\hline HW & 2.091251 & -9.130224 & 1.638776 \\
\hline HW & 2.904683 & -9.880346 & 0.437830 \\
\hline OW & 10.195521 & -5.808532 & 10.957338 \\
\hline HW & 9.922911 & -6.227814 & 11.823298 \\
\hline HW & 9.457855 & -5.221007 & 10.624678 \\
\hline $\mathrm{OW}$ & -8.785155 & -0.919978 & 3.361412 \\
\hline HW & -8.446547 & -0.337352 & 2.622567 \\
\hline HW & -8.804055 & -0.398949 & 4.214742 \\
\hline OW & -1.196123 & 13.179917 & -14.016157 \\
\hline HW & -0.623511 & 12.952251 & -13.228576 \\
\hline HW & -1.971698 & 13.734756 & -13.715097 \\
\hline OW & 0.123100 & -13.690493 & -17.683253 \\
\hline $\mathrm{HW}$ & -0.177791 & -14.424450 & -18.292163 \\
\hline
\end{tabular}




\begin{tabular}{|c|c|c|c|}
\hline HW & 0.599838 & -14.083951 & -16.897179 \\
\hline OW & 5.725469 & -6.419758 & 1.148150 \\
\hline HW & 6.309810 & -6.904343 & 1.799090 \\
\hline HW & 5.904465 & -6.757413 & 0.224056 \\
\hline OW & 8.856310 & -14.607542 & -10.174895 \\
\hline HW & 9.564785 & -15.128999 & -10.650442 \\
\hline HW & 9.132385 & -14.459285 & -9.225262 \\
\hline OW & 4.641142 & 0.696935 & -21.805598 \\
\hline HW & 3.838964 & 0.099934 & -21.795605 \\
\hline HW & 4.773442 & 1.062053 & -22.727111 \\
\hline OW & 10.107204 & 3.373953 & -20.085105 \\
\hline HW & 9.260886 & 3.732484 & -19.691149 \\
\hline HW & -9.862113 & 4.006400 & -19.894089 \\
\hline OW & 0.732561 & 15.329308 & 11.293234 \\
\hline HW & 1.708643 & 15.124857 & 11.367156 \\
\hline HW & 0.240124 & 14.506274 & 11.010180 \\
\hline OW & 1.487370 & 2.266590 & -4.747870 \\
\hline HW & 2.406893 & 1.934742 & -4.537260 \\
\hline HW & 0.855736 & 1.492598 & -4.792307 \\
\hline OW & -2.467028 & -4.413885 & -19.620857 \\
\hline HW & -2.813926 & -5.230515 & -19.159580 \\
\hline HW & -3.102076 & -4.145013 & -20.345026 \\
\hline OW & -0.590566 & -15.326133 & -5.146289 \\
\hline HW & -1.065972 & -16.159501 & -4.864356 \\
\hline HW & -0.334028 & -15.398583 & -6.110104 \\
\hline OW & -3.622913 & -4.043823 & 3.856487 \\
\hline HW & -3.253462 & -4.946937 & 4.075327 \\
\hline HW & -4.445544 & -3.879389 & 4.400766 \\
\hline OW & -1.305086 & 1.005270 & 13.611560 \\
\hline HW & -2.133706 & 1.048412 & 14.169707 \\
\hline HW & -0.654695 & 1.696495 & 13.926518 \\
\hline OW & 1.253104 & -17.347858 & 13.278674 \\
\hline HW & 0.771376 & -16.652698 & 13.812239 \\
\hline HW & 1.111876 & -17.176784 & 12.303590 \\
\hline OW & 10.207485 & -5.522189 & -7.062696 \\
\hline HW & -9.795646 & -5.709283 & -6.391060 \\
\hline HW & 9.336105 & -5.881940 & -6.729115 \\
\hline OW & -6.232335 & -1.350313 & -2.303263 \\
\hline HW & -5.478392 & -1.940376 & -2.014478 \\
\hline HW & -7.098850 & -1.835759 & -2.187100 \\
\hline OW & -2.793300 & 3.592984 & -18.346625 \\
\hline HW & -3.787575 & 3.527867 & -18.431343 \\
\hline HW & -2.550457 & 3.760604 & -17.391151 \\
\hline OW & -0.275253 & -0.989066 & -8.171870 \\
\hline HW & -0.302061 & -1.122600 & -7.181189 \\
\hline HW & -0.797611 & -1.714409 & -8.620222 \\
\hline OW & 6.827948 & 13.332661 & -18.450217 \\
\hline HW & 7.060686 & 12.815740 & -19.274004 \\
\hline HW & 7.137910 & 12.830476 & -17.642917 \\
\hline OW & -6.595043 & -17.266228 & 18.583948 \\
\hline HW & -6.360200 & -17.688835 & 19.459306 \\
\hline HW & -6.512946 & -16.272576 & 18.660867 \\
\hline
\end{tabular}




\begin{tabular}{|c|c|c|c|}
\hline OW & 3.527871 & 11.530861 & 15.240223 \\
\hline HW & 4.089749 & 11.223778 & 14.472113 \\
\hline HW & 4.120237 & 11.806841 & 15.997150 \\
\hline OW & 1.036556 & 13.688556 & -15.981088 \\
\hline HW & 0.444541 & 13.009803 & -15.546563 \\
\hline HW & 0.664677 & 14.603680 & -15.825351 \\
\hline OW & 5.972659 & 17.595316 & 18.220579 \\
\hline HW & 6.017400 & 17.899997 & 19.171982 \\
\hline HW & 5.220916 & 16.944528 & 18.114003 \\
\hline OW & -9.975459 & 16.861054 & 11.037080 \\
\hline HW & -9.784566 & 17.368788 & 11.877179 \\
\hline HW & 9.757260 & 16.835374 & 10.880182 \\
\hline OW & -4.927734 & -3.075803 & 16.882403 \\
\hline HW & -5.587455 & -3.395675 & 16.202367 \\
\hline HW & -4.526317 & -2.212337 & 16.576965 \\
\hline OW & 0.337834 & -7.525244 & 18.859246 \\
\hline HW & 1.033910 & -7.875383 & 19.486048 \\
\hline HW & 0.756820 & -6.875176 & 18.225321 \\
\hline OW & -5.916445 & -11.918805 & 8.092475 \\
\hline HW & -6.904773 & -11.784830 & 8.164990 \\
\hline HW & -5.618864 & -11.707520 & 7.161452 \\
\hline $\mathrm{OW}$ & 6.473862 & -6.134696 & -14.337155 \\
\hline HW & 7.334174 & -5.689097 & -14.089557 \\
\hline HW & 6.554048 & -7.120285 & -14.188207 \\
\hline OW & 8.631139 & 10.359347 & 21.179185 \\
\hline HW & 8.225765 & 10.197160 & 22.078833 \\
\hline HW & 8.791349 & 11.338898 & 21.057473 \\
\hline OW & -0.315567 & 8.150841 & 14.919250 \\
\hline HW & -0.545850 & 7.693043 & 14.060535 \\
\hline HW & -0.253679 & 7.473224 & 15.652057 \\
\hline OW & -8.716770 & -1.230601 & -17.050198 \\
\hline $\mathrm{HW}$ & -8.656314 & -1.009748 & -16.076767 \\
\hline HW & -9.593120 & -1.674369 & -17.237499 \\
\hline OW & 1.916835 & -6.477067 & -21.072183 \\
\hline HW & 1.193109 & -6.830945 & -20.479737 \\
\hline HW & 1.561436 & -6.361106 & -21.999676 \\
\hline OW & -5.973280 & 8.793455 & 12.592040 \\
\hline HW & -5.026494 & 9.019639 & 12.821034 \\
\hline HW & -5.990773 & 7.976192 & 12.016041 \\
\hline OW & 3.905260 & -1.065316 & 6.177151 \\
\hline HW & 3.718778 & -2.018512 & 6.415145 \\
\hline HW & 4.881138 & -0.878394 & 6.289945 \\
\hline OW & -6.460193 & 2.159147 & -9.077184 \\
\hline HW & -7.389648 & 2.482336 & -9.255124 \\
\hline HW & -6.498225 & 1.280993 & -8.600320 \\
\hline $\mathrm{OW}$ & -4.384385 & -16.392587 & -2.721342 \\
\hline HW & -3.706893 & -16.912791 & -3.241336 \\
\hline HW & -5.173811 & -16.974700 & -2.526532 \\
\hline $\mathrm{OW}$ & 5.372964 & -8.865874 & 15.048319 \\
\hline HW & 5.732753 & -8.969676 & 15.975560 \\
\hline HW & 6.107705 & -9.016464 & 14.386898 \\
\hline OW & -8.830204 & -0.871628 & -3.949803 \\
\hline
\end{tabular}




\begin{tabular}{|c|c|c|c|}
\hline HW & -9.051024 & -0.710929 & -2.987819 \\
\hline HW & -8.381093 & -0.062325 & -4.328390 \\
\hline OW & 8.327628 & -14.852016 & 22.395131 \\
\hline HW & 7.918150 & -15.715160 & 22.690614 \\
\hline HW & 7.840579 & -14.089198 & 22.820445 \\
\hline $\mathrm{OW}$ & -4.602506 & 12.877399 & 1.246531 \\
\hline HW & -5.085590 & 13.450380 & 0.584472 \\
\hline HW & -4.349063 & 13.428208 & 2.041751 \\
\hline OW & -0.472889 & -2.253670 & -0.560206 \\
\hline HW & -0.940095 & -3.100483 & -0.814423 \\
\hline HW & -1.119447 & -1.638966 & -0.108428 \\
\hline OW & 1.421026 & -10.830400 & 22.081284 \\
\hline HW & 2.170293 & -11.006414 & 22.719735 \\
\hline HW & 0.582479 & -10.646302 & 22.594067 \\
\hline OW & 3.190456 & -16.334191 & -7.411497 \\
\hline HW & 4.056881 & -16.060279 & -6.994028 \\
\hline HW & 2.632805 & -15.522479 & -7.585132 \\
\hline OW & 8.751717 & 12.952010 & -4.919107 \\
\hline HW & 8.382758 & 13.834575 & -5.210568 \\
\hline HW & 8.914031 & 12.968023 & -3.932498 \\
\hline OW & 8.313798 & 17.774741 & -15.938158 \\
\hline HW & 9.085338 & -17.506884 & -16.154201 \\
\hline HW & 8.372063 & 16.943162 & -16.490500 \\
\hline OW & -8.748572 & -9.892646 & -20.928633 \\
\hline HW & -8.411546 & -10.817876 & -21.102886 \\
\hline HW & -9.736642 & -9.863821 & -21.079917 \\
\hline $\mathrm{OW}$ & 0.691780 & 15.296515 & 21.898518 \\
\hline HW & 0.351365 & 14.972154 & 22.781076 \\
\hline HW & 0.761513 & 14.526225 & 21.264648 \\
\hline OW & 2.829874 & 16.648266 & 21.057199 \\
\hline HW & 3.716763 & 16.269221 & 21.321304 \\
\hline HW & 2.098884 & 16.104571 & 21.469568 \\
\hline OW & 8.173897 & 12.523062 & -20.553002 \\
\hline HW & 8.018935 & 11.552782 & -20.738865 \\
\hline HW & 9.068105 & 12.641054 & -20.121180 \\
\hline OW & -3.265323 & 13.915378 & 20.772469 \\
\hline HW & -2.772484 & 13.145063 & 21.177098 \\
\hline HW & -2.820693 & 14.183645 & 19.917872 \\
\hline $\mathrm{OW}$ & 2.154876 & -7.611672 & 5.061566 \\
\hline HW & 1.742224 & -7.788380 & 5.955150 \\
\hline HW & 2.908658 & -8.251126 & 4.910198 \\
\hline OW & -0.737178 & -10.589936 & -12.844799 \\
\hline HW & 0.050261 & -10.692484 & -12.236997 \\
\hline HW & -1.160380 & -9.696509 & -12.694171 \\
\hline OW & -3.418705 & 14.105780 & 3.583476 \\
\hline HW & -3.227572 & 15.083303 & 3.672448 \\
\hline HW & -2.784366 & 13.588485 & 4.157950 \\
\hline OW & 7.954206 & -1.771928 & 11.087512 \\
\hline $\mathrm{HW}$ & 7.630297 & -0.909047 & 10.699543 \\
\hline HW & 7.438572 & -1.977816 & 11.919216 \\
\hline OW & -1.498009 & -12.272719 & -8.548861 \\
\hline HW & -2.051393 & -12.709122 & -7.839412 \\
\hline
\end{tabular}




\begin{tabular}{|c|c|c|c|}
\hline HW & -2.078993 & -11.681341 & -9.108085 \\
\hline OW & -2.677943 & -2.112587 & 12.464920 \\
\hline HW & -1.689578 & -1.986434 & 12.549883 \\
\hline HW & -2.990590 & -2.768169 & 13.152281 \\
\hline OW & 7.127342 & -7.853815 & 9.129190 \\
\hline HW & 7.128146 & -7.138293 & 9.827778 \\
\hline HW & 6.190333 & -8.017125 & 8.820412 \\
\hline OW & 7.108531 & -2.188260 & -15.729164 \\
\hline HW & 6.679171 & -2.913961 & -16.266758 \\
\hline HW & 6.472796 & -1.422024 & -15.635760 \\
\hline OW & -5.992921 & 8.448758 & 2.557326 \\
\hline HW & -6.031818 & 7.904652 & 1.719211 \\
\hline HW & -6.494999 & 9.303183 & 2.423627 \\
\hline OW & -8.130546 & 17.850350 & -10.894193 \\
\hline HW & -7.688488 & -17.539831 & -10.142753 \\
\hline HW & -9.122379 & -17.923504 & -10.823472 \\
\hline OW & 0.480578 & -1.718488 & 6.477536 \\
\hline HW & 0.460728 & -2.491689 & 7.111386 \\
\hline HW & 1.063506 & -1.942615 & 5.696535 \\
\hline OW & 2.088857 & 8.981771 & -7.695869 \\
\hline HW & 2.750079 & 9.419023 & -8.305456 \\
\hline HW & 2.468549 & 8.933256 & -6.772029 \\
\hline OW & 1.037881 & -11.207272 & 3.015823 \\
\hline HW & 0.335180 & -10.615782 & 3.411234 \\
\hline HW & 0.607109 & -11.881635 & 2.416097 \\
\hline OW & 2.358175 & 10.234685 & 21.007344 \\
\hline $\mathrm{HW}$ & 2.481216 & 10.189448 & 21.998714 \\
\hline HW & 1.581676 & 9.663420 & 20.741435 \\
\hline $\mathrm{OW}$ & 7.248710 & 14.859165 & 19.968972 \\
\hline HW & 6.953643 & 13.965510 & 20.307081 \\
\hline HW & 7.922222 & 14.734129 & 19.240448 \\
\hline $\mathrm{OW}$ & 7.987891 & 16.585589 & -12.738124 \\
\hline HW & 8.448947 & 15.833014 & -13.208293 \\
\hline HW & 8.479689 & 16.803045 & -11.895007 \\
\hline $\mathrm{OW}$ & 8.109580 & -0.946151 & -5.881897 \\
\hline HW & 9.039729 & -1.121150 & -5.559100 \\
\hline HW & 7.454148 & -1.360570 & -5.250497 \\
\hline OW & -4.174895 & 1.618574 & 4.712828 \\
\hline HW & -4.066815 & 2.568026 & 5.007546 \\
\hline HW & -3.274021 & 1.196709 & 4.610567 \\
\hline $\mathrm{OW}$ & 0.279008 & 1.785585 & -7.889627 \\
\hline HW & -0.055786 & 0.860486 & -8.068806 \\
\hline HW & 1.276432 & 1.798579 & -7.960169 \\
\hline OW & 9.282089 & 14.893371 & 12.843869 \\
\hline HW & 9.779023 & 14.054179 & 13.064805 \\
\hline $\mathrm{HW}$ & 9.686117 & 15.316256 & 12.032741 \\
\hline OW & 2.401848 & -8.429028 & 20.252692 \\
\hline HW & 2.436702 & -9.257298 & 20.811936 \\
\hline $\mathrm{HW}$ & 2.466763 & -7.624901 & 20.843594 \\
\hline OW & 2.508885 & 17.889783 & 18.672818 \\
\hline HW & 2.430877 & 17.376698 & 19.527603 \\
\hline HW & 2.044029 & -17.110046 & 18.768780 \\
\hline
\end{tabular}




\begin{tabular}{|c|c|c|c|}
\hline OW & 2.546175 & -11.118365 & 6.907302 \\
\hline HW & 2.764612 & -10.593164 & 7.729768 \\
\hline HW & 1.653668 & -11.555541 & 7.018247 \\
\hline OW & -3.260411 & -11.519162 & 8.449810 \\
\hline HW & -3.178172 & -12.095362 & 9.262970 \\
\hline HW & -4.227155 & -11.352829 & 8.255545 \\
\hline OW & 10.339570 & -2.618199 & -5.168192 \\
\hline HW & -9.870458 & -3.473846 & -5.256499 \\
\hline HW & -9.814935 & -1.940061 & -4.698783 \\
\hline OW & 7.784018 & 13.252998 & -13.372759 \\
\hline HW & 8.123581 & 13.938264 & -14.017047 \\
\hline HW & 8.209997 & 12.370035 & -13.570038 \\
\hline $\mathrm{OW}$ & 6.936829 & -4.735556 & 4.142405 \\
\hline HW & 6.094744 & -4.298164 & 3.826841 \\
\hline HW & 6.709587 & -5.550891 & 4.674938 \\
\hline OW & 7.868292 & 7.136699 & -8.539421 \\
\hline HW & 7.717734 & 7.445970 & -7.600441 \\
\hline HW & 7.864997 & 6.137049 & -8.565669 \\
\hline OW & 3.274868 & -1.303705 & -17.486002 \\
\hline HW & 3.868945 & -1.660627 & -18.206890 \\
\hline HW & 3.771456 & -1.296351 & -16.618046 \\
\hline OW & -9.794084 & 12.737492 & -19.643806 \\
\hline HW & -9.665252 & 13.593156 & -19.142567 \\
\hline HW & -9.130769 & 12.685390 & -20.390331 \\
\hline OW & -9.840999 & 17.008216 & 18.032558 \\
\hline HW & -9.529984 & 17.435975 & 18.881258 \\
\hline HW & -9.945998 & 17.707758 & 17.325722 \\
\hline OW & 3.236223 & 1.269390 & 4.646742 \\
\hline HW & 3.622828 & 1.133432 & 3.734573 \\
\hline HW & 3.623042 & 0.595761 & 5.276499 \\
\hline OW & 8.393341 & -9.267933 & -3.159071 \\
\hline HW & 7.673243 & -8.947117 & -3.774323 \\
\hline HW & 8.004277 & -9.447326 & -2.255496 \\
\hline $\mathrm{OW}$ & -0.897978 & -9.655441 & 3.953936 \\
\hline HW & -0.726260 & -9.034732 & 4.718941 \\
\hline HW & -1.882267 & -9.715489 & 3.787896 \\
\hline OW & 5.158471 & -3.830177 & 18.532946 \\
\hline HW & 4.266788 & -4.260080 & 18.391226 \\
\hline HW & 5.073513 & -2.840524 & 18.417319 \\
\hline OW & -3.260518 & -17.765249 & -8.736221 \\
\hline HW & -3.291319 & 17.305618 & -9.323039 \\
\hline HW & -4.174169 & -17.581504 & -8.373620 \\
\hline OW & 7.773773 & -9.694052 & -0.630793 \\
\hline HW & 8.412624 & -10.409670 & -0.348377 \\
\hline HW & 6.895457 & -9.831693 & -0.172956 \\
\hline OW & 1.617488 & 3.713969 & -20.355296 \\
\hline HW & 1.397487 & 4.006418 & -19.424666 \\
\hline HW & 0.784081 & 3.404601 & -20.813253 \\
\hline OW & 1.233984 & -3.655354 & 12.421235 \\
\hline HW & 0.801469 & -2.768353 & 12.582974 \\
\hline HW & 1.669152 & -3.972580 & 13.263848 \\
\hline $\mathrm{OW}$ & 10.059212 & -17.167115 & -21.102978 \\
\hline
\end{tabular}




\begin{tabular}{|c|c|c|c|}
\hline HW & 10.096164 & -16.812402 & -22.037223 \\
\hline HW & -9.820333 & -17.671296 & -20.904389 \\
\hline OW & 1.117019 & -12.607108 & -9.490671 \\
\hline HW & 0.215607 & -12.741811 & -9.079196 \\
\hline HW & 1.068977 & -12.803053 & -10.470108 \\
\hline OW & 3.959466 & 17.009066 & 0.205499 \\
\hline HW & 3.284462 & 17.180177 & 0.923198 \\
\hline HW & 4.389563 & 17.871888 & -0.060120 \\
\hline OW & -2.920273 & -15.483421 & -19.453729 \\
\hline HW & -2.011791 & -15.697581 & -19.094848 \\
\hline HW & -3.155219 & -16.133962 & -20.175945 \\
\hline OW & 0.239884 & 14.858482 & -2.522305 \\
\hline HW & -0.184563 & 13.954598 & -2.575576 \\
\hline HW & 1.032521 & 14.819173 & -1.913879 \\
\hline OW & 1.531952 & 10.525336 & -0.365742 \\
\hline HW & 1.930109 & 11.084903 & 0.361139 \\
\hline HW & 2.263691 & 10.086894 & -0.887591 \\
\hline OW & 6.310566 & 4.579722 & -21.467559 \\
\hline $\mathrm{HW}$ & 5.868658 & 4.784108 & -22.341025 \\
\hline HW & 5.666346 & 4.099271 & -20.872457 \\
\hline OW & 2.941932 & 6.089130 & -0.813564 \\
\hline HW & 2.037921 & 5.801500 & -1.129846 \\
\hline HW & 3.277181 & 6.831767 & -1.393304 \\
\hline OW & -4.287235 & -8.205913 & -13.768725 \\
\hline HW & -4.809119 & -7.396337 & -13.499980 \\
\hline HW & -3.538554 & -8.349814 & -13.121601 \\
\hline $\mathrm{OW}$ & -3.539521 & 4.188706 & 11.701253 \\
\hline HW & -3.355292 & 4.770461 & 12.493479 \\
\hline HW & -3.109429 & 4.584507 & 10.889858 \\
\hline $\mathrm{OW}$ & 6.182064 & 9.323695 & -19.462400 \\
\hline HW & 5.873096 & 9.315603 & -20.413438 \\
\hline $\mathrm{HW}$ & 5.437226 & 9.635714 & -18.872603 \\
\hline OW & -6.405975 & -6.845114 & -5.704082 \\
\hline HW & -5.670365 & -6.457263 & -6.259465 \\
\hline HW & -6.312355 & -7.840294 & -5.674913 \\
\hline OW & 6.049047 & -16.659626 & -2.994584 \\
\hline HW & 6.256823 & -17.607977 & -2.754879 \\
\hline HW & 6.564881 & -16.401628 & -3.811500 \\
\hline $\mathrm{OW}$ & -0.079836 & -7.692296 & -19.347079 \\
\hline HW & -0.889307 & -7.201534 & -19.669430 \\
\hline HW & -0.071621 & -7.701130 & -18.347152 \\
\hline OW & -6.997096 & 15.245743 & -21.445152 \\
\hline HW & -6.644613 & 15.537674 & -22.334271 \\
\hline HW & -7.302500 & 14.295304 & -21.503412 \\
\hline OW & 7.143910 & 8.801478 & 9.131792 \\
\hline HW & 7.143944 & 9.171958 & 8.202952 \\
\hline HW & 6.657083 & 7.928042 & 9.142241 \\
\hline OW & 8.153862 & 6.088807 & 20.963467 \\
\hline $\mathrm{HW}$ & 8.950718 & 6.643093 & 20.723076 \\
\hline HW & 8.203556 & 5.834962 & 21.929434 \\
\hline OW & -7.958564 & 0.911040 & 1.502549 \\
\hline HW & -7.606955 & 0.753932 & 0.579679 \\
\hline
\end{tabular}




\begin{tabular}{|c|c|c|c|}
\hline HW & -7.235912 & 1.302157 & 2.072462 \\
\hline OW & 10.348023 & -7.837772 & 17.093644 \\
\hline HW & -9.808357 & -8.035232 & 17.895730 \\
\hline HW & 9.815065 & -7.007545 & 17.256981 \\
\hline OW & 6.829606 & 1.058331 & -16.883115 \\
\hline HW & 6.030066 & 1.471114 & -17.319403 \\
\hline HW & 7.476291 & 0.761022 & -17.585543 \\
\hline OW & -4.289463 & -16.194427 & 1.700877 \\
\hline HW & -3.683411 & -15.415932 & 1.864109 \\
\hline HW & -5.237440 & -15.877756 & 1.668307 \\
\hline OW & -3.394416 & -10.723126 & 3.665470 \\
\hline HW & -3.539083 & -11.706008 & 3.551388 \\
\hline HW & -3.990825 & -10.223324 & 3.037383 \\
\hline OW & 1.872119 & 5.477767 & 13.015112 \\
\hline HW & 1.888808 & 4.487139 & 13.150674 \\
\hline HW & 2.485577 & 5.914600 & 13.673022 \\
\hline OW & 0.315532 & -0.477654 & -10.827371 \\
\hline HW & -0.528682 & -0.133382 & -11.238198 \\
\hline HW & 0.167242 & -0.646695 & -9.852981 \\
\hline OW & 3.763995 & -5.769659 & -14.647953 \\
\hline HW & 4.740435 & -5.814148 & -14.436797 \\
\hline HW & 3.605049 & -5.069401 & -15.343923 \\
\hline OW & 6.592311 & 16.215841 & 7.204746 \\
\hline HW & 5.907881 & 16.241735 & 6.476127 \\
\hline HW & 6.149288 & 15.981255 & 8.070021 \\
\hline OW & 2.432602 & -2.795200 & -13.235375 \\
\hline HW & 3.389896 & -3.083659 & -13.215914 \\
\hline HW & 2.008431 & -3.104791 & -14.086393 \\
\hline OW & -6.793752 & -9.538202 & -5.542705 \\
\hline HW & -6.252191 & -10.236901 & -6.010179 \\
\hline HW & -7.630259 & -9.362314 & -6.061664 \\
\hline OW & 0.910692 & 15.353018 & -19.330567 \\
\hline HW & 0.927874 & 15.129248 & -20.305058 \\
\hline HW & 1.830513 & 15.605540 & -19.030296 \\
\hline OW & -2.250344 & 2.662130 & 2.169368 \\
\hline HW & -2.301049 & 2.045669 & 2.955119 \\
\hline HW & -1.784083 & 3.505377 & 2.436830 \\
\hline OW & 4.429287 & 14.528686 & -10.671726 \\
\hline HW & 4.671794 & 13.685896 & -10.191210 \\
\hline HW & 3.843591 & 14.310567 & -11.452357 \\
\hline OW & 9.336913 & -5.643309 & -14.549971 \\
\hline HW & 10.258811 & -5.889308 & -14.849285 \\
\hline HW & 9.334966 & -4.700197 & -14.217500 \\
\hline OW & 7.650038 & 10.573818 & 12.734139 \\
\hline HW & 6.696725 & 10.323415 & 12.565338 \\
\hline HW & 7.770618 & 10.793212 & 13.702295 \\
\hline OW & -7.630931 & -4.179877 & -14.351096 \\
\hline HW & -8.181118 & -3.865711 & -13.577407 \\
\hline HW & -8.005398 & -5.039284 & -14.699226 \\
\hline OW & -1.933617 & 11.811681 & 21.928051 \\
\hline HW & -2.670440 & 11.733387 & 22.599588 \\
\hline HW & -1.162330 & 11.241812 & 22.211537 \\
\hline
\end{tabular}




\begin{tabular}{|c|c|c|c|}
\hline OW & -9.237866 & 14.627860 & -13.871975 \\
\hline HW & -9.275668 & 13.694127 & -14.227944 \\
\hline HW & -8.386364 & 14.756573 & -13.363667 \\
\hline OW & -9.164674 & 12.699270 & 21.311709 \\
\hline HW & -8.754113 & 13.607966 & 21.387290 \\
\hline HW & -9.421613 & 12.372894 & 22.221358 \\
\hline OW & -3.317454 & -0.833031 & 16.392882 \\
\hline HW & -3.245009 & -0.448096 & 17.312979 \\
\hline HW & -2.401992 & -0.953418 & 16.008909 \\
\hline OW & 3.713998 & -4.949642 & 4.227587 \\
\hline HW & 3.263408 & -4.565122 & 3.421911 \\
\hline HW & 3.372167 & -5.875128 & 4.390767 \\
\hline OW & 0.398772 & -12.844072 & 18.696187 \\
\hline HW & 0.252426 & -12.116850 & 18.025566 \\
\hline HW & 0.139742 & -12.516598 & 19.604848 \\
\hline OW & 2.026520 & 6.443563 & -9.082765 \\
\hline HW & 1.455095 & 7.262353 & -9.027475 \\
\hline HW & 2.099598 & 6.026314 & -8.176916 \\
\hline OW & -4.020109 & -11.207844 & 13.997068 \\
\hline HW & -4.225671 & -12.183434 & 14.074326 \\
\hline HW & -3.230101 & -10.987347 & 14.569142 \\
\hline $\mathrm{OW}$ & 4.618258 & -8.563429 & 8.462324 \\
\hline HW & 3.955796 & -9.247839 & 8.766838 \\
\hline HW & 4.331975 & -7.658817 & 8.778110 \\
\hline OW & -7.764560 & 11.490358 & -3.723351 \\
\hline HW & -8.518424 & 11.412100 & -4.375705 \\
\hline $\mathrm{HW}$ & -7.338576 & 12.390667 & -3.812685 \\
\hline OW & -5.316322 & 2.269071 & 9.026216 \\
\hline HW & -6.233394 & 1.889711 & 9.148957 \\
\hline HW & -4.707614 & 1.560624 & 8.669037 \\
\hline OW & 2.179655 & -1.329707 & -6.807413 \\
\hline HW & 2.936539 & -1.929770 & -7.066354 \\
\hline HW & 2.291081 & -0.441423 & -7.252986 \\
\hline OW & 6.637902 & 3.091011 & -11.743366 \\
\hline HW & 6.083864 & 3.619848 & -11.100425 \\
\hline HW & 6.120952 & 2.291733 & -12.049825 \\
\hline OW & 7.067828 & 16.403191 & -7.750985 \\
\hline HW & 6.822078 & 16.340516 & -8.718290 \\
\hline HW & 6.241078 & 16.338353 & -7.192164 \\
\hline OW & -8.247024 & -9.993264 & 8.300980 \\
\hline HW & -8.003883 & -9.837954 & 7.343503 \\
\hline HW & -7.822949 & -9.289487 & 8.870943 \\
\hline OW & -7.854431 & -14.869772 & 10.698552 \\
\hline HW & -7.288234 & -15.011218 & 9.886509 \\
\hline HW & -7.615367 & -15.547646 & 11.393776 \\
\hline OW & -2.124069 & -0.863855 & -5.866878 \\
\hline HW & -2.369428 & 0.062160 & -6.153749 \\
\hline HW & -2.922168 & -1.460742 & -5.949119 \\
\hline $\mathrm{OW}$ & 9.731583 & -11.903970 & -0.567930 \\
\hline HW & 10.343060 & -12.191064 & -1.305271 \\
\hline HW & 9.017682 & -12.592407 & -0.439869 \\
\hline OW & 4.777749 & 17.202380 & -6.330475 \\
\hline
\end{tabular}




\begin{tabular}{|c|c|c|c|}
\hline HW & 4.214459 & 16.478011 & -6.727960 \\
\hline HW & 4.607300 & 17.255141 & -5.346522 \\
\hline OW & 4.425471 & -3.081871 & -7.026860 \\
\hline HW & 4.637159 & -2.763990 & -7.951058 \\
\hline HW & 5.275832 & -3.222463 & -6.519791 \\
\hline OW & -3.001746 & -8.426065 & 20.180649 \\
\hline HW & -3.365444 & -9.342676 & 20.014672 \\
\hline HW & -2.365482 & -8.454491 & 20.951597 \\
\hline OW & -6.167750 & 5.542234 & -2.406568 \\
\hline HW & -7.042114 & 5.068389 & -2.511258 \\
\hline HW & -6.146167 & 6.339197 & -3.010210 \\
\hline OW & -9.000750 & 8.997147 & 5.235806 \\
\hline HW & -8.714889 & 8.897957 & 6.188930 \\
\hline HW & -8.474358 & 9.729858 & 4.804476 \\
\hline OW & 2.744368 & 14.169550 & 14.115148 \\
\hline HW & 3.113280 & 13.304494 & 14.455120 \\
\hline HW & 1.745754 & 14.147832 & 14.163096 \\
\hline OW & -4.256182 & 15.036142 & 15.743678 \\
\hline HW & -3.707122 & 15.834781 & 15.990068 \\
\hline HW & -4.837838 & 15.259946 & 14.961636 \\
\hline OW & -6.044675 & 10.145812 & -5.396714 \\
\hline HW & -6.609790 & 10.597019 & -4.706021 \\
\hline HW & -5.082131 & 10.209053 & -5.133066 \\
\hline OW & -6.777775 & 0.045586 & -7.455516 \\
\hline HW & -6.116739 & -0.077545 & -6.715334 \\
\hline HW & -7.278087 & -0.807949 & -7.601001 \\
\hline OW & 2.289840 & 2.898340 & 7.783157 \\
\hline HW & 2.654733 & 2.510017 & 8.629359 \\
\hline HW & 3.035516 & 3.037352 & 7.131511 \\
\hline OW & 3.558888 & 2.656035 & 18.403174 \\
\hline HW & 2.975608 & 3.307091 & 18.888881 \\
\hline HW & 4.072567 & 2.110347 & 19.065260 \\
\hline OW & 9.944125 & -8.791272 & -16.061225 \\
\hline HW & 10.218299 & -7.964161 & -16.551853 \\
\hline HW & 9.727444 & -9.512625 & -16.719023 \\
\hline OW & 7.864073 & 2.357872 & -0.082160 \\
\hline HW & 8.855833 & 2.280935 & -0.184593 \\
\hline HW & 7.650618 & 3.112444 & 0.538370 \\
\hline OW & 6.454559 & -1.012628 & -20.764920 \\
\hline HW & 5.919800 & -0.341084 & -21.277815 \\
\hline HW & 7.107374 & -0.538128 & -20.174426 \\
\hline OW & 1.926178 & -6.152876 & 17.008014 \\
\hline HW & 2.048001 & -5.322806 & 17.552204 \\
\hline HW & 2.799127 & -6.635349 & 16.936033 \\
\hline OW & 6.622949 & -5.747576 & 17.032790 \\
\hline HW & 6.076195 & -6.460262 & 17.472265 \\
\hline HW & 6.249026 & -4.849709 & 17.265206 \\
\hline OW & 5.618432 & 15.644623 & 9.734841 \\
\hline HW & 5.647968 & 14.810933 & 10.286284 \\
\hline HW & 4.784291 & 16.154305 & 9.945634 \\
\hline OW & 2.335916 & -0.374543 & -20.925108 \\
\hline HW & 2.068990 & 0.137137 & -20.108447 \\
\hline
\end{tabular}




\begin{tabular}{|c|c|c|c|}
\hline HW & 1.684608 & -0.190703 & -21.661315 \\
\hline OW & -2.422310 & 14.305975 & -4.198292 \\
\hline HW & -1.968176 & 14.362088 & -5.087457 \\
\hline HW & -3.142585 & 14.997812 & -4.147645 \\
\hline OW & 7.502834 & -10.871909 & 13.069617 \\
\hline HW & 7.438097 & -11.766927 & 12.628309 \\
\hline HW & 7.651561 & -10.167864 & 12.375209 \\
\hline OW & 3.351369 & 12.541123 & -21.156390 \\
\hline HW & 3.447288 & 12.036090 & -22.014144 \\
\hline HW & 3.239573 & 13.515060 & -21.353743 \\
\hline OW & -6.381879 & 17.711294 & 21.471969 \\
\hline HW & -7.323515 & -17.879933 & 21.298962 \\
\hline HW & -6.365458 & 16.732271 & 21.675055 \\
\hline OW & 4.885888 & 3.808249 & 9.966925 \\
\hline HW & 5.575467 & 4.176054 & 9.343066 \\
\hline HW & 4.892174 & 2.809405 & 9.919267 \\
\hline OW & -7.362255 & -5.935267 & 13.799608 \\
\hline HW & -7.975263 & -6.712497 & 13.941509 \\
\hline HW & -7.592256 & -5.482271 & 12.938275 \\
\hline OW & 4.019034 & 12.774772 & -18.552025 \\
\hline HW & 3.833192 & 12.773070 & -19.534604 \\
\hline HW & 4.964113 & 12.490313 & -18.391059 \\
\hline OW & 2.832260 & 5.863500 & 10.154985 \\
\hline HW & 3.719930 & 5.414120 & 10.054486 \\
\hline HW & 2.317070 & 5.423113 & 10.890267 \\
\hline OW & -4.626962 & -13.949531 & 13.840387 \\
\hline HW & -4.284532 & -14.205765 & 12.936459 \\
\hline HW & -4.652843 & -14.757916 & 14.428472 \\
\hline $\mathrm{OW}$ & -3.480072 & -13.252770 & -0.141684 \\
\hline HW & -2.929785 & -14.075923 & -0.281699 \\
\hline HW & -3.608309 & -12.784841 & -1.016097 \\
\hline OW & 4.313128 & 0.875907 & 20.447725 \\
\hline HW & 5.032694 & 0.465750 & 21.008078 \\
\hline HW & 3.432261 & 0.775047 & 20.910220 \\
\hline OW & -0.916600 & 6.770591 & 5.326816 \\
\hline HW & -0.134080 & 7.009114 & 5.901942 \\
\hline HW & -1.406306 & 7.603252 & 5.068236 \\
\hline OW & -1.455272 & 17.143562 & -20.653821 \\
\hline HW & -0.571784 & 17.588835 & -20.799361 \\
\hline HW & -2.137825 & 17.550973 & -21.260565 \\
\hline OW & 10.071639 & -15.066698 & 10.196711 \\
\hline HW & -9.679089 & -15.072451 & 10.442636 \\
\hline HW & 9.525208 & -15.339144 & 10.988662 \\
\hline OW & 7.586433 & -13.804691 & 3.163358 \\
\hline HW & 6.899687 & -13.108686 & 2.953697 \\
\hline HW & 7.482304 & -14.100269 & 4.112985 \\
\hline OW & 0.679153 & -7.209252 & -5.220047 \\
\hline HW & 1.103976 & -6.384629 & -5.593574 \\
\hline HW & -0.208027 & -6.976050 & -4.821891 \\
\hline OW & 6.655882 & -13.687403 & -21.299730 \\
\hline HW & 7.228953 & -14.247016 & -20.701046 \\
\hline HW & 6.739143 & -14.013815 & -22.241283 \\
\hline
\end{tabular}




\begin{tabular}{|c|c|c|c|}
\hline OW & 4.740783 & -11.179686 & 221 \\
\hline HW & 5.469009 & -10.495394 & 11.280366 \\
\hline HW & 4.579577 & -11.442598 & 12.269478 \\
\hline OW & 7.456270 & -17.204802 & 6.826344 \\
\hline HW & 8.423166 & -16.997492 & 6.975117 \\
\hline HW & 7.256940 & 17.755102 & 7.163523 \\
\hline OW & 4.498978 & 3.682753 & -7.543438 \\
\hline HW & 5.115672 & 3.894576 & -6.785270 \\
\hline HW & 3.612515 & 4.116976 & -7.383335 \\
\hline OW & -1.990765 & 0.179289 & -12.026445 \\
\hline HW & -1.741534 & 1.002138 & -12.537135 \\
\hline HW & -2.621778 & 0.422534 & -11.289794 \\
\hline OW & 9.595240 & -3.961051 & -9.150831 \\
\hline HW & 9.900742 & -4.376252 & -8.293931 \\
\hline HW & 9.174585 & -3.074110 & -8.960078 \\
\hline OW & -9.343130 & 5.494938 & -5.327276 \\
\hline HW & -9.445049 & 6.488809 & -5.370079 \\
\hline HW & -9.609252 & 5.094517 & -6.204113 \\
\hline OW & -0.288421 & -10.464538 & 17.481942 \\
\hline HW & -0.439479 & -9.476232 & 17.461124 \\
\hline HW & -0.653339 & -10.875172 & 16.646349 \\
\hline OW & -9.768803 & -13.822248 & 18.480514 \\
\hline HW & -10.219485 & -14.110159 & 17.635533 \\
\hline HW & -8.778801 & -13.803826 & 18.340668 \\
\hline OW & -5.423267 & 3.309043 & -6.753059 \\
\hline HW & -5.924889 & 2.859844 & -7.492380 \\
\hline HW & -5.909886 & 4.137328 & -6.475307 \\
\hline OW & 3.881831 & 14.827627 & -7.004265 \\
\hline HW & 3.456795 & 14.404923 & -6.203848 \\
\hline HW & 4.257489 & 14.115459 & -7.597307 \\
\hline OW & 6.591117 & -15.321361 & -11.533446 \\
\hline HW & 7.319371 & -15.045482 & -10.906121 \\
\hline HW & 5.825755 & -14.682278 & -11.457321 \\
\hline OW & -8.754984 & 9.304395 & -12.375397 \\
\hline HW & -8.094630 & 8.934284 & -13.028812 \\
\hline HW & -8.359735 & 9.288708 & -11.456956 \\
\hline OW & 10.311487 & -10.433569 & 16.586802 \\
\hline HW & -9.493177 & -10.728871 & 16.313019 \\
\hline HW & 10.286992 & -9.435620 & 16.645954 \\
\hline OW & -5.187757 & 12.779786 & 5.065363 \\
\hline HW & -4.591533 & 12.122681 & 5.526589 \\
\hline HW & -4.717405 & 13.152268 & 4.265346 \\
\hline OW & -8.874555 & -7.957636 & -2.972014 \\
\hline HW & -8.055615 & -8.394702 & -2.600112 \\
\hline HW & -9.076285 & -8.340210 & -3.873647 \\
\hline OW & 7.867303 & -2.330221 & 5.515728 \\
\hline HW & 8.331734 & -1.697335 & 4.896245 \\
\hline HW & 7.660723 & -3.179995 & 5.030746 \\
\hline OW & -3.346150 & -4.665231 & -15.508553 \\
\hline HW & -3.412792 & -5.654204 & -15.376296 \\
\hline HW & -2.905768 & -4.475224 & -16.386027 \\
\hline OW & -0.177870 & 3.017383 & -10.968770 \\
\hline
\end{tabular}




\begin{tabular}{|c|c|c|c|}
\hline HW & 0.468257 & 3.762044 & -10.801438 \\
\hline HW & -0.280237 & 2.471706 & -10.137050 \\
\hline OW & 5.460562 & -15.947989 & -6.046955 \\
\hline HW & 6.204583 & -15.508215 & -6.549976 \\
\hline HW & 5.490934 & -16.935334 & -6.202610 \\
\hline OW & -9.123667 & 15.838650 & 1.547984 \\
\hline HW & -9.821121 & 16.534820 & 1.377972 \\
\hline HW & -9.351865 & 15.007443 & 1.041012 \\
\hline OW & -2.710019 & 5.233240 & 20.796283 \\
\hline HW & -2.491124 & 4.455409 & 21.385405 \\
\hline HW & -3.548343 & 5.671463 & 21.120588 \\
\hline OW & -3.558509 & -10.414207 & -0.981119 \\
\hline HW & -2.589645 & -10.383517 & -0.735436 \\
\hline HW & -3.648026 & -10.526759 & -1.970725 \\
\hline OW & 3.182175 & -10.173542 & 4.468697 \\
\hline HW & 2.878797 & -10.674520 & 5.279242 \\
\hline HW & 2.694370 & -10.513611 & 3.664708 \\
\hline OW & -0.518469 & -12.264919 & 21.119288 \\
\hline HW & -0.894301 & -12.907919 & 21.786597 \\
\hline HW & 0.366588 & -11.929215 & 21.441743 \\
\hline OW & -6.346455 & -0.888869 & 19.789604 \\
\hline HW & -7.070073 & -1.134209 & 20.434729 \\
\hline HW & -5.618499 & -1.573351 & 19.829160 \\
\hline OW & -10.177249 & 11.091357 & -13.838038 \\
\hline HW & -9.481981 & 11.628715 & -14.315372 \\
\hline HW & -9.736841 & 10.505756 & -13.157514 \\
\hline OW & 2.728433 & -15.399662 & -10.976524 \\
\hline HW & 3.062342 & -16.295374 & -10.682916 \\
\hline HW & 3.503528 & -14.781565 & -11.107610 \\
\hline OW & 7.983044 & 14.833159 & 15.248231 \\
\hline HW & 8.521844 & 15.015321 & 14.425726 \\
\hline HW & 7.283197 & 15.539939 & 15.351554 \\
\hline OW & -8.351653 & 0.006769 & 6.080028 \\
\hline HW & -8.411650 & 0.924832 & 5.688160 \\
\hline HW & -7.400013 & -0.195342 & 6.311400 \\
\hline $\mathrm{OW}$ & 9.999563 & 8.980100 & -6.624192 \\
\hline HW & 9.018594 & 9.028292 & -6.436103 \\
\hline HW & 10.143564 & 8.705539 & -7.574918 \\
\hline $\mathrm{OW}$ & -8.531004 & -1.767589 & 21.681886 \\
\hline HW & -8.554574 & -2.759027 & 21.553457 \\
\hline HW & -8.403924 & -1.560063 & 22.651826 \\
\hline OW & 10.128683 & 8.219234 & 20.329999 \\
\hline HW & -10.005839 & 7.955493 & 21.096587 \\
\hline HW & 9.632298 & 9.055732 & 20.562102 \\
\hline OW & 8.277652 & 5.577361 & 16.735736 \\
\hline HW & 7.313769 & 5.344363 & 16.864738 \\
\hline HW & 8.350158 & 6.380663 & 16.144593 \\
\hline $\mathrm{OW}$ & -5.582091 & -17.454026 & 8.062015 \\
\hline HW & -6.076485 & -16.585453 & 8.028027 \\
\hline HW & -5.793009 & 17.890179 & 7.244434 \\
\hline OW & -8.553926 & -15.566652 & -14.313868 \\
\hline HW & -8.708254 & -16.455510 & -13.882457 \\
\hline
\end{tabular}




\begin{tabular}{|c|c|c|c|}
\hline HW & -9.230051 & -15.425222 & -15.036953 \\
\hline $\mathrm{OW}$ & 1.943629 & -14.897791 & -2.109199 \\
\hline HW & 2.549483 & -14.959279 & -2.902396 \\
\hline HW & 2.079548 & -15.696652 & -1.523241 \\
\hline OW & -2.039348 & 13.577802 & 18.216903 \\
\hline HW & -1.047081 & 13.461322 & 18.259795 \\
\hline HW & -2.253077 & 14.500683 & 17.896573 \\
\hline OW & -0.282741 & -14.680946 & -14.429279 \\
\hline HW & -1.113423 & -14.505095 & -14.957525 \\
\hline HW & 0.429898 & -15.032930 & -15.036118 \\
\hline OW & 10.183419 & -7.780604 & -0.559914 \\
\hline HW & 9.668818 & -8.638016 & 4463 \\
\hline HW & -9.890149 & -7.784686 & -1.322874 \\
\hline OW & -4.422398 & 8.172009 & 4.771810 \\
\hline HW & -4.851955 & 7.417515 & 5.2 \\
\hline HW & -4.723493 & 8.158123 & 3.8 \\
\hline OW & -9.806078 & -16.093906 & 6.118753 \\
\hline HW & -9.938370 & -16.638489 & 6.946961 \\
\hline HW & -10.355192 & -15.260176 & 6.176804 \\
\hline $\mathrm{OW}$ & -9.607094 & -15.360073 & -11.395234 \\
\hline HW & -10.077392 & -15.909024 & -12.086228 \\
\hline HW & -9.16 & -14.5 & -11.834949 \\
\hline $\mathrm{OW}$ & 1.229 & -13.3 & 70428 \\
\hline HW & 1.874444 & -14.073803 & -11.783681 \\
\hline HW & 0.787292 & -13.643319 & -12.923598 \\
\hline OW & 9.257344 & 2900 & 2.426366 \\
\hline HW & 9.98 & -17.4 & 129 \\
\hline HW & 8.373671 & & \\
\hline OW & 8.538685 & -12.560111 & -11.944294 \\
\hline HW & 7.709095 & -12.648799 & -12.495578 \\
\hline HW & $8.63^{\circ}$ & -13.3 & -11.358488 \\
\hline $\mathrm{OW}$ & $7.67^{\prime}$ & 4.4 & -19.0 \\
\hline HW & 7.210036 & & -18.385292 \\
\hline HW & 7.152467 & 4.50 & 6189 \\
\hline OW & -0.060464 & 7657 & -15.714346 \\
\hline HW & 0.090500 & 16.271237 & -14.746996 \\
\hline HW & 0.156271 & 16.875323 & -16.262708 \\
\hline OW & 8.149149 & -11.598886 & 6728 \\
\hline HW & 9.005198 & -11.104615 & 7980 \\
\hline HW & 7.845067 & -11.458259 & 14.644519 \\
\hline OW & -5.694166 & 12.381364 & -14.792011 \\
\hline HW & -4.850203 & 11.979442 & -15.147237 \\
\hline HW & -5.517745 & 13.320457 & -14.497087 \\
\hline OW & -1.638714 & -3.247884 & -8.922758 \\
\hline HW & -1.434908 & -3.675285 & -9.803548 \\
\hline HW & -1.982991 & -3.939858 & -8.288216 \\
\hline OW & -7.541968 & -16.815728 & -0.213076 \\
\hline HW & -8.266574 & -16.635664 & -0.878300 \\
\hline HW & -7.485215 & -16.054010 & 0.432342 \\
\hline OW & 5.132362 & 10.416421 & 9.879626 \\
\hline HW & 5.902618 & 9.799016 & 9.719888 \\
\hline HW & 5.216655 & 11.215469 & 9.284297 \\
\hline
\end{tabular}




\begin{tabular}{|c|c|c|c|}
\hline OW & 0.007121 & 10.541511 & -18.534025 \\
\hline HW & 0.237103 & 11.070867 & -19.350658 \\
\hline HW & 0.088525 & 9.565437 & -18.735653 \\
\hline OW & -5.008760 & -8.974379 & -21.763817 \\
\hline HW & -4.970200 & -9.448351 & -22.643512 \\
\hline HW & -5.944489 & -8.666409 & -21.591868 \\
\hline OW & 9.426418 & 9.795884 & -21.606547 \\
\hline HW & -10.350005 & 9.545464 & -21.823235 \\
\hline HW & 8.875385 & 9.772409 & -22.440700 \\
\hline OW & 3.682200 & 6.479007 & 3.610227 \\
\hline HW & 3.126160 & 5.976797 & 4.272498 \\
\hline HW & 3.095778 & 7.088623 & 3.076863 \\
\hline OW & -0.773336 & 1.764230 & 17.393542 \\
\hline HW & -0.127270 & 1.642676 & 16.640001 \\
\hline HW & -0.317078 & 2.229634 & 18.151980 \\
\hline OW & 1.523283 & 15.665945 & 17.392314 \\
\hline HW & 2.312672 & 15.063042 & 17.276674 \\
\hline HW & 1.832084 & 16.616436 & 17.427103 \\
\hline OW & 0.779664 & 12.080363 & -9.235991 \\
\hline HW & 0.364762 & 12.517152 & -8.437823 \\
\hline HW & 0.392105 & 11.166204 & -9.354785 \\
\hline OW & -3.044532 & 10.665213 & 1.137426 \\
\hline HW & -2.106223 & 10.954346 & 0.947745 \\
\hline HW & -3.639992 & 11.467832 & 1.172489 \\
\hline OW & 2.341727 & -1.907645 & -1.284827 \\
\hline HW & 2.300407 & -2.297136 & -2.204931 \\
\hline HW & 1.414867 & -1.809613 & -0.922446 \\
\hline OW & -6.921003 & -13.143503 & -6.322680 \\
\hline HW & -7.703707 & -13.609828 & -5.910472 \\
\hline HW & -6.886632 & -13.350669 & -7.300382 \\
\hline OW & -7.416742 & -7.483356 & -12.717652 \\
\hline HW & -7.213148 & -7.697364 & -11.762272 \\
\hline HW & -6.705233 & -6.883621 & -13.083814 \\
\hline OW & -6.697209 & 17.355167 & -3.321903 \\
\hline HW & -7.613889 & 17.568301 & -3.659946 \\
\hline HW & -6.767002 & 16.755348 & -2.524817 \\
\hline OW & 5.847945 & 14.865201 & 0.854394 \\
\hline HW & 5.254604 & 15.669647 & 0.825882 \\
\hline HW & 5.645066 & 14.330293 & 1.674585 \\
\hline OW & -10.014101 & -10.770343 & 1.972994 \\
\hline HW & -9.995882 & -11.514369 & 2.640896 \\
\hline HW & -10.216861 & -11.140967 & 1.066613 \\
\hline OW & -9.899162 & -12.010494 & -9.631400 \\
\hline HW & 9.978750 & -12.215964 & -10.130068 \\
\hline HW & -9.994297 & -11.131250 & -9.164623 \\
\hline OW & -2.455612 & -8.084229 & -11.872445 \\
\hline HW & -1.531008 & -7.703378 & -11.864771 \\
\hline HW & -3.050465 & -7.526940 & -11.293153 \\
\hline OW & -7.235774 & -13.949084 & 21.806460 \\
\hline HW & -6.306995 & -13.641450 & 21.599741 \\
\hline HW & -7.341813 & -14.047521 & 22.795937 \\
\hline OW & -6.897406 & -6.619340 & 21.712394 \\
\hline
\end{tabular}




\begin{tabular}{|c|c|c|c|}
\hline HW & -6.370472 & -7.320522 & 2097 \\
\hline HW & -7.174149 & -6.965887 & 22.608674 \\
\hline OW & 9.063908 & -4.962027 & 2.377278 \\
\hline HW & 8.569001 & -4.932580 & 1.508831 \\
\hline HW & 8.412039 & -4.915107 & 3.134157 \\
\hline OW & 0.962015 & 4.354165 & -16.640400 \\
\hline HW & 1.369902 & 4.009199 & -15.795044 \\
\hline HW & 1.147463 & 5.333200 & -16.724650 \\
\hline OW & -1.018273 & -6.581777 & 21.146035 \\
\hline HW & -0.470638 & -5.751105 & 21.246431 \\
\hline HW & -0.657764 & -7.129452 & 20.390995 \\
\hline OW & -8.464117 & -12.885482 & 7.634371 \\
\hline HW & -8.673659 & -12.130044 & 7.013560 \\
\hline HW & -9.314722 & -13.323087 & 7.925873 \\
\hline OW & -9.723138 & 8.251439 & -9.064561 \\
\hline HW & -10.066538 & 7.550256 & -9.689396 \\
\hline HW & -8.815517 & 8.547501 & -9.362172 \\
\hline OW & -4.787276 & -5.919044 & 15.424257 \\
\hline HW & -5.428828 & -5.648984 & 14.706288 \\
\hline HW & -4.221283 & -6.676137 & 15.097972 \\
\hline OW & 7.072712 & 9.781195 & 6.687065 \\
\hline HW & 6.258245 & 9.237629 & 6.484137 \\
\hline HW & 7.809236 & 9.524422 & 6.061285 \\
\hline OW & 3.453471 & -3.809265 & 6.544464 \\
\hline HW & 3.702962 & -4.291516 & 5.704709 \\
\hline HW & 2.458212 & -3.801867 & 6.641440 \\
\hline OW & -8.167312 & -4.176194 & 11.702234 \\
\hline HW & -8.574761 & -3.266194 & 11.778953 \\
\hline HW & -8.834955 & -4.808241 & 11.308827 \\
\hline OW & 1.682166 & 2.617647 & -14.468510 \\
\hline HW & 1.389704 & 1.675760 & -14.303236 \\
\hline HW & 2.646883 & 2.625797 & -14.731671 \\
\hline OW & -0.162953 & -15.860195 & -19.300602 \\
\hline HW & 0.156247 & -15.524813 & -20.186959 \\
\hline HW & 0.620844 & -16.153853 & -18.753403 \\
\hline OW & -0.714594 & -16.017517 & 17.577030 \\
\hline HW & -0.637253 & -15.718591 & 16.625893 \\
\hline HW & 0.180636 & -15.958726 & 18.018739 \\
\hline OW & -1.358768 & 13.898998 & -20.745053 \\
\hline HW & -0.891084 & 13.125437 & -21.172693 \\
\hline HW & -1.670918 & 14.532529 & -21.453008 \\
\hline OW & -7.948753 & -13.555360 & -12.701705 \\
\hline HW & -8.369835 & -12.666191 & -12.880781 \\
\hline HW & -8.153383 & -14.179061 & -13.456107 \\
\hline OW & -2.445329 & -16.858754 & 13.313631 \\
\hline HW & -2.950671 & -17.030692 & 14.159248 \\
\hline HW & -1.975456 & -17.694773 & 13.030276 \\
\hline OW & -6.497710 & -4.128971 & -6.859950 \\
\hline HW & -7.351495 & -4.622683 & -6.694721 \\
\hline HW & -6.477915 & -3.300687 & -6.299992 \\
\hline OW & 5.810613 & -17.725453 & -18.933807 \\
\hline HW & 6.661408 & 17.659824 & -19.110997 \\
\hline
\end{tabular}




\begin{tabular}{|c|c|c|c|}
\hline HW & 5.419848 & -17.413550 & -19.799844 \\
\hline OW & 7.773665 & -10.733504 & 5.722286 \\
\hline HW & 6.863581 & -10.351480 & 5.561644 \\
\hline HW & 7.728679 & -11.400398 & 6.466079 \\
\hline OW & -1.821533 & -3.108085 & 10.006332 \\
\hline HW & -2.296501 & -2.851153 & 10.847992 \\
\hline HW & -1.346965 & -3.977544 & 10.143540 \\
\hline OW & 4.774036 & 10.440491 & 12.818906 \\
\hline HW & 4.208792 & 11.202784 & 12.503615 \\
\hline HW & 4.368073 & 9.577651 & 12.517740 \\
\hline OW & -8.573356 & -8.124423 & 4.609571 \\
\hline HW & -8.001403 & -8.717977 & 4.043387 \\
\hline HW & -8.997733 & -8.664837 & 5.336109 \\
\hline OW & 4.281536 & 3.602517 & 5.905671 \\
\hline HW & 4.168792 & 2.709031 & 5.470964 \\
\hline HW & 5.089818 & 3.587203 & 6.494269 \\
\hline OW & -10.117396 & 11.383455 & -10.226703 \\
\hline HW & 9.628332 & 11.566785 & -10.357795 \\
\hline HW & -9.973738 & 10.398663 & -10.128994 \\
\hline OW & -9.182229 & 12.882257 & -7.967349 \\
\hline HW & -9.670581 & 12.324707 & -8.638654 \\
\hline HW & -9.519676 & 12.671116 & -7.049990 \\
\hline OW & -9.301284 & -9.528050 & -7.636130 \\
\hline HW & -9.618946 & -9.223893 & -6.738033 \\
\hline HW & -9.144944 & -8.732436 & -8.221414 \\
\hline OW & 3.431348 & -17.909791 & -10.138314 \\
\hline HW & 3.444131 & -17.640835 & -9.175246 \\
\hline HW & 2.841043 & 17.171283 & -10.253458 \\
\hline OW & -6.349096 & -7.101187 & 2.510397 \\
\hline HW & -5.524955 & -7.121793 & 3.076407 \\
\hline HW & -6.706029 & -8.029625 & 2.407430 \\
\hline OW & -8.987261 & -6.409037 & -15.623033 \\
\hline HW & -8.494710 & -7.277104 & -15.685105 \\
\hline HW & -9.335363 & -6.157997 & -16.526251 \\
\hline OW & -7.651415 & -12.200621 & 11.426505 \\
\hline HW & -6.676214 & -12.007869 & 11.317746 \\
\hline HW & -7.903345 & -12.979404 & 10.852023 \\
\hline OW & 4.891996 & -17.589344 & -12.636799 \\
\hline HW & 4.566090 & -17.586575 & -11.691401 \\
\hline HW & 5.605121 & -16.896795 & -12.745557 \\
\hline OW & 4.417490 & -12.147656 & -20.928185 \\
\hline HW & 5.298680 & -12.533610 & -21.201212 \\
\hline HW & 3.696590 & -12.513643 & -21.516707 \\
\hline OW & -2.088644 & -14.992795 & 22.094433 \\
\hline HW & -1.303177 & -15.591586 & 21.937939 \\
\hline HW & -2.387155 & -15.076204 & 23.045188 \\
\hline OW & -1.135550 & 0.509092 & 6.928663 \\
\hline HW & -0.544708 & 1.248108 & 7.252331 \\
\hline HW & -0.588439 & -0.314957 & 6.781647 \\
\hline OW & -5.535193 & -8.016634 & 7.952300 \\
\hline HW & -5.389882 & -7.767085 & 8.909698 \\
\hline HW & -4.837918 & -8.675537 & 7.670070 \\
\hline
\end{tabular}




\begin{tabular}{|c|c|c|c|}
\hline OW & 10.011563 & -13.866174 & -7.714669 \\
\hline HW & 10.338001 & -13.110824 & -8.282899 \\
\hline HW & -10.056889 & -14.032170 & -6.974445 \\
\hline OW & -1.695551 & 6.589462 & 12.985664 \\
\hline HW & -1.502034 & 5.962541 & 12.230998 \\
\hline HW & -2.169753 & 6.096580 & 13.715185 \\
\hline OW & 4.258420 & 8.056632 & 11.343046 \\
\hline HW & 3.674947 & 7.423015 & 10.835022 \\
\hline HW & 4.605211 & 8.760437 & 10.723051 \\
\hline OW & -1.663919 & -5.921770 & -4.157785 \\
\hline HW & -2.526446 & -5.416023 & -4.174179 \\
\hline HW & -1.172970 & -5.716090 & -3.311224 \\
\hline $\mathrm{OW}$ & -6.542870 & -2.550100 & -9.347468 \\
\hline HW & -5.590868 & -2.443134 & -9.634262 \\
\hline HW & -6.606260 & -3.281505 & -8.668477 \\
\hline OW & -6.367560 & -8.734410 & 15.598147 \\
\hline HW & -7.225550 & -8.681304 & 15.087233 \\
\hline HW & -5.614036 & -8.426880 & 15.017090 \\
\hline OW & -9.865614 & -6.734619 & -18.249875 \\
\hline HW & -9.192651 & -7.385080 & -18.602042 \\
\hline HW & 10.332267 & -6.350188 & -19.011193 \\
\hline OW & -8.946950 & 0.632827 & -19.909996 \\
\hline HW & -9.467606 & 1.148466 & -19.229530 \\
\hline HW & -9.438134 & 0.639910 & -20.781022 \\
\hline OW & -5.275633 & 8.182294 & -3.148328 \\
\hline HW & -6.088715 & 8.760847 & -3.212936 \\
\hline HW & -4.884882 & 8.257775 & -2.230931 \\
\hline OW & 10.019627 & 11.634355 & 0.222985 \\
\hline HW & 9.753669 & 10.683995 & 0.061484 \\
\hline HW & 9.902370 & 11.853280 & 1.191656 \\
\hline $\mathrm{OW}$ & -0.530343 & 9.201994 & -6.634170 \\
\hline HW & -0.600860 & 8.681165 & -5.783426 \\
\hline HW & 0.379900 & 9.074967 & -7.028278 \\
\hline $\mathrm{OW}$ & -2.130249 & -0.272182 & -2.020833 \\
\hline HW & -2.244211 & 0.719410 & -1.959525 \\
\hline $\mathrm{HW}$ & -1.379147 & -0.483324 & -2.646345 \\
\hline $\mathrm{OW}$ & -7.286410 & -12.770412 & 14.153794 \\
\hline HW & -6.308532 & -12.959396 & 14.064133 \\
\hline $\mathrm{HW}$ & -7.687096 & -12.649146 & 13.245639 \\
\hline OW & 1.895748 & 9.489922 & 6.837865 \\
\hline HW & 2.213020 & 10.052071 & 7.601623 \\
\hline $\mathrm{HW}$ & 1.957319 & 8.523468 & 7.087215 \\
\hline OW & 3.231464 & 11.273912 & 5.097047 \\
\hline $\mathrm{HW}$ & 2.819918 & 10.479127 & 5.543081 \\
\hline $\mathrm{HW}$ & 4.020708 & 11.582545 & 5.627933 \\
\hline OW & -5.766452 & 2.279149 & 2.438331 \\
\hline HW & -5.306222 & 2.276180 & 3.326126 \\
\hline $\mathrm{HW}$ & -5.256719 & 2.856597 & 1.800580 \\
\hline OW & -4.773929 & 1.091457 & 15.208014 \\
\hline HW & -5.297987 & 1.577452 & 15.907421 \\
\hline HW & -4.274127 & 0.335685 & 15.631106 \\
\hline OW & 3.088759 & -2.567213 & 10.439145 \\
\hline
\end{tabular}




\begin{tabular}{|c|c|c|c|}
\hline HW & 2.462788 & -3.069484 & 11.035705 \\
\hline HW & 2.587140 & -2.219035 & 9.647217 \\
\hline OW & -8.383439 & -5.500610 & 2.728417 \\
\hline HW & -8.078623 & -4.598051 & 3.032513 \\
\hline HW & -7.592691 & -6.105660 & 2.635511 \\
\hline $\mathrm{OW}$ & -7.355507 & 9.140727 & -10.051898 \\
\hline HW & -7.118479 & 9.877200 & -9.418317 \\
\hline HW & -7.165975 & 8.257729 & -9.622489 \\
\hline OW & -6.404292 & 14.585440 & 7.048125 \\
\hline HW & -6.319617 & 15.568908 & 6.888059 \\
\hline HW & -5.877055 & 14.088902 & 6.358581 \\
\hline OW & -7.102051 & 3.078780 & 22.316227 \\
\hline HW & -6.204912 & 3.021894 & 22.754296 \\
\hline HW & -7.805722 & 2.770525 & 22.956404 \\
\hline OW & 7.526314 & 2.105961 & 15.562415 \\
\hline HW & 8.250315 & 2.002936 & 14.880354 \\
\hline HW & 7.808395 & 2.775686 & 16.249365 \\
\hline OW & 3.692858 & -7.951566 & -16.216924 \\
\hline HW & 3.541350 & -7.242689 & -15.528055 \\
\hline HW & 3.268823 & -7.674504 & -17.079149 \\
\hline OW & 9.657581 & 3.269371 & 20.164778 \\
\hline HW & 9.403479 & 3.192859 & 21.128924 \\
\hline HW & 9.766682 & 4.233891 & 19.924366 \\
\hline OW & 9.812284 & -0.373213 & 7.022655 \\
\hline HW & 9.444201 & -1.291538 & 6.877075 \\
\hline HW & -9.931109 & -0.367621 & 6.807696 \\
\hline $\mathrm{OW}$ & -8.828624 & 9.840070 & 18.473997 \\
\hline HW & -9.302101 & 10.718761 & 18.535013 \\
\hline HW & -9.151354 & 9.236229 & 19.202846 \\
\hline $\mathrm{OW}$ & -2.552389 & -8.113675 & -20.776209 \\
\hline HW & -3.353051 & -8.563634 & -21.171783 \\
\hline HW & -2.760577 & -7.823663 & -19.842105 \\
\hline OW & -1.834580 & 10.268385 & 19.318367 \\
\hline HW & -1.691018 & 10.775089 & 20.168450 \\
\hline $\mathrm{HW}$ & -2.634256 & 9.675714 & 19.414591 \\
\hline OW & -9.656595 & 11.668447 & -5.596714 \\
\hline HW & -10.137054 & 10.977334 & -6.136642 \\
\hline HW & -10.320176 & 12.304959 & -5.203633 \\
\hline $\mathrm{OW}$ & -7.350139 & 11.038981 & 4.281428 \\
\hline HW & -6.666319 & 11.636963 & 4.699531 \\
\hline HW & -7.297442 & 11.117386 & 3.285901 \\
\hline OW & 10.144818 & 3.577651 & 8.764044 \\
\hline HW & 9.737698 & 2.664285 & 8.767984 \\
\hline HW & 9.809381 & 4.095490 & 9.551017 \\
\hline OW & 10.290219 & 2.255689 & -6.313821 \\
\hline $\mathrm{HW}$ & -9.852838 & 2.942563 & -6.755795 \\
\hline HW & -9.921842 & 1.823084 & -5.568938 \\
\hline OW & 7.561723 & -6.086671 & -3.512391 \\
\hline $\mathrm{HW}$ & 8.520459 & -6.230988 & -3.757335 \\
\hline HW & 7.128525 & -5.497410 & -4.194381 \\
\hline $\mathrm{OW}$ & 3.348277 & -6.784895 & 9.783011 \\
\hline HW & 2.461442 & -6.363605 & 9.593175 \\
\hline
\end{tabular}




\begin{tabular}{|c|c|c|c|}
\hline HW & 3.914829 & -6.147899 & 10.305750 \\
\hline OW & -1.286033 & -2.027736 & -14.015519 \\
\hline HW & -1.648738 & -1.200350 & -13.586708 \\
\hline HW & -2.014605 & -2.707869 & -14.096760 \\
\hline OW & 4.276078 & -12.679781 & -18.363080 \\
\hline HW & 4.222330 & -12.612309 & -19.359352 \\
\hline HW & 5.039097 & -12.124583 & -18.032083 \\
\hline OW & -2.457604 & -4.215395 & 14.327595 \\
\hline HW & -1.684307 & -4.381492 & 14.939498 \\
\hline HW & -3.276227 & -4.654016 & 14.698361 \\
\hline OW & -4.971077 & -11.128024 & 11.476385 \\
\hline HW & -4.834008 & -10.977035 & 12.455372 \\
\hline HW & -4.324888 & -11.818826 & 11.151987 \\
\hline OW & 2.283223 & 6.808993 & 7.318063 \\
\hline HW & 1.901128 & 6.169383 & 6.651052 \\
\hline HW & 2.754885 & 6.298539 & 8.037070 \\
\hline OW & -9.629813 & 7.633738 & 11.019267 \\
\hline HW & -9.782592 & 8.589290 & 10.767115 \\
\hline HW & -10.289679 & 7.364531 & 11.720768 \\
\hline OW & 0.817606 & -2.801614 & -15.464836 \\
\hline HW & 0.890226 & -2.511927 & -16.419198 \\
\hline HW & 0.017932 & -2.371503 & -15.045877 \\
\hline OW & 8.305507 & -1.662594 & -8.680533 \\
\hline HW & 7.362382 & -1.633896 & -9.011731 \\
\hline HW & 8.314521 & -1.534954 & -7.688754 \\
\hline OW & 7.318033 & 14.794170 & -21.607924 \\
\hline $\mathrm{HW}$ & 7.725082 & 13.889364 & -21.482877 \\
\hline HW & 7.089664 & 14.926512 & -22.572462 \\
\hline OW & -9.034999 & -7.895542 & 19.918914 \\
\hline HW & -8.046476 & -7.804555 & 20.039509 \\
\hline HW & -9.492814 & -7.093590 & 20.302682 \\
\hline $\mathrm{OW}$ & -0.475677 & -10.267032 & -15.678523 \\
\hline HW & -0.229574 & -10.464054 & -14.729515 \\
\hline HW & -1.241866 & -10.848644 & -15.951800 \\
\hline $\mathrm{OW}$ & -5.985684 & -15.493376 & -14.932481 \\
\hline HW & -6.928611 & -15.776082 & -14.756508 \\
\hline HW & -5.817870 & -14.606345 & -14.502349 \\
\hline OW & -9.224310 & -12.675432 & 3.775075 \\
\hline HW & -8.461823 & -13.164485 & 3.351471 \\
\hline HW & -9.809428 & -13.325768 & 4.259533 \\
\hline $\mathrm{OW}$ & 8.893155 & 12.608285 & -2.159393 \\
\hline HW & 9.362933 & 12.146324 & -1.407129 \\
\hline HW & 8.103885 & 13.108081 & -1.802662 \\
\hline OW & 3.880612 & -0.139618 & 0.057989 \\
\hline HW & 4.361564 & -0.518641 & 0.848575 \\
\hline $\mathrm{HW}$ & 3.358030 & -0.863363 & -0.392679 \\
\hline OW & 0.248684 & -8.383117 & 1.570727 \\
\hline HW & -0.027112 & -7.480149 & 1.241204 \\
\hline $\mathrm{HW}$ & -0.286341 & -8.619687 & 2.381765 \\
\hline OW & -2.920942 & -17.728074 & 17.337607 \\
\hline HW & -3.258671 & 17.783137 & 18.203595 \\
\hline HW & -2.159304 & -17.104866 & 17.515146 \\
\hline
\end{tabular}




\begin{tabular}{|c|c|c|c|}
\hline OW & -1.912194 & -0.762419 & 1.073644 \\
\hline HW & -2.910488 & -0.727038 & 1.120099 \\
\hline HW & -1.556426 & -1.217042 & 1.890190 \\
\hline OW & 4.766863 & -1.060233 & 18.367526 \\
\hline HW & 5.758077 & -1.185137 & 18.411047 \\
\hline HW & 4.484595 & -0.391904 & 19.055757 \\
\hline OW & 5.795911 & -13.645793 & 10.422005 \\
\hline HW & 5.514161 & -12.688743 & 10.490362 \\
\hline HW & 6.352294 & -13.887060 & 11.217133 \\
\hline OW & 8.693030 & -12.416767 & -4.581045 \\
\hline HW & 8.404398 & -12.444002 & -3.623993 \\
\hline HW & 9.645499 & -12.116685 & -4.633526 \\
\hline OW & -3.915797 & -16.576826 & -13.215876 \\
\hline HW & -4.736297 & -16.394100 & -13.757532 \\
\hline HW & -3.431739 & -17.363464 & -13.599137 \\
\hline OW & 3.279548 & -14.783115 & -19.886071 \\
\hline HW & 3.654682 & -14.175510 & -19.186006 \\
\hline HW & 2.501672 & -14.339078 & -20.330752 \\
\hline OW & 8.957122 & -4.230044 & 7.424759 \\
\hline HW & 9.287082 & -5.041980 & 6.943214 \\
\hline HW & 8.542440 & -3.599139 & 6.769016 \\
\hline $\mathrm{OW}$ & 3.882547 & 17.620037 & -3.839378 \\
\hline HW & 4.214057 & -17.371069 & -3.523198 \\
\hline HW & 2.985555 & 17.731727 & -4.267081 \\
\hline OW & 4.524781 & 9.771386 & -16.649620 \\
\hline HW & 4.555064 & 9.646263 & -15.657941 \\
\hline $\mathrm{HW}$ & 3.714381 & 10.303960 & -16.893786 \\
\hline OW & -7.354107 & 4.677620 & 5.486195 \\
\hline HW & -8.025277 & 4.875309 & 6.200653 \\
\hline HW & -7.411143 & 5.376084 & 4.772826 \\
\hline OW & -2.672565 & 17.120019 & -14.439122 \\
\hline $\mathrm{HW}$ & -2.554829 & 17.522642 & -15.346885 \\
\hline HW & -1.776956 & 16.893642 & -14.056187 \\
\hline OW & 1.471394 & -15.421982 & 19.247308 \\
\hline HW & 1.294399 & -14.439845 & 19.183437 \\
\hline HW & 1.147698 & -15.762279 & 20.130155 \\
\hline OW & 2.118875 & -1.645358 & 3.989050 \\
\hline HW & 2.557576 & -2.308006 & 3.382058 \\
\hline HW & 2.792738 & -1.289218 & 4.636409 \\
\hline OW & 4.650423 & -0.994698 & 2.467176 \\
\hline HW & 5.169239 & -1.844392 & 2.561252 \\
\hline HW & 5.037881 & -0.298888 & 3.071928 \\
\hline OW & -6.156529 & -6.367594 & -2.882054 \\
\hline HW & -6.239262 & -6.219239 & -3.867521 \\
\hline HW & -6.211298 & -7.346784 & -2.686638 \\
\hline $\mathrm{OW}$ & -3.472757 & -9.468298 & 6.048330 \\
\hline HW & -2.762986 & -9.902606 & 6.602948 \\
\hline HW & -3.558769 & -9.952984 & 5.177881 \\
\hline $\mathrm{OW}$ & -1.858964 & 4.580453 & 6.853081 \\
\hline HW & -1.615074 & 5.327763 & 6.234981 \\
\hline HW & -2.839407 & 4.396516 & 6.783082 \\
\hline OW & -8.352033 & -12.963029 & -17.373651 \\
\hline
\end{tabular}




\begin{tabular}{|c|c|c|c|}
\hline HW & -9.040612 & -12.997219 & -16.649295 \\
\hline HW & -8.121153 & -12.009929 & -17.569339 \\
\hline OW & 6.150727 & -10.026925 & 22.448502 \\
\hline HW & 5.670029 & -10.336481 & 23.268932 \\
\hline HW & 6.904398 & -9.424433 & 22.711145 \\
\hline $\mathrm{OW}$ & -7.123495 & 5.991259 & -17.319635 \\
\hline HW & -6.526620 & 5.208149 & -17.494215 \\
\hline HW & -6.592591 & 6.836151 & -17.385207 \\
\hline OW & 9.156701 & -1.964415 & -17.731927 \\
\hline HW & 8.555703 & -1.752073 & -16.961400 \\
\hline HW & 9.053140 & -2.927648 & -17.979834 \\
\hline OW & 6.537457 & -6.774412 & -21.101853 \\
\hline HW & 6.208439 & -6.858460 & -22.042430 \\
\hline HW & 6.064646 & -7.438552 & -20.522744 \\
\hline OW & -3.189686 & 7.459746 & 17.844892 \\
\hline HW & -3.637668 & 7.887957 & 18.629716 \\
\hline HW & -3.326945 & 6.469903 & 17.881917 \\
\hline OW & 3.868677 & 6.290093 & 15.005993 \\
\hline HW & 3.655926 & 7.200989 & 15.359556 \\
\hline HW & 4.793402 & 6.287898 & 14.625362 \\
\hline OW & 5.168651 & 10.905858 & 0.101101 \\
\hline HW & 4.784901 & 11.720595 & -0.333571 \\
\hline HW & 5.113978 & 10.133426 & -0.531640 \\
\hline OW & -0.156286 & -3.643650 & 2.235716 \\
\hline HW & 0.261090 & -4.334630 & 2.825922 \\
\hline HW & 0.558595 & -3.065873 & 1.841863 \\
\hline $\mathrm{OW}$ & -3.177384 & 12.008102 & -16.098079 \\
\hline HW & -2.480485 & 11.899148 & -15.389235 \\
\hline HW & -3.125814 & 11.240543 & -16.736979 \\
\hline $\mathrm{OW}$ & -10.200714 & -16.725631 & -16.822122 \\
\hline HW & 10.251644 & -16.187169 & -17.621138 \\
\hline HW & -9.281867 & -17.094797 & -16.961532 \\
\hline OW & -6.159225 & -14.838517 & 8.522960 \\
\hline HW & -5.225407 & -14.728814 & 8.863472 \\
\hline HW & -6.498520 & -13.958350 & 8.191017 \\
\hline OW & 3.373764 & 16.637842 & 7.970965 \\
\hline HW & 3.035157 & 17.528830 & 8.273433 \\
\hline HW & 3.754427 & 16.721058 & 7.050003 \\
\hline $\mathrm{OW}$ & 5.533269 & 12.643681 & -8.564536 \\
\hline HW & 4.759173 & 12.019667 & -8.671227 \\
\hline HW & 5.972119 & 12.482293 & -7.680588 \\
\hline OW & 2.686690 & -10.919014 & -0.883658 \\
\hline HW & 3.311187 & -11.696222 & -0.806513 \\
\hline HW & 1.782887 & -11.244008 & -1.162081 \\
\hline OW & 6.504172 & -2.540475 & 13.263114 \\
\hline HW & 6.738262 & -2.090880 & 14.125127 \\
\hline HW & 6.164646 & -3.462419 & 13.449501 \\
\hline OW & -7.260563 & 16.037625 & 11.311241 \\
\hline $\mathrm{HW}$ & -8.207487 & 16.153240 & 11.011296 \\
\hline HW & -6.983391 & 15.085734 & 11.180548 \\
\hline OW & -9.333526 & 14.950565 & -18.191377 \\
\hline $\mathrm{HW}$ & -8.583078 & 14.903981 & -17.532091 \\
\hline
\end{tabular}




\begin{tabular}{|c|c|c|c|}
\hline HW & -10.202326 & 15.029010 & 02468 \\
\hline OW & -5.080880 & -3.958304 & -21.465402 \\
\hline HW & -5.105502 & -2.994259 & -21.200804 \\
\hline HW & -5.424245 & -4.056450 & -22.399462 \\
\hline OW & 8.004194 & 12.748923 & -10.186521 \\
\hline HW & 7.335929 & 12.854550 & -9.450135 \\
\hline HW & 7.569327 & 12.954377 & -11.063264 \\
\hline OW & -9.566192 & 5.209851 & -16.372584 \\
\hline HW & -9.765740 & 5.244625 & -15.393313 \\
\hline HW & -8.793295 & 5.810625 & -16.576792 \\
\hline OW & 5.632849 & -3.631343 & 1.702229 \\
\hline HW & 6.153768 & -3.421243 & 0.874884 \\
\hline HW & 5.463898 & -4.615871 & 1.748708 \\
\hline OW & 9.252896 & -15.293430 & -19.284692 \\
\hline HW & 9.718129 & -14.430484 & -19.481876 \\
\hline HW & 9.480393 & -15.963733 & -19.991049 \\
\hline OW & 8.404582 & 8.697886 & 2.419252 \\
\hline HW & 8.341453 & 8.950770 & 1.453817 \\
\hline HW & 7.517854 & 8.359029 & 2.733721 \\
\hline OW & -5.496281 & -5.772248 & -13.463368 \\
\hline HW & -6.135924 & -5.112893 & -13.858473 \\
\hline HW & -4.696149 & -5.288793 & -13.108318 \\
\hline OW & 8.556603 & 5.829765 & 10.366931 \\
\hline HW & 8.926194 & 6.577657 & 10.918348 \\
\hline HW & 8.307859 & 5.066954 & 10.963794 \\
\hline OW & 6.663061 & -0.339554 & 21.647261 \\
\hline HW & 6.496920 & -0.112915 & 22.606965 \\
\hline HW & 7.139164 & -1.217052 & 21.589601 \\
\hline OW & 4.338247 & -11.981156 & 18.294614 \\
\hline HW & 4.003425 & -12.879542 & 18.578860 \\
\hline HW & 4.973399 & -11.629307 & 18.982207 \\
\hline OW & -2.189878 & 5.636114 & 9.553859 \\
\hline HW & -1.319220 & 5.754648 & 10.031251 \\
\hline HW & -2.058516 & 5.035438 & 8.765232 \\
\hline OW & -9.174936 & -16.725847 & 16.510187 \\
\hline HW & -8.511614 & -16.026707 & 16.777034 \\
\hline HW & -9.937824 & -16.294852 & 16.028269 \\
\hline OW & 8.582913 & 15.525220 & 1.201308 \\
\hline HW & 7.616588 & 15.431088 & 0.961818 \\
\hline HW & 8.675430 & 16.155756 & 1.971934 \\
\hline OW & 2.795940 & 4.983242 & -11.173317 \\
\hline HW & 3.711253 & 4.853712 & -10.791973 \\
\hline HW & 2.324706 & 5.707697 & -10.670223 \\
\hline OW & -10.207655 & 12.649917 & 4.389846 \\
\hline HW & -9.288963 & 12.837818 & 4.042430 \\
\hline HW & 9.841169 & 12.826363 & 3.669854 \\
\hline OW & -0.930633 & -1.729852 & 15.590284 \\
\hline HW & -0.442733 & -1.287631 & 14.837692 \\
\hline HW & -0.902207 & -2.722015 & 15.468606 \\
\hline OW & -4.397712 & -6.790037 & -7.526552 \\
\hline HW & -4.590770 & -7.743139 & -7.759632 \\
\hline HW & -4.584490 & -6.208804 & -8.318563 \\
\hline
\end{tabular}




\begin{tabular}{|c|c|c|c|}
\hline OW & -1.298216 & 5.762992 & -19.829114 \\
\hline HW & -1.570702 & 5.378114 & -20.710942 \\
\hline HW & -1.936237 & 5.459388 & -19.121475 \\
\hline OW & 2.027680 & 6.545227 & -4.395377 \\
\hline HW & 2.453419 & 7.448895 & -4.441542 \\
\hline HW & 1.192646 & 6.597764 & -3.847694 \\
\hline OW & 10.055028 & -12.118769 & 21.542516 \\
\hline HW & 9.967398 & -12.575333 & 22.427881 \\
\hline HW & 10.324449 & -11.166187 & 21.683938 \\
\hline OW & 10.227679 & 6.042693 & 18.513017 \\
\hline HW & 10.095177 & 6.952729 & 18.905799 \\
\hline HW & 9.453379 & 5.819226 & 17.920967 \\
\hline $\mathrm{OW}$ & -3.038353 & -17.629823 & -4.808065 \\
\hline HW & -3.451758 & 17.373413 & -4.562342 \\
\hline HW & -2.391467 & -17.765013 & -5.558573 \\
\hline OW & 1.128239 & 3.159797 & 0.387595 \\
\hline HW & 0.836215 & 3.975022 & -0.112535 \\
\hline HW & 1.878222 & 2.717730 & -0.104444 \\
\hline OW & 0.698445 & 7.805837 & -19.309455 \\
\hline HW & 0.484826 & 8.311914 & -20.145071 \\
\hline HW & 0.415766 & 6.852521 & -19.415668 \\
\hline OW & -0.477216 & -0.587440 & 9.751906 \\
\hline HW & -1.107088 & -1.336615 & 9.546975 \\
\hline HW & 0.355483 & -0.693991 & 9.208527 \\
\hline OW & 8.820836 & 0.631009 & 19.745588 \\
\hline HW & 8.159882 & 0.277765 & 20.407674 \\
\hline HW & 9.165619 & 1.515994 & 20.058521 \\
\hline OW & -7.056258 & -16.273018 & 12.914009 \\
\hline HW & -6.361879 & -16.846474 & 12.479285 \\
\hline HW & -6.615439 & -15.641719 & 13.552084 \\
\hline OW & -1.982902 & -15.257020 & -12.141700 \\
\hline HW & -1.247321 & -15.345986 & -12.813270 \\
\hline HW & -2.785182 & -15.763919 & -12.456979 \\
\hline $\mathrm{OW}$ & 3.145542 & 13.909242 & 8.255851 \\
\hline HW & 2.978590 & 14.882781 & 8.411895 \\
\hline HW & 2.647564 & 13.612051 & 7.441176 \\
\hline OW & 8.306822 & 16.745716 & -20.019582 \\
\hline HW & 8.091001 & 16.202534 & -20.830985 \\
\hline HW & 9.113977 & 17.307871 & -20.199813 \\
\hline OW & -1.810829 & -13.915879 & 7.833638 \\
\hline HW & -2.235061 & -14.816841 & 7.924722 \\
\hline HW & -2.522861 & -13.220454 & 7.736713 \\
\hline OW & -3.741118 & -2.603146 & -1.640873 \\
\hline HW & -3.180197 & -1.777496 & -1.701444 \\
\hline HW & -3.455102 & -3.143080 & -0.849250 \\
\hline OW & -5.223278 & -13.356516 & -21.250695 \\
\hline HW & -5.422722 & -14.264692 & -20.882676 \\
\hline HW & -4.855608 & -13.445367 & -22.176398 \\
\hline OW & 9.194421 & -5.287973 & 18.217426 \\
\hline HW & 9.612943 & -4.524856 & 17.724992 \\
\hline HW & 8.214887 & -5.316144 & 18.018127 \\
\hline $\mathrm{OW}$ & 7.402468 & -13.672686 & 19.278707 \\
\hline
\end{tabular}




\begin{tabular}{|c|c|c|c|}
\hline HW & 6.764920 & -14.260592 & 18.780812 \\
\hline HW & 6.921389 & -12.857766 & 19.601924 \\
\hline $\mathrm{OW}$ & -4.178299 & -2.237244 & 20.794507 \\
\hline HW & -4.094419 & -2.801998 & 21.615493 \\
\hline HW & -3.902645 & -1.299424 & 21.005476 \\
\hline OW & 2.142155 & 7.193717 & -14.446054 \\
\hline HW & 1.720288 & 6.338381 & -14.145340 \\
\hline HW & 2.044880 & 7.282882 & -15.437310 \\
\hline OW & -4.570367 & -16.062574 & 4.631818 \\
\hline HW & -4.599319 & -15.863148 & 3.652333 \\
\hline HW & -5.483538 & -15.946940 & 5.022648 \\
\hline OW & -10.215772 & 6.544866 & 2.647685 \\
\hline HW & 10.009309 & 7.373520 & 2.386176 \\
\hline HW & -9.362577 & 6.796407 & 3.104615 \\
\hline $\mathrm{OW}$ & -8.963769 & 15.802094 & 14.673114 \\
\hline HW & -8.879908 & 15.504089 & 15.623987 \\
\hline HW & -8.990099 & 15.001797 & 14.074088 \\
\hline OW & -9.858855 & 8.827349 & -3.573696 \\
\hline HW & -9.270229 & 8.550942 & -4.333379 \\
\hline HW & 10.305159 & 9.610970 & -3.850855 \\
\hline OW & -5.653130 & 8.314482 & -17.403101 \\
\hline HW & -5.028558 & 8.609199 & -16.679878 \\
\hline HW & -5.126328 & 7.942028 & -18.167141 \\
\hline OW & 5.284371 & 15.311545 & -19.747084 \\
\hline HW & 5.958644 & 15.337414 & -20.485112 \\
\hline HW & 5.567829 & 14.642609 & -19.059933 \\
\hline $\mathrm{OW}$ & -0.741080 & -4.516476 & 18.712851 \\
\hline HW & -1.643432 & -4.129245 & 18.902098 \\
\hline HW & -0.169751 & -4.445486 & 19.530496 \\
\hline OW & 0.401604 & -17.925029 & 5.164087 \\
\hline HW & -0.294735 & -17.262654 & 4.887732 \\
\hline HW & 1.265143 & -17.449909 & 5.333090 \\
\hline OW & 8.059863 & 16.233889 & 10.759760 \\
\hline $\mathrm{HW}$ & 7.397809 & 16.176648 & 10.012492 \\
\hline HW & 7.617687 & 16.623348 & 11.567722 \\
\hline $\mathrm{OW}$ & 9.106536 & -7.378208 & 12.839134 \\
\hline HW & 8.721862 & -8.175858 & 12.374611 \\
\hline HW & 8.365007 & -6.815594 & 13.204650 \\
\hline $\mathrm{OW}$ & 5.350575 & 11.972572 & 17.223261 \\
\hline HW & 6.218344 & 11.926690 & 16.728415 \\
\hline HW & 5.445888 & 11.513511 & 18.106538 \\
\hline $\mathrm{OW}$ & 3.402753 & -7.082428 & 14.223500 \\
\hline HW & 4.020562 & -7.689565 & 14.723197 \\
\hline $\mathrm{HW}$ & 2.466685 & -7.426835 & 14.295334 \\
\hline $\mathrm{OW}$ & -2.923932 & -11.060707 & 20.167011 \\
\hline HW & -2.064437 & -11.172017 & 20.665888 \\
\hline HW & -2.846035 & -11.492396 & 19.268358 \\
\hline $\mathrm{OW}$ & -6.108946 & 4.335056 & -15.108998 \\
\hline $\mathrm{HW}$ & -6.689564 & 3.572183 & -15.393441 \\
\hline HW & -6.682678 & 5.112077 & -14.850022 \\
\hline OW & -8.152270 & 15.120968 & -9.416303 \\
\hline HW & -8.965164 & 15.447120 & -9.898826 \\
\hline
\end{tabular}




\begin{tabular}{|c|c|c|c|}
\hline HW & -8.345327 & 14.231891 & -9.001249 \\
\hline OW & 4.371845 & 0.911034 & 8.776055 \\
\hline HW & 5.006282 & 0.252073 & 8.372000 \\
\hline HW & 4.485587 & 0.917180 & 9.769547 \\
\hline OW & -6.426282 & 9.644616 & 17.063778 \\
\hline HW & -7.172221 & 9.758593 & 17.719968 \\
\hline HW & -5.643027 & 10.196312 & 17.350384 \\
\hline OW & -9.253698 & -11.784484 & -4.368932 \\
\hline HW & -8.345280 & -11.768594 & -4.786694 \\
\hline HW & -9.272077 & -12.465556 & -3.636946 \\
\hline OW & 3.448234 & 16.667637 & -18.097405 \\
\hline HW & 4.162758 & 16.243599 & -18.653866 \\
\hline HW & 3.826284 & 16.922914 & -17.207510 \\
\hline OW & 5.591717 & 12.163620 & 6.274769 \\
\hline HW & 5.420139 & 12.578617 & 7.168268 \\
\hline HW & 6.215543 & 11.389330 & 6.381148 \\
\hline OW & -1.545186 & 3.999242 & -15.680193 \\
\hline HW & -0.690082 & 4.157248 & -16.173985 \\
\hline HW & -1.373577 & 3.396618 & -14.900838 \\
\hline OW & 9.195818 & 2.277269 & -10.966730 \\
\hline HW & 9.095146 & 1.755202 & -10.119788 \\
\hline HW & 8.334667 & 2.739875 & -11.177483 \\
\hline OW & 2.962970 & -11.536268 & -5.983993 \\
\hline HW & 2.889023 & -10.539746 & -6.022427 \\
\hline HW & 2.790733 & -11.847479 & -5.049390 \\
\hline OW & 7.143221 & 8.702616 & -6.143175 \\
\hline HW & 6.698838 & 9.598453 & -6.143792 \\
\hline HW & 6.455209 & 7.989348 & -6.009432 \\
\hline OW & -5.198913 & 6.708005 & 7.433649 \\
\hline HW & -5.634460 & 5.937667 & 7.899350 \\
\hline HW & -4.634236 & 7.215079 & 8.084814 \\
\hline OW & -4.515991 & -15.980089 & 21.075087 \\
\hline HW & -3.654023 & -15.701797 & 21.498840 \\
\hline HW & -5.042817 & -16.531270 & 21.722122 \\
\hline OW & 0.975422 & 0.177070 & 2.012833 \\
\hline HW & 1.969083 & 0.280770 & 1.969406 \\
\hline HW & 0.716051 & -0.175242 & 2.912057 \\
\hline OW & 4.773670 & -4.708700 & 10.537752 \\
\hline HW & 5.265562 & -4.930248 & 9.695755 \\
\hline HW & 4.385890 & -3.789800 & 10.465289 \\
\hline OW & -4.660350 & 6.781765 & 21.831032 \\
\hline HW & -4.435394 & 7.008228 & 22.778718 \\
\hline HW & -5.633530 & 6.562061 & 21.762828 \\
\hline OW & -5.497101 & 14.732523 & -18.972810 \\
\hline HW & -6.079712 & 14.781930 & -19.784058 \\
\hline HW & -4.802839 & 14.024792 & -19.103644 \\
\hline OW & 7.684586 & -13.192944 & -15.994196 \\
\hline HW & 7.699268 & -14.156272 & -16.262122 \\
\hline HW & 7.618381 & -12.620624 & -16.811550 \\
\hline OW & 5.601704 & -2.116526 & -9.402210 \\
\hline HW & 5.891349 & -2.992373 & -9.788212 \\
\hline HW & 5.042873 & -1.626065 & -10.070906 \\
\hline
\end{tabular}




\begin{tabular}{|c|c|c|c|}
\hline OW & 10.355334 & 2.147563 & 17.263731 \\
\hline HW & 9.777348 & 1.831205 & 18.015961 \\
\hline HW & -9.972840 & 3.037779 & 17.496086 \\
\hline OW & -9.203892 & 0.013219 & 19.837257 \\
\hline HW & -10.152243 & 0.330414 & 19.841241 \\
\hline HW & -9.040501 & -0.559180 & 20.640789 \\
\hline OW & 6.472041 & 11.735920 & -5.984153 \\
\hline HW & 7.434983 & 11.987280 & -5.886373 \\
\hline HW & 5.988070 & 11.932198 & -5.131365 \\
\hline OW & -8.780395 & 4.187220 & -12.087671 \\
\hline HW & -8.168244 & 4.932586 & -12.351680 \\
\hline HW & -9.680481 & 4.333941 & -12.497939 \\
\hline OW & 1.265543 & 9.852641 & 13.401353 \\
\hline HW & 0.819467 & 9.083348 & 13.858741 \\
\hline HW & 0.659760 & 10.648061 & 13.419658 \\
\hline OW & 5.171722 & 9.138654 & -21.947306 \\
\hline HW & 4.203810 & 9.045583 & -22.180725 \\
\hline HW & 5.729748 & 8.932632 & -22.751148 \\
\hline OW & -8.884745 & 2.708360 & -9.695306 \\
\hline HW & -9.600605 & 2.010426 & -9.674545 \\
\hline HW & -8.921445 & 3.197654 & -10.566653 \\
\hline OW & -8.243052 & -5.631187 & -0.455898 \\
\hline HW & -7.765478 & -4.848723 & -0.056307 \\
\hline HW & -7.610801 & -6.148533 & -1.032624 \\
\hline OW & 1.964261 & 7.867464 & 17.906373 \\
\hline HW & 2.842890 & 7.580884 & 18.288320 \\
\hline HW & 1.371979 & 8.195489 & 18.642310 \\
\hline OW & 2.712153 & 9.340794 & -5.137619 \\
\hline HW & 3.341265 & 9.459703 & -4.369453 \\
\hline HW & 1.955246 & 9.988674 & -5.051924 \\
\hline OW & -4.671405 & -7.397852 & 4.752477 \\
\hline HW & -5.286925 & -6.792949 & 5.257678 \\
\hline HW & -4.342193 & -8.120995 & 5.359670 \\
\hline OW & -3.316961 & -8.351758 & 1.083634 \\
\hline HW & -4.179877 & -7.846483 & 1.092179 \\
\hline HW & -3.371467 & -9.093201 & 0.414836 \\
\hline OW & -0.502625 & -5.607128 & -1.492816 \\
\hline HW & 0.436830 & -5.509402 & -1.164371 \\
\hline HW & -1.102820 & -5.829893 & -0.724609 \\
\hline OW & 5.738371 & 4.757283 & 16.591923 \\
\hline HW & 5.867016 & 5.065604 & 17.534467 \\
\hline HW & 5.088428 & 5.360522 & 16.129672 \\
\hline OW & -5.300969 & 15.172928 & -14.585368 \\
\hline HW & -5.519490 & 15.516346 & -13.671961 \\
\hline HW & -4.339462 & 15.356943 & -14.789436 \\
\hline OW & 3.065464 & -9.242311 & 17.647213 \\
\hline HW & 3.490877 & -10.133173 & 17.806551 \\
\hline HW & 2.477103 & -9.010566 & 18.421891 \\
\hline OW & -9.776038 & -6.463478 & -11.305397 \\
\hline HW & -9.702131 & -7.041833 & -12.117828 \\
\hline HW & -9.877051 & -5.508453 & -11.584185 \\
\hline OW & -6.245555 & 16.016490 & -11.937175 \\
\hline
\end{tabular}




\begin{tabular}{|c|c|c|c|}
\hline HW & -6.911659 & 16.685186 & -11.606794 \\
\hline HW & -6.427033 & 15.128500 & -11.514636 \\
\hline OW & 3.057102 & -13.593544 & 0.940350 \\
\hline HW & 2.544388 & -14.448163 & 1.022513 \\
\hline HW & 3.187271 & -13.194368 & 1.847937 \\
\hline OW & 4.951043 & 6.761850 & -6.273657 \\
\hline HW & 4.531720 & 6.279912 & -5.504304 \\
\hline HW & 4.415201 & 6.597589 & -7.101843 \\
\hline OW & 4.587219 & 7.108122 & -18.946423 \\
\hline HW & 4.082342 & 6.901347 & -19.784482 \\
\hline HW & 5.560149 & 7.201421 & -19.157852 \\
\hline OW & 1.764427 & -16.468803 & 1.228280 \\
\hline HW & 1.026402 & -16.926281 & 0.732262 \\
\hline HW & 2.136819 & -17.089283 & 1.918445 \\
\hline OW & 9.630864 & -13.235410 & 5.358417 \\
\hline HW & 9.469851 & -12.257730 & 5.223453 \\
\hline HW & 8.756879 & -13.721305 & 5.350902 \\
\hline OW & -8.878390 & -2.673886 & -20.590857 \\
\hline HW & -9.568770 & -2.123251 & -21.060087 \\
\hline HW & -8.789158 & -3.558074 & -21.049387 \\
\hline OW & -3.082663 & 8.000498 & 2.086528 \\
\hline HW & -3.479395 & 8.855134 & 1.751564 \\
\hline HW & -2.391802 & 8.203199 & 2.780519 \\
\hline OW & -7.639602 & 9.295762 & -21.365876 \\
\hline HW & -6.725872 & 9.669491 & -21.206425 \\
\hline HW & -7.818870 & 9.261682 & -22.349086 \\
\hline OW & -3.456529 & 0.812531 & -9.821426 \\
\hline HW & -3.514868 & 1.810187 & -9.785639 \\
\hline HW & -3.717386 & 0.431365 & -8.934484 \\
\hline OW & 0.416559 & 8.996679 & -12.966145 \\
\hline HW & 0.229005 & 9.774639 & -13.565813 \\
\hline HW & 0.804551 & 8.247726 & -13.503296 \\
\hline OW & -5.420295 & 1.189409 & -12.360644 \\
\hline HW & -6.418697 & 1.165423 & -12.309478 \\
\hline HW & -5.034242 & 0.810148 & -11.519737 \\
\hline OW & 6.315220 & 8.544503 & 17.116249 \\
\hline HW & 6.436455 & 8.734627 & 18.090495 \\
\hline HW & 5.385243 & 8.789380 & 16.842062 \\
\hline OW & -0.573108 & 0.686880 & -21.754124 \\
\hline HW & -0.485264 & 0.878741 & -22.731606 \\
\hline HW & -1.105283 & 1.412823 & -21.318468 \\
\hline OW & -3.763010 & -15.502696 & 11.544577 \\
\hline HW & -3.111990 & -16.036871 & 12.083864 \\
\hline HW & -4.371439 & -16.121666 & 11.047899 \\
\hline OW & 2.824320 & -10.270322 & 9.336305 \\
\hline HW & 3.419553 & -10.697644 & 10.016814 \\
\hline HW & 1.880516 & -10.278823 & 9.666702 \\
\hline OW & 6.955208 & -15.356535 & -17.988417 \\
\hline HW & 6.463901 & -16.131664 & -18.385648 \\
\hline HW & 7.826125 & -15.234581 & -18.464474 \\
\hline OW & 2.603040 & 13.694545 & -12.645357 \\
\hline HW & 2.749995 & 12.862415 & -13.180114 \\
\hline
\end{tabular}




\begin{tabular}{|c|c|c|c|}
\hline HW & 1.728989 & 13.631191 & -12.163671 \\
\hline OW & -2.921257 & 14.609652 & -12.674573 \\
\hline HW & -3.581243 & 14.165576 & -12.068590 \\
\hline HW & -2.881980 & 15.586816 & -12.465748 \\
\hline OW & -2.325816 & -11.917357 & -16.819020 \\
\hline HW & -1.887614 & -11.771689 & -17.706015 \\
\hline HW & -2.410097 & -12.898669 & -16.646036 \\
\hline OW & 6.571355 & -11.214943 & 19.995563 \\
\hline HW & 6.240496 & -10.642904 & 20.746099 \\
\hline HW & 7.456045 & -10.871843 & 19.679960 \\
\hline OW & -2.021283 & 9.182808 & 4.397725 \\
\hline HW & -2.956188 & 8.842138 & 4.497214 \\
\hline HW & -2.002202 & 10.164378 & 4.587870 \\
\hline OW & -6.768067 & 17.870327 & 15.879276 \\
\hline HW & -6.327481 & 17.762925 & 16.770538 \\
\hline HW & -7.683265 & -17.626421 & 16.003900 \\
\hline OW & 2.902597 & 15.275382 & -21.430739 \\
\hline HW & 3.695376 & 15.360387 & -20.827186 \\
\hline HW & 3.186843 & 15.426059 & -22.377576 \\
\hline OW & -0.190690 & 16.702961 & -12.951814 \\
\hline HW & 0.790196 & 16.861927 & -13.064032 \\
\hline HW & -0.424005 & 16.731989 & -11.979846 \\
\hline OW & 0.924432 & 8.817707 & -10.494741 \\
\hline HW & 0.510582 & 9.089770 & -11.363482 \\
\hline HW & 1.920011 & 8.808513 & -10.588221 \\
\hline OW & 7.569751 & 12.352541 & 15.548970 \\
\hline HW & 7.592697 & 13.332161 & 15.349420 \\
\hline HW & 8.354166 & 11.906194 & 15.118312 \\
\hline OW & 6.672476 & 3.661869 & 7.507394 \\
\hline HW & 7.299704 & 3.054247 & 7.020176 \\
\hline HW & 7.130571 & 4.529956 & 7.698632 \\
\hline OW & -9.071803 & 7.149201 & 22.401140 \\
\hline HW & -8.333225 & 7.314742 & 23.054667 \\
\hline HW & -9.701954 & 6.468978 & 22.775581 \\
\hline OW & -3.069847 & 9.190973 & 13.274813 \\
\hline HW & -2.689382 & 8.303761 & 13.013851 \\
\hline HW & -2.979073 & 9.314942 & 14.262938 \\
\hline OW & 7.272832 & -3.871353 & -20.870179 \\
\hline HW & 6.573053 & -4.346505 & -21.403602 \\
\hline HW & 7.164122 & -2.883647 & -20.982517 \\
\hline OW & -8.502113 & 4.890340 & 14.816325 \\
\hline HW & -8.428357 & 4.447826 & 15.710049 \\
\hline HW & -8.248294 & 5.854003 & 14.899573 \\
\hline OW & 5.917150 & -7.714817 & -10.672717 \\
\hline HW & 6.006824 & -8.387008 & -11.407643 \\
\hline HW & 6.824618 & -7.392461 & -10.403296 \\
\hline OW & -3.959483 & 11.011681 & -4.008795 \\
\hline HW & -4.524453 & 11.817072 & -3.829476 \\
\hline HW & -3.933500 & 10.434352 & -3.192698 \\
\hline OW & -9.397738 & -16.519879 & 3.557172 \\
\hline HW & -8.774959 & -15.846695 & 3.158461 \\
\hline HW & -9.526382 & -16.320620 & 4.528638 \\
\hline
\end{tabular}




\begin{tabular}{|c|c|c|c|}
\hline OW & -0.237201 & -10.897720 & -3.745142 \\
\hline HW & 0.251878 & -10.132043 & -3.327362 \\
\hline HW & -0.503323 & -10.654160 & -4.677803 \\
\hline OW & 2.772402 & -9.939937 & -20.942328 \\
\hline HW & 3.231632 & -10.810818 & -21.117465 \\
\hline HW & 2.853603 & -9.352467 & -21.747489 \\
\hline OW & 6.068012 & -1.705509 & -3.914566 \\
\hline HW & 5.254924 & -1.124433 & -3.949749 \\
\hline HW & 5.806790 & -2.627301 & -3.628098 \\
\hline OW & 4.399085 & 2.018278 & 2.258159 \\
\hline HW & 4.360626 & 2.918367 & 1.824153 \\
\hline HW & 5.337886 & 1.825832 & 2.543845 \\
\hline OW & -3.884510 & 4.090673 & -2.467491 \\
\hline HW & -3.093621 & 4.396118 & -2.997772 \\
\hline HW & -4.639943 & 4.733276 & -2.595481 \\
\hline OW & -6.881150 & -3.314558 & 0.607028 \\
\hline HW & -5.946717 & -3.452321 & 0.278615 \\
\hline HW & -6.870434 & -3.186545 & 1.598742 \\
\hline OW & 4.390331 & -14.584897 & 19.360218 \\
\hline HW & 3.432396 & -14.745078 & 19.122094 \\
\hline HW & 4.502185 & -14.647311 & 20.351980 \\
\hline OW & 0.274110 & 2.558809 & 20.201298 \\
\hline HW & 0.874607 & 3.354468 & 20.121733 \\
\hline HW & -0.525603 & 2.798497 & 20.751760 \\
\hline OW & 8.384868 & 4.909402 & 2.799153 \\
\hline HW & 9.254399 & 5.360209 & 2.597438 \\
\hline HW & 7.808240 & 4.916692 & 1.982178 \\
\hline OW & 6.883058 & -12.677387 & 7.558430 \\
\hline HW & 6.092732 & -13.098814 & 8.003161 \\
\hline HW & 7.679971 & -12.747816 & 8.158404 \\
\hline OW & 3.039278 & -13.041380 & 3.613417 \\
\hline HW & 3.177331 & -13.416520 & 4.530047 \\
\hline HW & 2.143583 & -12.599286 & 3.565619 \\
\hline OW & -7.062028 & -3.078338 & 3.365254 \\
\hline HW & -7.739318 & -2.398994 & 3.647693 \\
\hline HW & -6.401411 & -3.217095 & 4.103042 \\
\hline OW & -3.187019 & -7.794938 & 11.671168 \\
\hline HW & -4.049538 & -7.810016 & 11.165368 \\
\hline HW & -3.326389 & -8.189142 & 12.579562 \\
\hline OW & -2.828420 & -13.603384 & -6.610786 \\
\hline HW & -3.290253 & -14.000346 & -7.403964 \\
\hline HW & -2.549783 & -14.333826 & -5.987235 \\
\hline OW & -4.123375 & 5.628630 & 2.972317 \\
\hline HW & -3.893831 & 6.571178 & 2.729599 \\
\hline HW & -4.120222 & 5.062043 & 2.148321 \\
\hline OW & 1.111363 & -13.558661 & -21.219884 \\
\hline HW & 0.907434 & -13.744045 & -22.181157 \\
\hline HW & 0.440323 & -12.913081 & -20.855286 \\
\hline OW & 0.433560 & 8.734015 & 19.939592 \\
\hline HW & -0.205340 & 9.410120 & 19.572593 \\
\hline HW & -0.033919 & 8.167749 & 20.618421 \\
\hline OW & -9.424283 & 9.139281 & 15.867110 \\
\hline
\end{tabular}




\begin{tabular}{|c|c|c|c|}
\hline HW & -9.195823 & 9.332725 & 16.821252 \\
\hline HW & -8.729761 & 8.536966 & 15.473595 \\
\hline OW & 8.454499 & 3.442969 & -3.379028 \\
\hline HW & 8.135112 & 2.550238 & -3.061184 \\
\hline HW & 7.844006 & 3.779474 & -4.096010 \\
\hline OW & -8.180385 & -12.591495 & -21.690631 \\
\hline HW & -8.451388 & -12.362828 & -22.625654 \\
\hline HW & -7.352746 & -13.152379 & -21.711202 \\
\hline OW & 9.018725 & 15.144634 & -14.782744 \\
\hline HW & 8.787862 & 15.258504 & -15.749044 \\
\hline HW & 9.988172 & 15.347921 & -14.645465 \\
\hline OW & 0.481994 & -5.629138 & 4.227141 \\
\hline HW & -0.480808 & -5.847991 & 4.385623 \\
\hline HW & 1.019140 & -6.472614 & 4.222441 \\
\hline OW & -1.759936 & -6.227898 & -7.012878 \\
\hline HW & -1.708021 & -6.096852 & -6.022862 \\
\hline HW & -2.718784 & -6.265937 & -7.294237 \\
\hline OW & -1.876610 & 1.661612 & 10.121972 \\
\hline HW & -1.351826 & 2.237840 & 9.495422 \\
\hline HW & -1.470719 & 0.748249 & 10.153913 \\
\hline OW & 4.379622 & -7.549946 & -4.431292 \\
\hline HW & 3.784456 & -7.845088 & -5.178734 \\
\hline HW & 4.197070 & -8.105985 & -3.620431 \\
\hline OW & 2.113175 & -2.250456 & -4.305665 \\
\hline HW & 2.235036 & -3.232131 & -4.159162 \\
\hline HW & 2.046111 & -2.065569 & -5.286134 \\
\hline OW & -5.668278 & 13.305251 & -3.288527 \\
\hline HW & -5.625835 & 13.705447 & -2.373080 \\
\hline HW & -5.487838 & 14.013793 & -3.970737 \\
\hline OW & -1.460513 & 8.608951 & -9.056120 \\
\hline HW & -1.290647 & 8.964625 & -8.137076 \\
\hline HW & -0.589201 & 8.477033 & -9.528785 \\
\hline OW & 8.163476 & -4.446849 & 10.153532 \\
\hline HW & 8.241916 & -4.493446 & 9.157703 \\
\hline HW & 8.421367 & -3.532519 & 10.465771 \\
\hline OW & 8.384274 & 7.417319 & -4.053267 \\
\hline HW & 7.805974 & 7.832126 & -4.755766 \\
\hline HW & 8.993589 & 8.111682 & -3.670389 \\
\hline OW & 6.115275 & -12.732343 & -13.646240 \\
\hline HW & 6.563748 & -12.575804 & -14.526222 \\
\hline HW & 5.398275 & -12.048470 & -13.511224 \\
\hline OW & -6.747619 & -8.092236 & -9.893825 \\
\hline HW & -7.461092 & -7.903339 & -9.219086 \\
\hline HW & -6.064785 & -8.704024 & -9.494508 \\
\hline OW & -3.868983 & -2.090149 & -10.764667 \\
\hline HW & -3.087067 & -2.302610 & -10.178606 \\
\hline HW & -3.775188 & -1.160744 & -11.121610 \\
\hline OW & -9.690454 & -1.941417 & 11.572147 \\
\hline HW & 10.057834 & -1.705525 & 11.583570 \\
\hline HW & -9.400027 & -2.112459 & 10.630660 \\
\hline OW & 2.324190 & 6.941788 & -17.244139 \\
\hline HW & 1.665227 & 7.415019 & -17.828793 \\
\hline
\end{tabular}




\begin{tabular}{|c|c|c|c|}
\hline HW & 3.233924 & 6.995614 & -17.655828 \\
\hline $\mathrm{OW}$ & -4.377798 & 16.942257 & 1.310314 \\
\hline HW & -4.408083 & 17.934024 & 1.434738 \\
\hline HW & -5.286787 & 16.609993 & 1.058639 \\
\hline $\mathrm{OW}$ & 5.472399 & 3.495652 & -17.819063 \\
\hline HW & 4.937877 & 4.016714 & -17.153645 \\
\hline HW & 4.949525 & 3.401491 & -18.666256 \\
\hline $\mathrm{OW}$ & -1.325543 & 15.927904 & 17.177870 \\
\hline HW & -1.888530 & 16.724553 & 16.957880 \\
\hline HW & -0.370106 & 16.209323 & 17.266994 \\
\hline OW & 5.126300 & -5.033484 & 13.267789 \\
\hline HW & 4.995374 & -4.861606 & 12.291410 \\
\hline HW & 4.520049 & -5.773020 & 13.560275 \\
\hline $\mathrm{OW}$ & -9.426704 & -3.521440 & -12.503582 \\
\hline HW & -9.008753 & -3.156131 & -11.671797 \\
\hline HW & 10.330495 & -3.253457 & -12.538213 \\
\hline OW & 8.944140 & 9.346484 & 4.949549 \\
\hline HW & 9.942380 & 9.317856 & 5.001498 \\
\hline HW & 8.657394 & 9.259501 & 3.995499 \\
\hline OW & -7.880511 & -6.277410 & 8.174549 \\
\hline HW & -8.687164 & -6.563700 & 8.691606 \\
\hline HW & -7.200391 & -7.010435 & 8.185091 \\
\hline $\mathrm{OW}$ & 3.102926 & -14.440106 & 15.420778 \\
\hline HW & 3.955949 & -13.919810 & 15.380252 \\
\hline HW & 3.309465 & -15.418482 & 15.431896 \\
\hline OW & -9.897476 & 12.376716 & 18.768332 \\
\hline HW & 10.263619 & 13.189096 & 18.602006 \\
\hline HW & -9.679339 & 12.315920 & 19.742354 \\
\hline OW & -2.666343 & 10.105469 & 9.278291 \\
\hline $\mathrm{HW}$ & -3.035011 & 9.260451 & 9.665627 \\
\hline HW & -1.667442 & 10.082948 & 9.319389 \\
\hline OW & -6.870839 & 16.490828 & 0.003156 \\
\hline HW & -7.657315 & 16.183665 & 0.538979 \\
\hline HW & -6.962931 & 17.466739 & -0.194628 \\
\hline $\mathrm{OW}$ & 0.156472 & 13.437138 & -11.529338 \\
\hline HW & 0.286514 & 13.010393 & -10.634364 \\
\hline HW & -0.318608 & 14.309831 & -11.416614 \\
\hline OW & 0.320522 & 5.841447 & 16.833029 \\
\hline HW & 0.980695 & 5.207140 & 16.430752 \\
\hline HW & 0.798365 & 6.485165 & 17.430769 \\
\hline OW & 8.034930 & 2.895673 & -15.483892 \\
\hline HW & 8.857415 & 3.120264 & -16.006460 \\
\hline HW & 7.488317 & 2.230321 & -15.992343 \\
\hline OW & 6.929997 & -16.245666 & -14.023058 \\
\hline HW & 6.497307 & -15.705216 & -14.744649 \\
\hline HW & 7.033708 & -15.683693 & -13.202430 \\
\hline OW & 6.921242 & 13.386540 & 11.085825 \\
\hline HW & 7.576570 & 14.141314 & 11.115154 \\
\hline HW & 7.335537 & 12.569632 & 11.487099 \\
\hline OW & 9.293232 & -4.237881 & -18.952426 \\
\hline HW & 8.589559 & -4.404402 & -19.643160 \\
\hline HW & 10.056187 & -3.741660 & -19.366751 \\
\hline
\end{tabular}




\begin{tabular}{|c|c|c|c|}
\hline OW & 8.615685 & -16.796496 & 20.09 \\
\hline HW & 9.052781 & -17.665785 & 20.324460 \\
\hline HW & 7.768403 & -16.700382 & 20.616001 \\
\hline OW & -0.148003 & -5.255812 & 10.284821 \\
\hline HW & 0.200511 & -4.692998 & 11.034340 \\
\hline HW & -0.264550 & -6.197247 & 10.601233 \\
\hline OW & 2.068524 & 11.659163 & 18.626588 \\
\hline HW & 2.305036 & 11.258362 & 19.511698 \\
\hline HW & 2.827846 & 11.530304 & 17.988759 \\
\hline OW & 1.192962 & -11.544156 & -18.697086 \\
\hline HW & 0.749023 & -12.338395 & -18.282234 \\
\hline HW & 2.107538 & -11.432648 & -18.308351 \\
\hline OW & -1.682595 & -17.843755 & -17.437366 \\
\hline HW & -1.040525 & -17.575344 & -18.155490 \\
\hline HW & -2.470904 & 17.578920 & -17.848976 \\
\hline OW & 10.153899 & 17.698494 & -10.209332 \\
\hline HW & 9.906410 & 16.843591 & -9.753383 \\
\hline HW & 9.681292 & -17.419080 & -9.767349 \\
\hline OW & -5.678528 & -13.417477 & -11.296331 \\
\hline HW & -6.524625 & -13.437934 & -11.828967 \\
\hline HW & -4.903475 & -13.258274 & -11.907843 \\
\hline OW & -3.728230 & 0.044124 & 8.218889 \\
\hline HW & -3.572079 & -0.377256 & 9.112229 \\
\hline HW & -2.849451 & 0.221590 & 7.775884 \\
\hline OW & -3.690566 & -3.454038 & -6.326262 \\
\hline HW & -3.909883 & -3.705623 & -5.383604 \\
\hline HW & -4.498691 & -3.580334 & -6.901574 \\
\hline OW & 9.892535 & -8.020966 & 8.382508 \\
\hline HW & -10.112495 & -8.123740 & 9.074069 \\
\hline HW & 9.015254 & -7.853648 & 8.832378 \\
\hline OW & 0.246296 & -15.546193 & -7.665194 \\
\hline HW & 0.830104 & -14.760774 & -7.459558 \\
\hline HW & 0.139032 & -15.631493 & -8.655759 \\
\hline OW & -2.200943 & 4.720080 & -4.780146 \\
\hline HW & -2.154983 & 4.217838 & -5.643651 \\
\hline HW & -2.524688 & 5.650397 & -4.952479 \\
\hline OW & -8.328579 & 12.425213 & -15.025702 \\
\hline HW & -7.331184 & 12.497338 & -15.024156 \\
\hline HW & -8.653981 & 12.323939 & -15.965839 \\
\hline OW & 5.661117 & -12.588800 & 16.153019 \\
\hline HW & 6.581198 & -12.206289 & 16.237490 \\
\hline HW & 5.096907 & -12.271361 & 16.915187 \\
\hline OW & -6.790551 & 13.203974 & 10.949650 \\
\hline HW & -6.240527 & 13.004119 & 10.138766 \\
\hline HW & -7.084682 & 12.346812 & 11.372450 \\
\hline OW & -0.084927 & 8.829158 & -21.711486 \\
\hline HW & 0.270663 & 8.552451 & -22.604229 \\
\hline HW & -1.030172 & 8.516549 & -21.617736 \\
\hline OW & -7.207967 & 4.174438 & 12.495177 \\
\hline HW & -6.425606 & 3.551900 & 12.476277 \\
\hline HW & -7.591871 & 4.199363 & 13.418214 \\
\hline OW & 9.031658 & 0.912219 & -13.450971 \\
\hline
\end{tabular}




\begin{tabular}{|c|c|c|c|}
\hline HW & 9.388409 & 1.436241 & -12.677583 \\
\hline HW & 9.161434 & 1.433049 & -14.294710 \\
\hline OW & -5.199244 & -11.004665 & -16.465172 \\
\hline HW & -5.637102 & -10.449968 & -17.172697 \\
\hline HW & -4.249754 & -11.184270 & -16.722484 \\
\hline OW & -7.972519 & 1.728478 & 16.357197 \\
\hline HW & -7.715513 & 2.676962 & 16.542468 \\
\hline HW & -8.943931 & 1.601143 & 16.557558 \\
\hline OW & 5.010632 & -16.327035 & -0.358799 \\
\hline HW & 5.406894 & -16.236559 & -1.272468 \\
\hline HW & 4.742667 & -15.424817 & -0.020867 \\
\hline OW & -2.894701 & -16.357814 & 8.032471 \\
\hline HW & -2.060275 & -16.908440 & 8.009141 \\
\hline HW & -3.691242 & -16.961188 & 8.070708 \\
\hline OW & 5.927235 & 15.017474 & -14.848942 \\
\hline HW & 6.491568 & 14.409385 & -14.290591 \\
\hline HW & 5.249349 & 14.477956 & -15.348332 \\
\hline OW & -3.705685 & 14.504496 & 12.654275 \\
\hline HW & -4.543122 & 14.849168 & 13.078425 \\
\hline HW & -3.771815 & 13.512829 & 12.543717 \\
\hline OW & 7.395600 & -14.514102 & 5.631017 \\
\hline HW & 7.412375 & -15.404559 & 6.085776 \\
\hline HW & 7.137943 & -13.807867 & 6.290445 \\
\hline OW & -9.002646 & 13.681709 & -0.213765 \\
\hline HW & -9.889914 & 13.254601 & -0.039596 \\
\hline HW & -8.276716 & 13.005692 & -0.087171 \\
\hline OW & -3.291581 & 14.848424 & -8.570295 \\
\hline HW & -4.198409 & 15.172553 & -8.300845 \\
\hline HW & -3.172487 & 14.972228 & -9.555429 \\
\hline OW & -10.068170 & -8.685895 & -13.096173 \\
\hline HW & -10.324486 & -8.697663 & -14.062695 \\
\hline HW & -9.105969 & -8.427670 & -13.009629 \\
\hline OW & -1.380309 & 15.255600 & -6.785322 \\
\hline HW & -1.123132 & 16.209190 & -6.941932 \\
\hline HW & -2.228766 & 15.054486 & -7.274889 \\
\hline OW & 5.180507 & -15.135983 & -15.891671 \\
\hline HW & 4.598146 & -14.470506 & -15.424772 \\
\hline HW & 5.554733 & -14.725836 & -16.723377 \\
\hline OW & 6.056580 & 5.905364 & 12.822665 \\
\hline HW & 7.011110 & 6.110389 & 12.606246 \\
\hline HW & 5.457409 & 6.442350 & 12.228830 \\
\hline OW & -9.030712 & -0.363230 & -14.291934 \\
\hline HW & -9.907590 & -0.737237 & -13.989933 \\
\hline HW & -8.601948 & 0.132537 & -13.536701 \\
\hline OW & -5.666349 & -7.712433 & 18.106504 \\
\hline HW & -5.729676 & -8.451036 & 18.777664 \\
\hline HW & -5.786443 & -8.086488 & 17.186907 \\
\hline OW & 8.549807 & 2.042761 & 6.187566 \\
\hline HW & 9.028972 & 1.276961 & 6.616462 \\
\hline HW & 8.683569 & 2.007035 & 5.197197 \\
\hline OW & -3.831259 & 7.341843 & -7.533891 \\
\hline HW & -3.037326 & 7.572382 & -8.096494 \\
\hline
\end{tabular}




\begin{tabular}{|c|c|c|c|}
\hline HW & -4.660006 & 7.389330 & -8.091496 \\
\hline OW & -3.692215 & 12.643168 & -19.500391 \\
\hline HW & -2.741425 & 12.764444 & -19.785508 \\
\hline HW & -3.787460 & 11.771430 & -19.019765 \\
\hline OW & -8.219603 & 15.079443 & 21.422661 \\
\hline HW & -7.285590 & 15.087738 & 21.065517 \\
\hline HW & -8.640817 & 15.974329 & 21.275149 \\
\hline OW & -5.937688 & 16.045531 & 13.687542 \\
\hline HW & -6.387376 & 16.553191 & 14.422432 \\
\hline HW & -6.315564 & 16.329782 & 12.806400 \\
\hline OW & -4.064181 & -13.033895 & 2.434358 \\
\hline HW & -5.010277 & -13.288595 & 2.634432 \\
\hline HW & -3.938698 & -12.969006 & 1.444387 \\
\hline OW & -6.133457 & 17.114130 & 5.626243 \\
\hline HW & -6.974106 & 17.001986 & 5.096400 \\
\hline HW & -5.349227 & 17.137108 & 5.006198 \\
\hline OW & -6.477130 & 11.795120 & 13.488602 \\
\hline HW & -5.695782 & 11.862403 & 12.868143 \\
\hline HW & -6.604172 & 10.841771 & 13.762438 \\
\hline OW & -1.690398 & -13.367554 & -3.652552 \\
\hline HW & -1.239817 & -12.482838 & -3.533157 \\
\hline HW & -1.123497 & -13.955094 & -4.229978 \\
\hline OW & -2.863941 & 10.147774 & -18.501190 \\
\hline HW & -2.952114 & 9.331158 & -19.071597 \\
\hline HW & -1.895925 & 10.369757 & -18.384282 \\
\hline OW & -6.484702 & -11.278317 & 18.845776 \\
\hline $\mathrm{HW}$ & -6.923673 & -11.416758 & 17.958005 \\
\hline HW & -7.149333 & -11.440448 & 19.575145 \\
\hline $\mathrm{OW}$ & 4.003597 & -11.217681 & -9.847836 \\
\hline HW & 4.199443 & -10.425461 & -9.269880 \\
\hline HW & 3.034093 & -11.451741 & -9.775188 \\
\hline OW & -9.226142 & 16.146393 & -6.677567 \\
\hline HW & -9.660851 & 17.043974 & -6.604249 \\
\hline HW & -9.015012 & 15.955607 & -7.636225 \\
\hline OW & -9.113486 & -7.877621 & 14.317794 \\
\hline HW & -9.342638 & -8.144915 & 15.253766 \\
\hline HW & -9.937343 & -7.554177 & 13.852345 \\
\hline OW & -9.839861 & 4.270994 & -7.875619 \\
\hline HW & -9.258235 & 3.661462 & -8.414306 \\
\hline HW & 9.925901 & 4.148299 & -8.148337 \\
\hline $\mathrm{OW}$ & -0.339729 & 11.129118 & 5.680163 \\
\hline HW & 0.207134 & 10.490740 & 6.221839 \\
\hline HW & -0.073870 & 11.062938 & 4.718426 \\
\hline OW & -0.328793 & 12.764935 & -6.754047 \\
\hline HW & -1.134308 & 12.180639 & -6.655327 \\
\hline HW & -0.610773 & 13.724355 & -6.752696 \\
\hline OW & 9.908735 & -6.268322 & 6.231154 \\
\hline HW & 9.829806 & -6.968998 & 6.940254 \\
\hline $\mathrm{HW}$ & -10.223002 & -6.603617 & 5.495270 \\
\hline OW & 1.500059 & -3.020372 & -9.297241 \\
\hline HW & 1.015087 & -2.497399 & -8.596312 \\
\hline HW & 0.931936 & -3.084989 & -10.117644 \\
\hline
\end{tabular}




\begin{tabular}{|c|c|c|c|}
\hline OW & 10.348661 & -9.295845 & -4.992027 \\
\hline HW & 9.530607 & -9.276714 & -4.417203 \\
\hline HW & -9.750227 & -10.007735 & -4.664257 \\
\hline OW & -0.123329 & 16.357202 & -10.343403 \\
\hline HW & -0.372657 & 17.210838 & -9.886086 \\
\hline HW & 0.746333 & 16.025088 & -9.978180 \\
\hline OW & 1.322996 & -0.065153 & -13.838477 \\
\hline HW & 1.056978 & -0.947830 & -14.225927 \\
\hline HW & 1.033311 & -0.019890 & -12.882426 \\
\hline OW & 6.185345 & -11.412553 & -5.267805 \\
\hline HW & 5.803005 & -11.920602 & -6.039623 \\
\hline HW & 7.017654 & -11.866380 & -4.949525 \\
\hline OW & -6.196284 & 7.285100 & 0.171743 \\
\hline HW & -6.046244 & 6.490203 & -0.416158 \\
\hline HW & -6.916389 & 7.858004 & -0.219702 \\
\hline OW & 7.214375 & -9.224255 & 17.063848 \\
\hline HW & 7.794414 & -8.930666 & 17.823690 \\
\hline HW & 7.536998 & -10.105955 & 16.719580 \\
\hline OW & -7.212814 & 1.633285 & -15.967376 \\
\hline HW & -7.149510 & 1.060254 & -16.784461 \\
\hline HW & -7.299352 & 1.051860 & -15.158391 \\
\hline OW & 9.520164 & 16.284553 & -1.253236 \\
\hline HW & 10.350086 & 15.820125 & -1.562325 \\
\hline HW & 9.216090 & 15.883709 & -0.389023 \\
\hline OW & -4.684913 & -0.466109 & 1.233106 \\
\hline HW & -5.048306 & -0.166237 & 0.351050 \\
\hline HW & -5.157520 & 0.017223 & 1.970013 \\
\hline OW & -3.430934 & -6.667786 & -18.532202 \\
\hline HW & -3.552935 & -7.080229 & -17.629424 \\
\hline HW & -4.271577 & -6.196998 & -18.799930 \\
\hline OW & 9.181541 & 6.372806 & -18.190574 \\
\hline HW & 9.849934 & 5.901828 & -17.614873 \\
\hline HW & 8.527131 & 5.709060 & -18.552775 \\
\hline OW & -4.291101 & 16.173438 & -3.423298 \\
\hline HW & -5.155937 & 16.674745 & -3.450713 \\
\hline HW & -3.837946 & 16.338226 & -2.547230 \\
\hline OW & 3.827836 & -8.514502 & -8.879525 \\
\hline HW & 2.933858 & -8.545478 & -9.326564 \\
\hline HW & 4.518478 & -8.211242 & -9.536067 \\
\hline OW & -4.238142 & -14.646056 & -8.716620 \\
\hline HW & -3.601409 & -14.980346 & -9.411473 \\
\hline HW & -4.843305 & -13.960483 & -9.121301 \\
\hline OW & 3.540849 & 8.903945 & 16.030258 \\
\hline HW & 3.201656 & 9.632359 & 15.434975 \\
\hline HW & 2.846959 & 8.683446 & 16.715748 \\
\hline OW & 8.995494 & 13.083025 & 2.258447 \\
\hline HW & 8.171986 & 12.519684 & 2.325384 \\
\hline HW & 8.744430 & 14.007694 & 1.972169 \\
\hline OW & -3.623717 & 4.255182 & 18.543098 \\
\hline HW & -2.987030 & 4.096731 & 17.788430 \\
\hline HW & -3.108925 & 4.424606 & 19.383505 \\
\hline OW & -9.979256 & 6.072784 & -10.294642 \\
\hline
\end{tabular}




\begin{tabular}{|c|c|c|c|}
\hline HW & -9.556703 & 5.493717 & -10.991873 \\
\hline HW & -10.215191 & 5.516682 & -9.497720 \\
\hline OW & -0.308908 & -17.002689 & -0.671885 \\
\hline HW & -1.048737 & -16.377561 & -0.920620 \\
\hline HW & -0.085874 & -17.583447 & -1.454814 \\
\hline OW & -5.551637 & -2.945667 & -15.619803 \\
\hline HW & -6.378544 & -3.341773 & -15.220648 \\
\hline HW & -4.751862 & -3.457165 & -15.305589 \\
\hline OW & 6.704076 & -0.146630 & -0.609054 \\
\hline HW & 7.314158 & 0.641079 & -0.523520 \\
\hline HW & 5.793262 & 0.099683 & -0.277772 \\
\hline OW & -5.717853 & -17.543156 & 10.654484 \\
\hline HW & -5.586545 & -17.381788 & 9.676364 \\
\hline HW & -6.289615 & 17.526851 & 10.784867 \\
\hline OW & -0.740607 & 11.938307 & 0.861822 \\
\hline HW & -0.053379 & 11.337207 & 0.453904 \\
\hline HW & -0.305877 & 12.788695 & 1.158215 \\
\hline OW & -2.709487 & 14.096648 & 9.860121 \\
\hline HW & -2.976702 & 14.922606 & 10.356497 \\
\hline HW & -3.507324 & 13.505207 & 9.743269 \\
\hline OW & -8.268315 & 1.979545 & -4.134046 \\
\hline HW & -7.279203 & 2.019179 & -4.275772 \\
\hline HW & -8.563702 & 2.784056 & -3.618771 \\
\hline OW & -9.587997 & 8.100908 & -19.943648 \\
\hline HW & -9.342516 & 7.142846 & -20.091491 \\
\hline HW & -8.890919 & 8.690404 & -20.351786 \\
\hline $\mathrm{OW}$ & -0.667759 & -4.425506 & -11.497858 \\
\hline HW & -0.316197 & -3.773718 & -12.169853 \\
\hline HW & -0.272273 & -5.327700 & -11.670014 \\
\hline $\mathrm{OW}$ & -7.088160 & 8.592569 & -14.307289 \\
\hline HW & -6.101370 & 8.739383 & -14.375781 \\
\hline $\mathrm{HW}$ & -7.482070 & 8.541723 & -15.225030 \\
\hline OW & 7.730263 & 1.096405 & 9.657138 \\
\hline HW & 7.679533 & 1.690834 & 10.459685 \\
\hline HW & 8.665964 & 1.091801 & 9.304375 \\
\hline OW & 4.302246 & 2.869618 & -20.182778 \\
\hline HW & 3.367785 & 3.223641 & -20.220849 \\
\hline HW & 4.401682 & 2.120189 & -20.837354 \\
\hline OW & -2.914394 & 0.560002 & 18.846090 \\
\hline HW & -2.936579 & 0.435760 & 19.838094 \\
\hline HW & -2.060135 & 1.009837 & 18.585534 \\
\hline OW & 3.747301 & 13.787142 & -16.165748 \\
\hline HW & 3.932470 & 13.483909 & -17.100501 \\
\hline HW & 2.765958 & 13.943894 & -16.054418 \\
\hline OW & -4.964894 & -9.623402 & -8.398145 \\
\hline HW & -4.916719 & -10.164467 & -7.558545 \\
\hline HW & -4.371950 & -10.031636 & -9.092237 \\
\hline OW & 6.794119 & 11.422522 & 2.300723 \\
\hline HW & 6.499100 & 11.204393 & 1.370463 \\
\hline HW & 6.794266 & 10.589698 & 2.854260 \\
\hline OW & 1.383049 & -2.120778 & 20.532031 \\
\hline HW & 0.913388 & -1.835869 & 19.696421 \\
\hline
\end{tabular}




\begin{tabular}{|c|c|c|c|}
\hline HW & 1.582715 & -1.317166 & 21.092693 \\
\hline OW & -6.199635 & -9.100287 & 20.795153 \\
\hline HW & -6.600094 & -10.016488 & 20.809613 \\
\hline HW & -5.324183 & -9.111510 & 21.278329 \\
\hline OW & 5.155640 & -8.895637 & -19.770408 \\
\hline HW & 4.456444 & -9.434320 & -20.240456 \\
\hline HW & 5.640718 & -9.476721 & -19.116923 \\
\hline OW & 4.262753 & 4.798220 & 1.412902 \\
\hline HW & 3.740327 & 5.136518 & 0.630198 \\
\hline HW & 3.853978 & 5.139769 & 2.259216 \\
\hline OW & 6.319884 & 2.863636 & 20.750748 \\
\hline HW & 5.976008 & 1.967717 & 20.469537 \\
\hline HW & 7.128678 & 2.744474 & 21.326641 \\
\hline OW & -7.275481 & 12.826964 & -12.333633 \\
\hline HW & -7.116664 & 12.459648 & -13.250070 \\
\hline HW & -7.910605 & 12.231706 & -11.841409 \\
\hline OW & -6.290463 & -1.949222 & -5.194084 \\
\hline HW & -7.178560 & -1.697012 & -4.809800 \\
\hline HW & -5.584671 & -1.855398 & -4.491905 \\
\hline OW & 8.217717 & -6.203895 & -10.066224 \\
\hline HW & 8.902787 & -6.786580 & -10.503440 \\
\hline HW & 8.674245 & -5.451902 & -9.590735 \\
\hline OW & 7.026277 & 11.334292 & -16.535731 \\
\hline HW & 6.089597 & 10.984836 & -16.513092 \\
\hline HW & 7.531434 & 10.985208 & -15.746454 \\
\hline OW & -3.530192 & 9.709919 & -10.570096 \\
\hline $\mathrm{HW}$ & -2.808909 & 9.185398 & -10.117737 \\
\hline HW & -3.949890 & 10.335453 & -9.912403 \\
\hline $\mathrm{OW}$ & 1.693044 & -17.479307 & -5.291481 \\
\hline HW & 1.415454 & -16.519776 & -5.244092 \\
\hline HW & 2.209539 & -17.635678 & -6.133372 \\
\hline OW & 7.058259 & 16.722881 & -2.397569 \\
\hline HW & 6.635058 & 15.837856 & -2.203580 \\
\hline HW & 7.975460 & 16.748065 & -1.999941 \\
\hline OW & 3.752732 & 3.595033 & 21.866189 \\
\hline HW & 4.700318 & 3.544429 & 21.550721 \\
\hline HW & 3.737648 & 3.701066 & 22.860437 \\
\hline OW & -5.161973 & 7.141253 & -21.475480 \\
\hline HW & -6.159224 & 7.189576 & -21.531645 \\
\hline HW & -4.799644 & 6.742137 & -22.317753 \\
\hline $\mathrm{OW}$ & 10.354672 & 1.492028 & 0.103556 \\
\hline HW & -9.633557 & 1.513526 & 0.784768 \\
\hline HW & 10.030253 & 0.552667 & -0.007596 \\
\hline OW & -7.977826 & 1.318222 & -12.253227 \\
\hline HW & -8.486992 & 2.176510 & -12.189251 \\
\hline $\mathrm{HW}$ & -8.356249 & 0.657092 & -11.605382 \\
\hline OW & -4.531401 & 11.640338 & 18.313508 \\
\hline HW & -5.080601 & 12.402406 & 17.970533 \\
\hline $\mathrm{HW}$ & -4.765926 & 11.464208 & 19.269529 \\
\hline OW & 6.808638 & -8.641546 & -14.030040 \\
\hline HW & 7.184577 & -8.790320 & -14.944664 \\
\hline HW & 7.513840 & -8.826697 & -13.345636 \\
\hline
\end{tabular}




\begin{tabular}{|c|c|c|c|}
\hline OW & 8.556861 & 7.694307 & 12.648848 \\
\hline HW & 8.374894 & 7.603791 & 13.627978 \\
\hline HW & 8.403292 & 8.641532 & 12.367457 \\
\hline OW & 3.581344 & 14.977739 & -3.057510 \\
\hline HW & 3.680178 & 15.913863 & -3.395007 \\
\hline HW & 3.296058 & 14.997274 & -2.099267 \\
\hline OW & -10.326350 & 0.712091 & 9.562675 \\
\hline HW & -9.409008 & 1.078352 & 9.718678 \\
\hline HW & -10.261838 & -0.255439 & 9.318292 \\
\hline OW & 2.732052 & -5.300905 & -6.501689 \\
\hline HW & 2.600267 & -5.444324 & -7.482537 \\
\hline HW & 3.251905 & -4.459379 & -6.354761 \\
\hline OW & 8.190107 & 5.907228 & 7.541873 \\
\hline HW & 8.839161 & 5.518583 & 6.887897 \\
\hline HW & 8.648510 & 6.056023 & 8.418073 \\
\hline OW & 0.202321 & 10.265594 & 9.800888 \\
\hline HW & 1.065986 & 10.622657 & 9.445095 \\
\hline HW & 0.347183 & 9.346264 & 10.166738 \\
\hline OW & -0.818920 & -10.047155 & -0.355897 \\
\hline HW & -0.472590 & -9.594755 & 0.465923 \\
\hline HW & -0.131480 & -10.687376 & -0.698744 \\
\hline $\mathrm{OW}$ & 2.764707 & -3.712746 & 1.965043 \\
\hline HW & 2.553822 & -4.560726 & 1.478774 \\
\hline HW & 3.551773 & -3.269434 & 1.536088 \\
\hline OW & -0.516832 & -14.979994 & 13.514137 \\
\hline HW & -0.425949 & -14.571079 & 12.606102 \\
\hline $\mathrm{HW}$ & -1.215623 & -15.694891 & 13.489328 \\
\hline OW & 2.229212 & -13.736385 & -7.237945 \\
\hline HW & 2.552356 & -13.014964 & -6.625467 \\
\hline HW & 1.858531 & -13.325305 & -8.070777 \\
\hline OW & 7.909657 & 10.442779 & -14.255172 \\
\hline HW & 8.900194 & 10.388867 & -14.128958 \\
\hline HW & 7.472496 & 9.669846 & -13.795317 \\
\hline OW & 3.353848 & -6.523494 & -11.980519 \\
\hline HW & 4.087651 & -7.178190 & -11.799114 \\
\hline HW & 3.436638 & -6.180891 & -12.916344 \\
\hline OW & -5.294415 & 11.146370 & 20.955120 \\
\hline HW & -4.811872 & 11.457520 & 21.773862 \\
\hline HW & -6.276454 & 11.110628 & 21.140385 \\
\hline OW & -1.763269 & 4.323024 & 16.656367 \\
\hline HW & -1.070423 & 5.031872 & 16.788656 \\
\hline HW & -1.412013 & 3.447541 & 16.988254 \\
\hline OW & 1.983007 & -0.504659 & 8.586973 \\
\hline HW & 2.660697 & 0.230688 & 8.586368 \\
\hline HW & 1.638025 & -0.640764 & 7.658285 \\
\hline OW & 7.113133 & -12.469733 & -1.926349 \\
\hline HW & 7.424675 & -13.406048 & -2.088385 \\
\hline HW & 6.503729 & -12.453699 & -1.133651 \\
\hline $\mathrm{OW}$ & -2.647629 & -12.721419 & -19.695675 \\
\hline HW & -2.704999 & -13.663686 & -19.365763 \\
\hline HW & -3.568871 & -12.342727 & -19.784596 \\
\hline OW & -1.871064 & -16.523217 & 4.487588 \\
\hline
\end{tabular}




\begin{tabular}{|c|c|c|c|}
\hline HW & -2.795285 & -16.293546 & 4.792656 \\
\hline HW & -1.682408 & -16.062992 & 3.620062 \\
\hline OW & -8.562200 & 4.292359 & -3.077767 \\
\hline HW & -9.357398 & 4.207242 & -2.477422 \\
\hline HW & -8.859219 & 4.594621 & -3.983537 \\
\hline OW & 8.198981 & 13.824556 & 7.727145 \\
\hline HW & 7.708204 & 14.683535 & 7.581227 \\
\hline HW & 7.612617 & 13.186220 & 8.225849 \\
\hline OW & -0.435910 & 12.899223 & 10.742282 \\
\hline HW & -1.274985 & 13.324723 & 10.403309 \\
\hline HW & -0.318174 & 12.004627 & 10.311196 \\
\hline OW & -7.420156 & -9.668750 & 2.679376 \\
\hline HW & -8.292008 & -10.143227 & 2.557945 \\
\hline HW & -6.744326 & -10.046062 & 2.046218 \\
\hline OW & -0.689578 & -13.000480 & 1.278213 \\
\hline HW & -0.374763 & -12.717888 & 0.372104 \\
\hline HW & -1.583108 & -12.591360 & 1.463213 \\
\hline OW & 4.515169 & -17.513971 & 4.033010 \\
\hline HW & 4.103483 & -16.852124 & 4.659487 \\
\hline HW & 5.311284 & -17.102427 & 3.589351 \\
\hline OW & 4.274335 & 4.713992 & -4.586398 \\
\hline HW & 3.388758 & 5.075435 & -4.294658 \\
\hline HW & 4.259442 & 3.715331 & -4.536843 \\
\hline OW & 6.862282 & 8.957583 & 20.061149 \\
\hline HW & 6.687083 & 8.340926 & 20.828638 \\
\hline HW & 7.736135 & 9.423355 & 20.200574 \\
\hline OW & 1.082708 & -8.747724 & -2.799552 \\
\hline HW & 0.901980 & -7.956758 & -3.384112 \\
\hline HW & 1.989618 & -8.657712 & -2.387953 \\
\hline OW & 9.291458 & 11.029411 & 7.541127 \\
\hline HW & 9.897781 & 10.235878 & 7.489384 \\
\hline HW & 8.417515 & 10.809963 & 7.107460 \\
\hline OW & 8.082074 & 15.569624 & -5.099602 \\
\hline HW & 7.549300 & 16.023106 & -4.385105 \\
\hline HW & 7.769658 & 15.881386 & -5.996932 \\
\hline OW & 1.831588 & 0.639849 & 21.594845 \\
\hline HW & 1.827689 & 0.872137 & 22.567485 \\
\hline HW & 1.336704 & 1.341731 & 21.082546 \\
\hline OW & -8.714544 & 15.379110 & -2.735863 \\
\hline HW & -8.805430 & 14.548636 & -2.186268 \\
\hline HW & -8.301824 & 15.151608 & -3.617852 \\
\hline OW & 9.089587 & -16.457269 & 12.365756 \\
\hline HW & 8.317700 & -17.086391 & 12.457385 \\
\hline HW & 9.882609 & -16.830295 & 12.847385 \\
\hline OW & 0.009106 & -12.455556 & 6.454713 \\
\hline HW & 0.004357 & -12.747152 & 5.498183 \\
\hline HW & -0.388770 & -13.174704 & 7.024380 \\
\hline OW & -6.682805 & -16.426519 & -20.052427 \\
\hline HW & -6.861622 & -16.071732 & -19.134739 \\
\hline HW & -7.371414 & -17.113621 & -20.284176 \\
\hline OW & -8.322486 & 8.185138 & -5.707806 \\
\hline HW & -7.523137 & 8.766730 & -5.858777 \\
\hline
\end{tabular}




\begin{tabular}{|c|c|c|c|}
\hline HW & -9.086530 & 8.518811 & -6.259982 \\
\hline OW & 6.050275 & 0.277539 & 4.324539 \\
\hline HW & 6.180196 & 0.032024 & 5.285186 \\
\hline HW & 6.857407 & 0.007694 & 3.799447 \\
\hline OW & -0.556374 & 4.689710 & 11.542824 \\
\hline HW & -0.265851 & 3.733271 & 11.514178 \\
\hline HW & -0.071821 & 5.163362 & 12.278257 \\
\hline OW & -1.591133 & -2.008591 & 3.770810 \\
\hline HW & -0.905023 & -2.538874 & 3.272761 \\
\hline HW & -2.363393 & -2.599165 & 4.004982 \\
\hline OW & -3.979071 & -12.382810 & 17.529224 \\
\hline HW & -3.750624 & -13.233343 & 18.002940 \\
\hline HW & -4.714036 & -11.914945 & 18.020071 \\
\hline OW & 6.871807 & -4.988664 & 20.228854 \\
\hline HW & 6.403904 & -5.811002 & 20.552625 \\
\hline HW & 6.367958 & -4.600467 & 19.457206 \\
\hline OW & 8.774968 & -2.974296 & -13.304591 \\
\hline HW & 8.320131 & -2.516953 & -12.540417 \\
\hline $\mathrm{HW}$ & 8.385151 & -2.650386 & -14.166638 \\
\hline OW & 6.445832 & -7.388899 & -1.301067 \\
\hline HW & 6.683552 & -6.689754 & -1.975371 \\
\hline HW & 7.136377 & -8.112087 & -1.313145 \\
\hline OW & -0.266307 & -8.204650 & -22.101302 \\
\hline HW & -0.296762 & -8.526512 & -23.047599 \\
\hline HW & -1.184599 & -7.935321 & -21.811126 \\
\hline OW & 4.777318 & 16.049535 & 5.305212 \\
\hline $\mathrm{HW}$ & 5.166669 & 15.300754 & 4.768804 \\
\hline HW & 4.719818 & 16.871105 & 4.738013 \\
\hline OW & -4.380072 & 8.561039 & -14.897930 \\
\hline HW & -3.968368 & 7.655877 & -15.003667 \\
\hline HW & -3.702641 & 9.193526 & -14.522368 \\
\hline OW & -0.110327 & -1.599301 & 18.294111 \\
\hline HW & -1.090440 & -1.753278 & 18.419287 \\
\hline HW & 0.062308 & -1.295089 & 17.357280 \\
\hline OW & -1.571847 & 15.070576 & 1.343633 \\
\hline HW & -2.340988 & 14.817933 & 0.756612 \\
\hline HW & -1.764170 & 15.946270 & 1.786546 \\
\hline OW & 4.111534 & -8.595219 & -2.126008 \\
\hline HW & 3.702951 & -9.265930 & -1.506971 \\
\hline HW & 4.810460 & -8.073011 & -1.637335 \\
\hline $\mathrm{OW}$ & 9.966020 & -2.457659 & 1.993010 \\
\hline HW & 9.683419 & -3.358972 & 2.321296 \\
\hline HW & -10.033628 & -2.097752 & 2.585934 \\
\hline OW & 2.331179 & 4.083362 & 16.187087 \\
\hline HW & 2.746046 & 4.985455 & 16.068284 \\
\hline HW & 2.657927 & 3.675911 & 17.039859 \\
\hline OW & -5.557511 & -13.029409 & 5.589460 \\
\hline HW & -6.380232 & -13.532115 & 5.854824 \\
\hline $\mathrm{HW}$ & -4.984955 & -13.609331 & 5.009918 \\
\hline OW & -7.863228 & 17.539819 & -17.153622 \\
\hline HW & -7.022321 & -17.799099 & -17.163894 \\
\hline HW & -7.637680 & 16.575973 & -17.011744 \\
\hline
\end{tabular}




\begin{tabular}{|c|c|c|c|}
\hline OW & 5.932018 & 3.189611 & 13.041131 \\
\hline HW & 5.856662 & 2.684112 & 13.900661 \\
\hline HW & 5.676142 & 4.144406 & 13.192438 \\
\hline OW & -8.200949 & 10.162414 & 11.055789 \\
\hline HW & -8.865828 & 10.569234 & 11.682234 \\
\hline HW & -7.418868 & 9.819759 & 11.576306 \\
\hline OW & -4.537793 & -13.287405 & -14.197596 \\
\hline HW & -4.761274 & -12.510964 & -14.786830 \\
\hline HW & -3.828870 & -13.017993 & -13.545794 \\
\hline OW & -8.604753 & 12.686867 & 7.751951 \\
\hline HW & -8.450786 & 11.698791 & 7.752071 \\
\hline HW & -7.724836 & 13.161319 & 7.777281 \\
\hline $\mathrm{OW}$ & -7.830117 & 6.847816 & 4.067400 \\
\hline HW & -7.208575 & 7.284967 & 3.417335 \\
\hline HW & -8.340511 & 7.549753 & 4.564172 \\
\hline OW & -8.167637 & 10.175741 & 8.262070 \\
\hline HW & -8.194336 & 9.191901 & 8.085019 \\
\hline HW & -8.231929 & 10.339109 & 9.246539 \\
\hline OW & -2.474813 & -12.597385 & -12.324846 \\
\hline HW & -1.690507 & -12.032144 & -12.580515 \\
\hline HW & -2.152001 & -13.477314 & -11.976252 \\
\hline OW & -4.814829 & -11.454553 & -6.319387 \\
\hline HW & -4.015444 & -11.983955 & -6.035280 \\
\hline HW & -5.639592 & -12.007963 & -6.203183 \\
\hline OW & 9.665402 & -14.139351 & 15.586020 \\
\hline HW & 9.457423 & -13.218643 & 15.916232 \\
\hline HW & 8.825207 & -14.575667 & 15.263994 \\
\hline OW & -9.454700 & 15.338773 & 6.752812 \\
\hline HW & -9.100321 & 15.855764 & 5.973624 \\
\hline HW & -8.722980 & 15.197451 & 7.419606 \\
\hline $\mathrm{OW}$ & -10.165575 & & -0.141595 \\
\hline HW & -9.960975 & 6.078599 & 0.804699 \\
\hline HW & 10.341147 & 5.504488 & -0.665812 \\
\hline $\mathrm{OW}$ & -2.758835 & 16.297909 & -1.211967 \\
\hline HW & -2.780686 & 16.458393 & -0.225171 \\
\hline $\mathrm{HW}$ & -1.809042 & 16.235487 & -1.518557 \\
\hline $\mathrm{OW}$ & -2.366887 & 10.608221 & -13.223094 \\
\hline HW & -2.882215 & 10.346266 & -12.407118 \\
\hline $\mathrm{HW}$ & -2.179200 & 11.590125 & -13.197822 \\
\hline OW & 2.693120 & -16.998863 & 9.123971 \\
\hline HW & 1.902199 & -17.389213 & 9.595214 \\
\hline $\mathrm{HW}$ & 3.183028 & -16.387438 & 9.745381 \\
\hline OW & -6.297484 & -3.129894 & -18.452232 \\
\hline $\mathrm{HW}$ & -6.407741 & -4.084078 & -18.730398 \\
\hline $\mathrm{HW}$ & -6.285374 & -3.073004 & -17.453925 \\
\hline OW & -9.760076 & -17.193467 & -7.339483 \\
\hline HW & -9.149659 & -16.926421 & -8.085189 \\
\hline $\mathrm{HW}$ & 10.013398 & -17.000925 & -7.598358 \\
\hline $\mathrm{OW}$ & 4.341906 & 14.513656 & 17.757963 \\
\hline HW & 4.901628 & 14.666135 & 18.572495 \\
\hline HW & 4.411323 & 13.555452 & 17.480427 \\
\hline OW & -2.886830 & -6.476107 & 18.329285 \\
\hline
\end{tabular}




\begin{tabular}{|c|c|c|c|}
\hline HW & -1.928813 & -6.212215 & 18.441381 \\
\hline HW & -3.078186 & -7.282299 & 18.889139 \\
\hline OW & 6.416875 & -10.879842 & -18.056653 \\
\hline HW & 7.115579 & -11.017130 & -18.758768 \\
\hline HW & 6.678031 & -10.111537 & -17.472268 \\
\hline OW & -10.006385 & -13.019292 & -19.828729 \\
\hline HW & -9.469704 & -12.727997 & -19.036819 \\
\hline HW & -9.425264 & -13.021775 & -20.642542 \\
\hline OW & -9.638077 & 17.590779 & -3.861364 \\
\hline HW & -9.394713 & 16.728423 & -3.417386 \\
\hline HW & 10.347380 & 17.430529 & -4.520732 \\
\hline OW & -0.155355 & -13.338585 & 10.438314 \\
\hline HW & 0.773829 & -13.501606 & 10.770041 \\
\hline HW & -0.228697 & -13.643945 & 9.488905 \\
\hline OW & 0.819969 & -4.470454 & 7.448167 \\
\hline HW & 0.274774 & -4.597138 & 8.276849 \\
\hline HW & 0.525553 & -5.125327 & 6.752135 \\
\hline OW & 0.682370 & 7.762472 & 10.455403 \\
\hline HW & 1.354617 & 7.140191 & 10.054343 \\
\hline HW & 0.894298 & 7.903808 & 11.422415 \\
\hline OW & 5.478837 & 16.089695 & 21.763636 \\
\hline HW & 6.091982 & 15.557246 & 21.180069 \\
\hline HW & 5.650543 & 15.862739 & 22.722285 \\
\hline OW & 3.416968 & -7.253786 & -18.721855 \\
\hline HW & 2.890773 & -7.012453 & -19.537255 \\
\hline HW & 4.223499 & -7.780893 & -18.989562 \\
\hline OW & -10.059564 & -9.474973 & 21.926146 \\
\hline HW & 10.319302 & -8.902891 & 22.672034 \\
\hline HW & -9.801060 & -8.895616 & 21.153151 \\
\hline OW & -3.029209 & 7.931379 & -19.996622 \\
\hline HW & -3.797020 & 7.682854 & -20.587132 \\
\hline HW & -2.320035 & 7.229083 & -20.058704 \\
\hline OW & -1.623304 & 17.228747 & 21.210075 \\
\hline HW & -2.362436 & 17.220963 & 20.536559 \\
\hline HW & -1.725366 & 16.447577 & 21.825994 \\
\hline OW & -2.588129 & 9.335192 & 15.977797 \\
\hline HW & -3.038812 & 8.760405 & 16.660809 \\
\hline HW & -1.792607 & 8.853903 & 15.609687 \\
\hline OW & 0.103263 & -0.582949 & -4.175865 \\
\hline HW & -0.532830 & -0.905685 & -4.876742 \\
\hline HW & 0.935281 & -1.137163 & -4.200211 \\
\hline OW & 7.738081 & 10.145232 & -3.414464 \\
\hline HW & 7.766149 & 11.108120 & -3.146026 \\
\hline HW & 6.795886 & 9.885577 & -3.626240 \\
\hline OW & 6.285596 & 7.427589 & 3.648556 \\
\hline HW & 6.898246 & 6.676626 & 3.402149 \\
\hline HW & 5.348650 & 7.191651 & 3.390746 \\
\hline OW & 0.612101 & -13.612815 & 15.453631 \\
\hline HW & 1.545666 & -13.930216 & 15.620101 \\
\hline HW & 0.210982 & -14.136625 & 14.702147 \\
\hline OW & 1.961716 & 7.617669 & 1.478388 \\
\hline HW & 2.300011 & 8.500747 & 1.153227 \\
\hline
\end{tabular}




\begin{tabular}{|c|c|c|c|}
\hline HW & 2.220004 & 6.902238 & 0.829199 \\
\hline OW & -2.005250 & 14.675584 & -17.049248 \\
\hline HW & -2.331079 & 13.866465 & -16.560207 \\
\hline HW & -1.407058 & 15.208411 & -16.450698 \\
\hline OW & 5.421684 & -3.327047 & -13.536389 \\
\hline HW & 5.873382 & -3.935502 & -14.188886 \\
\hline HW & 5.159307 & -2.479434 & -13.997595 \\
\hline OW & -9.918532 & -10.872992 & 10.369952 \\
\hline HW & -9.275995 & -11.252414 & 11.035673 \\
\hline HW & -9.434536 & -10.667188 & 9.519427 \\
\hline OW & -1.296316 & -17.751837 & -6.903350 \\
\hline HW & -0.543996 & -17.195517 & -7.256229 \\
\hline HW & -2.103152 & -17.626017 & -7.480571 \\
\hline OW & 2.336761 & -16.842769 & -18.382356 \\
\hline HW & 2.789925 & -16.482629 & -19.197795 \\
\hline HW & 2.619784 & -17.790475 & -18.234875 \\
\hline OW & 3.066676 & 13.328496 & -4.946932 \\
\hline HW & 3.324089 & 13.830880 & -4.121493 \\
\hline HW & 2.240076 & 12.794851 & -4.768168 \\
\hline OW & -2.994440 & -10.696644 & -10.242728 \\
\hline HW & -2.964380 & -11.478292 & -10.865722 \\
\hline HW & -3.145204 & -9.858376 & -10.766729 \\
\hline OW & -4.520967 & -0.484587 & -14.346459 \\
\hline HW & -5.243310 & -1.151746 & -14.528445 \\
\hline HW & -4.789327 & 0.095857 & -13.577649 \\
\hline OW & -8.571578 & 17.345679 & -20.210816 \\
\hline $\mathrm{HW}$ & -8.054578 & 16.556358 & -20.542001 \\
\hline HW & -8.728104 & 17.252858 & -19.227513 \\
\hline $\mathrm{OW}$ & 5.794387 & -5.127419 & -5.198951 \\
\hline HW & 6.365594 & -5.295078 & -6.002452 \\
\hline HW & 5.214510 & -5.924424 & -5.030054 \\
\hline $\mathrm{OW}$ & -8.351526 & 16.802116 & 4.216892 \\
\hline HW & -8.713374 & 16.226261 & 3.483777 \\
\hline HW & -8.711357 & 17.730111 & 4.120219 \\
\hline $\mathrm{OW}$ & -0.596010 & 8.507069 & -3.893288 \\
\hline HW & -1.264744 & 7.792722 & -4.099454 \\
\hline HW & -0.720069 & 8.817833 & -2.950932 \\
\hline OW & 4.401685 & -0.709886 & -15.055254 \\
\hline HW & 5.001070 & -0.025000 & -14.640924 \\
\hline HW & 3.501330 & -0.670787 & -14.621858 \\
\hline $\mathrm{OW}$ & -1.746821 & 3.158554 & 22.331116 \\
\hline HW & -2.222006 & 2.566844 & 22.982329 \\
\hline HW & -1.153117 & 3.791677 & 22.827777 \\
\hline OW & -3.168272 & 7.472166 & -4.651985 \\
\hline HW & -3.821112 & 7.900069 & -4.026925 \\
\hline $\mathrm{HW}$ & -3.524976 & 7.512401 & -5.585336 \\
\hline OW & -7.033018 & 5.661072 & 20.907104 \\
\hline HW & -6.988282 & 4.768628 & 21.356038 \\
\hline $\mathrm{HW}$ & -7.864158 & 6.137302 & 21.194179 \\
\hline OW & 1.389458 & -3.227477 & -20.219789 \\
\hline HW & 1.823174 & -4.128468 & -20.209463 \\
\hline HW & 2.092409 & -2.519988 & -20.292725 \\
\hline
\end{tabular}




\begin{tabular}{|c|c|c|c|}
\hline OW & -6.697646 & 7.299738 & 10.352816 \\
\hline HW & -6.787975 & 6.449691 & 9.833913 \\
\hline HW & -7.491701 & 7.882244 & 10.179145 \\
\hline OW & -9.129550 & -7.152772 & -8.850423 \\
\hline HW & -9.718307 & -6.702974 & -8.178823 \\
\hline HW & -9.387464 & -6.857711 & -9.770434 \\
\hline OW & 3.446804 & -17.065767 & 16.324817 \\
\hline HW & 3.639646 & -17.296389 & 17.278560 \\
\hline HW & 3.084398 & -17.870185 & 15.854100 \\
\hline OW & 3.119032 & 11.025400 & 2.126011 \\
\hline HW & 3.272023 & 11.281418 & 3.080500 \\
\hline HW & 3.986211 & 11.057946 & 1.629079 \\
\hline OW & -1.511907 & 16.565629 & 12.692808 \\
\hline HW & -0.937181 & 16.431624 & 11.885508 \\
\hline HW & -2.151125 & 15.801280 & 12.777491 \\
\hline OW & -6.832314 & -12.947611 & -0.817461 \\
\hline HW & -6.043581 & -13.036807 & -1.425691 \\
\hline HW & -6.692505 & -13.513795 & -0.005126 \\
\hline OW & 0.141924 & -14.405054 & 4.493358 \\
\hline HW & -0.664824 & -14.871350 & 4.856298 \\
\hline HW & 0.312746 & -14.714013 & 3.557750 \\
\hline OW & -3.622221 & -1.467275 & -20.698787 \\
\hline HW & -2.647322 & -1.689872 & -20.694101 \\
\hline HW & -3.740375 & -0.484388 & -20.557465 \\
\hline OW & -8.816110 & -11.180405 & -13.463387 \\
\hline HW & -9.236720 & -10.403935 & -12.994151 \\
\hline HW & -7.858082 & -10.971850 & -13.660077 \\
\hline OW & -7.631492 & 11.083026 & -0.917251 \\
\hline HW & -8.055337 & 10.181584 & -1.005325 \\
\hline HW & -7.796333 & 11.611645 & -1.749951 \\
\hline OW & 4.689045 & 5.483521 & -16.159760 \\
\hline HW & 3.796321 & 5.923584 & -16.256648 \\
\hline HW & 5.363138 & 5.971500 & -16.714264 \\
\hline OW & 7.937259 & -6.996395 & -6.706683 \\
\hline HW & 7.238545 & -6.648033 & -7.331537 \\
\hline HW & 7.610574 & -7.838403 & -6.277383 \\
\hline OW & 5.703076 & -7.148810 & 21.167583 \\
\hline HW & 4.925668 & -6.695101 & 21.603225 \\
\hline HW & 5.751538 & -8.098250 & 21.477770 \\
\hline OW & 6.893101 & 1.468358 & -6.431350 \\
\hline HW & 7.130090 & 0.527185 & -6.190454 \\
\hline HW & 7.690113 & 2.057373 & -6.297812 \\
\hline OW & 8.048657 & 6.868198 & -21.383096 \\
\hline HW & 7.260469 & 6.257467 & -21.307148 \\
\hline HW & 8.576562 & 6.631241 & -22.198674 \\
\hline OW & 8.632386 & 15.421438 & -17.659454 \\
\hline HW & 8.054985 & 14.620968 & -17.820253 \\
\hline HW & 8.663413 & 15.981726 & -18.487171 \\
\hline OW & 6.250762 & 14.085571 & -1.774769 \\
\hline HW & 5.660495 & 13.557931 & -2.385654 \\
\hline HW & 5.818763 & 14.159945 & -0.875966 \\
\hline OW & 4.827596 & -7.229674 & 18.370530 \\
\hline
\end{tabular}




\begin{tabular}{|c|c|c|c|}
\hline HW & 4.882812 & -7.456345 & 19.342935 \\
\hline HW & 4.515708 & -8.030244 & 17.858853 \\
\hline $\mathrm{OW}$ & -5.427735 & -0.564833 & 6.046805 \\
\hline HW & -5.158884 & 0.137122 & 5.387276 \\
\hline HW & -4.789121 & -0.561250 & 6.816324 \\
\hline OW & -9.093232 & 17.729758 & 20.858135 \\
\hline HW & -9.157472 & -17.152316 & 20.854058 \\
\hline HW & -9.984062 & 17.339355 & 21.090527 \\
\hline OW & 2.090201 & 15.526218 & -9.230294 \\
\hline HW & 2.807296 & 15.283615 & -9.883684 \\
\hline HW & 2.429513 & 15.394544 & -8.298881 \\
\hline OW & -7.876057 & -4.864891 & 18.050212 \\
\hline HW & -8.632763 & -5.454694 & 18.332221 \\
\hline HW & -7.657257 & -4.227301 & 18.788863 \\
\hline $\mathrm{OW}$ & -2.764378 & 16.926366 & 3.679803 \\
\hline HW & -3.474873 & 17.235816 & 3.047791 \\
\hline HW & -2.297062 & 17.719581 & 4.070220 \\
\hline OW & 3.385772 & 12.530060 & -1.017053 \\
\hline HW & 2.843344 & 12.207793 & -1.792885 \\
\hline HW & 3.225413 & 13.507758 & -0.881435 \\
\hline OW & -9.209644 & 17.291639 & -14.728819 \\
\hline HW & -8.822042 & 17.535120 & -15.617909 \\
\hline HW & -8.940180 & 16.358180 & -14.492084 \\
\hline OW & -1.733386 & 3.424739 & -7.172872 \\
\hline HW & -2.126615 & 4.066994 & -7.830808 \\
\hline HW & -0.870285 & 3.068000 & -7.530356 \\
\hline $\mathrm{OW}$ & 7.446544 & -5.960893 & 14.368135 \\
\hline HW & 7.175502 & -5.928107 & 15.330144 \\
\hline HW & 6.726579 & -5.555287 & 13.804988 \\
\hline OW & -3.352976 & 12.499698 & 15.534106 \\
\hline $\mathrm{HW}$ & -3.600251 & 13.468062 & 15.567650 \\
\hline HW & -3.984500 & 12.015202 & 14.928764 \\
\hline OW & 5.699345 & -9.801917 & 1.413754 \\
\hline $\mathrm{HW}$ & 6.245346 & -9.039058 & 1.760062 \\
\hline HW & 4.733873 & -9.541751 & 1.400373 \\
\hline OW & -9.619048 & -16.278176 & -1.884682 \\
\hline HW & -9.718774 & -16.782858 & -2.742208 \\
\hline HW & 10.253607 & -16.345364 & -1.357907 \\
\hline $\mathrm{OW}$ & -7.654996 & -10.976379 & 5.687068 \\
\hline HW & -8.251826 & -11.386379 & 4.997363 \\
\hline HW & -6.706926 & -11.011791 & 5.370984 \\
\hline OW & 8.110841 & 7.989705 & 15.219345 \\
\hline HW & 7.398217 & 8.316823 & 15.839958 \\
\hline HW & 8.963679 & 8.478094 & 15.404127 \\
\hline $\mathrm{OW}$ & 6.063998 & -5.442315 & -8.078509 \\
\hline HW & 6.552158 & -5.318935 & -8.942498 \\
\hline HW & 5.148689 & -5.801278 & -8.261141 \\
\hline $\mathrm{OW}$ & 1.922073 & 5.023698 & 5.319162 \\
\hline HW & 1.321510 & 4.396338 & 4.823437 \\
\hline HW & 2.597368 & 4.498983 & 5.837477 \\
\hline OW & 9.528702 & 2.124308 & 3.577005 \\
\hline HW & -10.223626 & 2.248506 & 3.796519 \\
\hline
\end{tabular}




\begin{tabular}{|c|c|c|c|}
\hline HW & 9.244971 & 2.819174 & 2.916203 \\
\hline OW & -0.144767 & -6.819528 & -9.055253 \\
\hline HW & -0.781013 & -6.789448 & -8.284353 \\
\hline HW & 0.781874 & -6.630628 & -8.730210 \\
\hline OW & 5.563719 & 6.746086 & 8.269071 \\
\hline HW & 5.098838 & 7.289024 & 7.569711 \\
\hline HW & 6.492791 & 6.535746 & 7.964798 \\
\hline OW & 4.496176 & -1.108618 & 12.126924 \\
\hline HW & 5.218884 & -1.670487 & 12.529412 \\
\hline HW & 4.030123 & -1.627966 & 11.410634 \\
\hline OW & 2.363011 & 14.910525 & -0.466816 \\
\hline HW & 1.874333 & 14.741774 & 0.389173 \\
\hline HW & 2.773387 & 15.822110 & -0.442213 \\
\hline OW & -6.346884 & 6.746065 & -8.928431 \\
\hline HW & -5.616143 & 6.578943 & -9.590314 \\
\hline HW & -6.373003 & 5.998375 & -8.264897 \\
\hline OW & 6.771246 & 7.398490 & -17.009118 \\
\hline HW & 7.749663 & 7.574393 & -16.900680 \\
\hline HW & 6.295454 & 8.259844 & -17.187140 \\
\hline OW & -4.583037 & -6.296222 & -10.154560 \\
\hline HW & -5.440371 & -6.789815 & -10.300659 \\
\hline HW & -4.381771 & -5.733260 & -10.956162 \\
\hline OW & 2.559103 & 16.837305 & -13.267283 \\
\hline HW & 2.798662 & 15.868167 & -13.325447 \\
\hline HW & 3.393973 & 17.387747 & -13.269684 \\
\hline OW & -9.436617 & -15.392562 & 21.544044 \\
\hline HW & -8.548355 & -14.951171 & 21.416907 \\
\hline HW & -9.811905 & -15.135923 & 22.434716 \\
\hline OW & 3.346181 & -13.124735 & -15.843446 \\
\hline HW & 3.060079 & -12.232984 & -15.492839 \\
\hline HW & 3.510825 & -13.058752 & -16.827590 \\
\hline OW & 0.076423 & -15.822577 & -10.332135 \\
\hline HW & -0.691481 & -15.568692 & -10.920239 \\
\hline HW & 0.934746 & -15.558720 & -10.772205 \\
\hline OW & 7.961422 & -6.284962 & -17.041785 \\
\hline HW & 8.384873 & -6.106830 & -16.153552 \\
\hline HW & 8.149302 & -5.521508 & -17.659716 \\
\hline OW & -4.141191 & -13.691160 & -3.065357 \\
\hline HW & -4.369170 & -14.661431 & -3.146591 \\
\hline HW & -3.178705 & -13.554175 & -3.299575 \\
\hline OW & -4.040454 & 3.978223 & 0.478295 \\
\hline HW & -4.064022 & 4.033620 & -0.519891 \\
\hline HW & -3.318427 & 3.346350 & 0.760099 \\
\hline OW & 3.445627 & 11.066744 & -8.956504 \\
\hline HW & 2.590869 & 11.585754 & -8.952235 \\
\hline HW & 3.434270 & 10.412048 & -9.712311 \\
\hline OW & 5.401732 & -12.135198 & 2.787957 \\
\hline HW & 4.479163 & -12.197966 & 3.168649 \\
\hline HW & 5.624062 & -11.176748 & 2.609235 \\
\hline OW & 9.783857 & -1.381705 & -21.526163 \\
\hline HW & 9.267022 & -1.714399 & -22.314958 \\
\hline HW & 9.522063 & -0.436582 & -21.330699 \\
\hline
\end{tabular}




\begin{tabular}{|c|c|c|c|}
\hline OW & 8.885011 & -3.786952 & 13.970637 \\
\hline HW & 8.635512 & -4.746900 & 14.098115 \\
\hline HW & 8.149044 & -3.312595 & 13.487585 \\
\hline OW & -1.463985 & 5.888799 & -11.833839 \\
\hline HW & -2.041834 & 6.231674 & -12.574465 \\
\hline HW & -0.617089 & 5.518860 & -12.215824 \\
\hline OW & -8.973149 & 11.491251 & -17.386966 \\
\hline HW & -9.299882 & 11.719632 & -18.304075 \\
\hline HW & -8.866186 & 10.500093 & -17.308455 \\
\hline OW & -5.453523 & 14.084068 & -6.220712 \\
\hline HW & -4.889622 & 13.320300 & -6.534838 \\
\hline HW & -5.427595 & 14.818532 & -6.898864 \\
\hline OW & -7.466099 & 2.335844 & 19.649699 \\
\hline HW & -8.151564 & 2.851749 & 19.135909 \\
\hline HW & -7.610164 & 2.474188 & 20.629549 \\
\hline OW & -9.116656 & -13.451326 & -2.246132 \\
\hline HW & -9.434712 & -14.313079 & -1.850884 \\
\hline HW & -8.417304 & -13.051048 & -1.653945 \\
\hline OW & 1.925172 & -10.956765 & 15.099246 \\
\hline HW & 2.031716 & -10.475394 & 15.969263 \\
\hline HW & 1.614174 & -11.891458 & 15.271376 \\
\hline $\mathrm{OW}$ & -8.066992 & -1.404950 & 16.709678 \\
\hline HW & -8.476275 & -0.881918 & 15.962065 \\
\hline HW & -7.550912 & -2.174876 & 16.334343 \\
\hline OW & 7.224601 & -14.442953 & -7.384405 \\
\hline HW & 8.071899 & -13.977153 & -7.129227 \\
\hline $\mathrm{HW}$ & 6.567194 & -13.773351 & -7.730021 \\
\hline OW & 2.176091 & 11.763133 & 11.683231 \\
\hline HW & 2.096961 & 10.851001 & 12.085416 \\
\hline HW & 1.265748 & 12.104121 & 11.448705 \\
\hline OW & -1.642701 & -8.113801 & 15.822740 \\
\hline HW & -0.741587 & -8.064362 & 15.391986 \\
\hline HW & -1.660556 & -7.520208 & 16.627307 \\
\hline OW & -3.419147 & -5.270175 & 21.652155 \\
\hline HW & -3.749135 & -5.754864 & 22.462206 \\
\hline HW & -2.553136 & -5.671217 & 21.353506 \\
\hline OW & 3.798760 & -0.333274 & -3.856996 \\
\hline HW & 3.456415 & 0.113757 & -3.030580 \\
\hline HW & 3.247847 & -1.145393 & -4.049239 \\
\hline OW & -8.013380 & 1.873696 & 10.299249 \\
\hline HW & -7.469061 & 1.684875 & 11.116600 \\
\hline HW & -8.688477 & 2.582234 & 10.504721 \\
\hline OW & 9.338460 & -9.387258 & -21.276541 \\
\hline HW & 9.312083 & -9.213691 & -22.261010 \\
\hline HW & 8.550695 & -9.945047 & -21.015203 \\
\hline OW & -5.639780 & -8.565568 & 10.861074 \\
\hline HW & -5.291020 & -9.461445 & 11.136338 \\
\hline HW & -6.628101 & -8.532554 & 11.009844 \\
\hline $\mathrm{OW}$ & -10.215274 & 2.639486 & -16.589344 \\
\hline HW & -9.525127 & 1.942335 & -16.395240 \\
\hline HW & -9.798005 & 3.546491 & -16.532525 \\
\hline OW & -0.257987 & 2.903062 & 8.515230 \\
\hline
\end{tabular}




\begin{tabular}{|c|c|c|c|}
\hline HW & 0.718819 & 3.039668 & 8.350342 \\
\hline HW & -0.785445 & 3.440390 & 7.857151 \\
\hline OW & 5.152044 & -12.366240 & -0.153609 \\
\hline HW & 4.322176 & -12.814600 & 0.178495 \\
\hline HW & 5.427769 & -11.654570 & 0.492533 \\
\hline OW & 0.154995 & -7.011953 & -11.652420 \\
\hline HW & 0.931728 & -7.606757 & -11.859528 \\
\hline HW & 0.116457 & -6.844946 & -10.667218 \\
\hline OW & 0.738936 & 11.583752 & -21.029835 \\
\hline HW & 0.750034 & 10.985134 & -21.830792 \\
\hline HW & 1.592565 & 12.103109 & -20.990020 \\
\hline OW & 3.243936 & 14.483035 & 11.412963 \\
\hline HW & 3.215317 & 14.386381 & 12.407869 \\
\hline HW & 3.371997 & 13.583975 & 10.994285 \\
\hline OW & 8.615311 & 9.351252 & -0.391289 \\
\hline HW & 9.516545 & 9.023975 & -0.675308 \\
\hline HW & 7.942236 & 9.128149 & -1.096410 \\
\hline OW & -3.966608 & -8.592115 & 14.299500 \\
\hline HW & -3.142290 & -8.507233 & 14.859228 \\
\hline HW & -4.235127 & -9.553864 & 14.245306 \\
\hline OW & -7.053343 & 15.092109 & -16.746606 \\
\hline HW & -6.531962 & 15.080235 & -15.893365 \\
\hline HW & -6.475889 & 14.765059 & -17.494660 \\
\hline OW & 9.827414 & -3.153499 & 16.639642 \\
\hline HW & 9.517294 & -3.308270 & 15.701627 \\
\hline HW & -10.228704 & -2.405765 & 16.652118 \\
\hline OW & -10.202065 & 11.580244 & 12.279370 \\
\hline HW & 10.214483 & 11.382231 & 13.211415 \\
\hline HW & 9.854988 & 11.211803 & 11.627640 \\
\hline OW & 6.817857 & -16.543385 & 2.477104 \\
\hline HW & 6.839531 & -15.580083 & 2.744646 \\
\hline HW & 6.513351 & -16.618832 & 1.527586 \\
\hline OW & -3.321344 & 0.182344 & -16.696145 \\
\hline HW & -2.646253 & 0.919894 & -16.679608 \\
\hline HW & -3.736909 & 0.090510 & -15.791230 \\
\hline OW & -0.875023 & 15.095349 & 7.820880 \\
\hline HW & -1.515618 & 15.052639 & 8.587571 \\
\hline HW & -0.145292 & 14.425617 & 7.958544 \\
\hline OW & 2.520912 & -13.848016 & 11.124609 \\
\hline HW & 3.078072 & -13.140305 & 11.559025 \\
\hline HW & 2.917143 & -14.746616 & 11.313075 \\
\hline OW & -8.781592 & -8.111392 & 10.484571 \\
\hline HW & -9.324022 & -8.853137 & 10.879013 \\
\hline HW & -9.024282 & -7.248654 & 10.928176 \\
\hline OW & -8.622389 & 8.485096 & -1.009447 \\
\hline HW & -8.900472 & 8.533310 & -1.968793 \\
\hline HW & -9.030975 & 7.677515 & -0.584159 \\
\hline OW & 5.805757 & -4.154802 & -17.087750 \\
\hline HW & 6.078117 & -5.094429 & -17.294927 \\
\hline HW & 5.575851 & -3.679467 & -17.936985 \\
\hline OW & 3.328465 & -1.312175 & -11.108610 \\
\hline HW & 3.139356 & -1.855210 & -11.926748 \\
\hline
\end{tabular}




\begin{tabular}{|c|c|c|c|}
\hline HW & 2.563392 & -1.394209 & -10.469912 \\
\hline OW & -7.600041 & -8.819606 & -16.144429 \\
\hline HW & -7.339807 & -9.430866 & -15.397007 \\
\hline HW & -7.664261 & -9.341626 & -16.994940 \\
\hline OW & -6.890019 & 2.421127 & -20.529150 \\
\hline HW & -7.624882 & 1.997621 & -19.999415 \\
\hline HW & -7.280065 & 2.905222 & -21.312423 \\
\hline OW & 4.793965 & 8.347878 & 6.223482 \\
\hline HW & 3.827598 & 8.604365 & 6.242167 \\
\hline HW & 4.945449 & 7.671612 & 5.502567 \\
\hline OW & -8.250020 & 8.867751 & -17.343507 \\
\hline HW & -7.258200 & 8.916271 & -17.461564 \\
\hline HW & -8.687045 & 8.759101 & -18.236371 \\
\hline OW & 4.131868 & 7.119243 & 19.509340 \\
\hline HW & 4.867498 & 6.498276 & 19.238694 \\
\hline HW & 4.392283 & 7.599438 & 20.346953 \\
\hline OW & 2.117136 & 10.979304 & -16.922319 \\
\hline HW & 2.462821 & 11.869236 & -17.219848 \\
\hline $\mathrm{HW}$ & 1.269291 & 10.771070 & -17.409963 \\
\hline OW & 9.594037 & -1.167694 & -0.189901 \\
\hline HW & 9.793616 & -1.718253 & 0.620688 \\
\hline HW & 8.605901 & -1.035440 & -0.267977 \\
\hline OW & -2.594711 & 0.239332 & 21.543786 \\
\hline HW & -1.623156 & 0.472905 & 21.582830 \\
\hline HW & -3.020606 & 0.436219 & 22.426877 \\
\hline OW & 8.370158 & -5.023972 & -0.187651 \\
\hline $\mathrm{HW}$ & 7.663806 & -4.388528 & -0.499544 \\
\hline HW & 8.478322 & -5.757576 & -0.858566 \\
\hline OW & -0.835616 & 12.261763 & -2.485509 \\
\hline HW & -1.688700 & 12.566024 & -2.909389 \\
\hline HW & -0.965377 & 12.179003 & -1.497424 \\
\hline OW & 6.537567 & -8.911625 & -5.179094 \\
\hline HW & 5.687927 & -8.399600 & -5.052833 \\
\hline HW & 6.358848 & -9.888368 & -5.060637 \\
\hline OW & -5.460715 & 1.721091 & 18.016952 \\
\hline HW & -6.063153 & 2.057697 & 18.740668 \\
\hline HW & -4.666202 & 1.272610 & 18.426358 \\
\hline OW & 5.026972 & -7.936460 & 11.989434 \\
\hline HW & 4.452810 & -7.807359 & 12.797933 \\
\hline HW & 4.458134 & -7.902006 & 11.167706 \\
\hline $\mathrm{OW}$ & 0.513187 & 15.273772 & 4.072053 \\
\hline HW & -0.187970 & 14.640623 & 4.399928 \\
\hline HW & 0.399696 & 16.159243 & 4.522674 \\
\hline OW & 1.061052 & 17.767264 & -21.490281 \\
\hline HW & 1.653150 & 17.341952 & -20.805788 \\
\hline HW & 1.562157 & 17.864676 & -22.350167 \\
\hline OW & 6.583121 & -0.425739 & 6.907066 \\
\hline HW & 7.113431 & -1.176975 & 6.514107 \\
\hline $\mathrm{HW}$ & 7.123610 & 0.029318 & 7.614734 \\
\hline OW & -7.677955 & -11.174508 & 16.122106 \\
\hline HW & -7.020042 & -10.421542 & 16.135963 \\
\hline HW & -7.441923 & -11.809879 & 15.386855 \\
\hline
\end{tabular}




\begin{tabular}{|c|c|c|c|}
\hline OW & 3.138791 & 16.917935 & 14.403417 \\
\hline HW & 2.375872 & 17.354156 & 13.926272 \\
\hline HW & 3.177793 & 15.950118 & 14.154805 \\
\hline OW & -5.664399 & -17.161965 & -7.607188 \\
\hline HW & -5.634041 & -16.266086 & -8.050447 \\
\hline HW & -6.490720 & -17.229361 & -7.048036 \\
\hline OW & 9.482623 & 4.751135 & 5.398396 \\
\hline HW & 9.051992 & 3.910647 & 5.727241 \\
\hline HW & 9.082318 & 5.008722 & 4.518962 \\
\hline OW & 9.039193 & -10.748215 & 19.054207 \\
\hline HW & 9.289939 & -10.908599 & 18.099533 \\
\hline HW & 9.521136 & -11.400283 & 19.639475 \\
\hline OW & 0.087573 & 14.394822 & 14.617151 \\
\hline HW & -0.368587 & 14.536052 & 15.495771 \\
\hline HW & 0.038309 & 15.235048 & 14.077157 \\
\hline OW & 10.053253 & -17.339500 & 8.473184 \\
\hline HW & -10.299679 & 17.857081 & 9.104215 \\
\hline HW & 9.731102 & -16.546862 & 8.990817 \\
\hline OW & -9.343140 & 5.183241 & 7.152814 \\
\hline HW & -9.946432 & 5.026216 & 6.370905 \\
\hline HW & -9.485129 & 4.464141 & 7.833060 \\
\hline $\mathrm{OW}$ & 5.715617 & -5.067411 & 7.939505 \\
\hline HW & 6.282934 & -5.669440 & 7.377622 \\
\hline HW & 4.927032 & -4.759884 & 7.407002 \\
\hline OW & -5.251737 & -11.195376 & -19.977885 \\
\hline HW & -5.171251 & -10.454893 & -20.645123 \\
\hline $\mathrm{HW}$ & -5.201288 & -12.075817 & -20.449348 \\
\hline OW & -8.846032 & 13.425505 & 13.510087 \\
\hline HW & -9.233961 & 12.953788 & 12.718257 \\
\hline HW & -7.925824 & 13.076811 & 13.687932 \\
\hline OW & -5.197438 & -3.492326 & 10.698222 \\
\hline HW & -4.544811 & -3.873012 & 11.353322 \\
\hline HW & -6.071737 & -3.970313 & 10.782661 \\
\hline OW & -5.037863 & 10.716257 & -21.415401 \\
\hline HW & -4.393830 & 10.269869 & -22.036657 \\
\hline HW & -4.543810 & 11.355323 & -20.825904 \\
\hline OW & 0.568979 & -7.932894 & 7.178371 \\
\hline HW & 0.137985 & -8.820823 & 7.339073 \\
\hline HW & 0.704496 & -7.465209 & 8.051816 \\
\hline OW & -5.567770 & 3.474893 & -18.372045 \\
\hline HW & -5.921568 & 3.077906 & -19.218939 \\
\hline HW & -5.806118 & 2.883507 & -17.601686 \\
\hline OW & -1.245103 & 13.813380 & 5.489398 \\
\hline HW & -1.103211 & 12.920776 & 5.917330 \\
\hline HW & -1.319854 & 14.514756 & 6.198259 \\
\hline OW & 7.943048 & 4.435111 & -7.930121 \\
\hline HW & 7.647373 & 4.687723 & -7.008837 \\
\hline HW & 7.489580 & 3.587277 & -8.204976 \\
\hline $\mathrm{OW}$ & -8.647459 & -5.598042 & -5.038090 \\
\hline HW & -7.847381 & -6.172478 & -5.211006 \\
\hline HW & -8.986721 & -5.768786 & -4.113024 \\
\hline OW & -5.710171 & 15.051841 & 20.319567 \\
\hline
\end{tabular}




\begin{tabular}{|c|c|c|c|}
\hline HW & -4.856953 & 14.673377 & 20.678434 \\
\hline HW & -5.866080 & 14.704317 & 19.394949 \\
\hline OW & 6.923792 & 5.127723 & -14.386595 \\
\hline HW & 7.035841 & 4.134082 & -14.375539 \\
\hline HW & 6.535515 & 5.409623 & -15.263963 \\
\hline OW & -5.525361 & 5.665608 & 15.775394 \\
\hline HW & -5.876084 & 6.575298 & 15.553006 \\
\hline HW & -5.640654 & 5.493936 & 16.753779 \\
\hline OW & 4.636260 & -13.558419 & -11.317426 \\
\hline HW & 4.625695 & -13.393238 & -12.303633 \\
\hline HW & 4.556548 & -12.687388 & -10.832708 \\
\hline OW & -1.932446 & -6.329957 & 4.902396 \\
\hline HW & -2.737666 & -6.898031 & 4.732359 \\
\hline HW & -1.907929 & -6.066631 & 5.866791 \\
\hline OW & -8.555920 & 2.613868 & 4.304197 \\
\hline HW & -8.251222 & 3.332603 & 4.929160 \\
\hline HW & -8.027431 & 2.663017 & 3.456680 \\
\hline OW & 2.682728 & 2.905824 & 12.832503 \\
\hline HW & 3.483708 & 2.851287 & 12.236301 \\
\hline HW & 2.538553 & 2.022561 & 13.278665 \\
\hline OW & -8.104614 & -15.952472 & -9.202749 \\
\hline HW & -8.662181 & -15.814391 & -10.021317 \\
\hline HW & -7.792523 & -15.066883 & -8.858754 \\
\hline OW & 6.238794 & 5.183494 & 19.209411 \\
\hline HW & 7.062698 & 5.666311 & 19.506178 \\
\hline HW & 6.258969 & 4.245690 & 19.555988 \\
\hline OW & 5.455236 & 3.297106 & -1.794851 \\
\hline HW & 6.272062 & 3.076442 & -1.261839 \\
\hline HW & 5.429951 & 4.280548 & -1.974301 \\
\hline OW & 9.226026 & 14.698774 & 18.005687 \\
\hline HW & 9.623099 & 15.615440 & 17.960333 \\
\hline HW & 9.019157 & 14.379021 & 17.081045 \\
\hline OW & -2.816228 & -2.328074 & -17.450564 \\
\hline HW & -3.020995 & -1.412317 & -17.104934 \\
\hline HW & -3.607510 & -2.680358 & -17.950333 \\
\hline OW & -8.028547 & -3.620498 & 7.533014 \\
\hline HW & -7.656545 & -3.773164 & 6.617423 \\
\hline HW & -8.113255 & -4.496062 & 8.008631 \\
\hline OW & 3.066406 & 1.050938 & -7.744461 \\
\hline HW & 3.521842 & 1.105125 & -8.633079 \\
\hline HW & 3.388885 & 1.794543 & -7.158748 \\
\hline OW & 4.393267 & -12.131048 & -3.251553 \\
\hline HW & 4.634185 & -12.208272 & -2.284084 \\
\hline HW & 5.081009 & -11.579255 & -3.723290 \\
\hline OW & 2.466203 & -4.040004 & 18.416216 \\
\hline HW & 2.248411 & -3.537865 & 19.253129 \\
\hline HW & 2.317498 & -3.448046 & 17.624084 \\
\hline OW & -5.092424 & 2.237203 & -4.176119 \\
\hline HW & -4.636595 & 2.914944 & -3.599155 \\
\hline HW & -4.933557 & 2.457798 & -5.138459 \\
\hline OW & -5.722350 & -5.590742 & -19.182329 \\
\hline HW & -5.272965 & -5.036152 & -19.882674 \\
\hline
\end{tabular}




$\begin{array}{lrrc}\text { HW } & -6.311827 & -6.268705 & -19.621514 \\ \text { OW } & 2.976591 & -5.853611 & 21.597023 \\ \text { HW } & 3.305663 & -5.109214 & 22.178043 \\ \text { HW } & 2.278303 & -5.504796 & 20.971945 \\ \text { OW } & 3.058673 & -6.312771 & 1.398811 \\ \text { HW } & 2.839416 & -7.266448 & 1.604786 \\ \text { HW } & 4.051548 & -6.199567 & 1.361625 \\ \text { OW } & -7.105343 & -0.385622 & 12.191945 \\ \text { HW } & -7.698916 & -0.931456 & 11.600559 \\ \text { HW } & -7.448387 & -0.418992 & 13.130671 \\ \text { OW } & 4.652661 & 1.490676 & 11.338721 \\ \text { HW } & 4.512120 & 0.600940 & 11.773023 \\ \text { HW } & 5.380712 & 1.983097 & 11.815651 \\ \text { OW } & 4.502191 & -10.508075 & -13.648834 \\ \text { HW } & 3.692315 & -10.407705 & -14.226784 \\ \text { HW } & 5.027097 & -9.656986 & -13.659859 \\ \text { OW } & 4.583377 & 3.206302 & -14.539157 \\ \text { HW } & 4.687255 & 3.517485 & -13.594501 \\ \text { HW } & 4.482707 & 3.997997 & -15.141722 \\ \text { OW } & 6.890870 & -14.759495 & 15.158291 \\ \text { HW } & 7.029974 & -15.254255 & 16.016116 \\ \text { HW } & 6.528895 & -13.848102 & 15.354089\end{array}$

